"This is the accepted manuscript of an article published by Elsevier in http://dx.doi.org/10.1016/j.bmc.2016.06.045

(C) 2016. This manuscript version is made available under the CC-BY-NC-ND 4.0 license http://creativecommons.org/licenses/by-nc-nd/4.0/

\& Medicinal Chemistry

Elsevier Editorial System(tm) for Bioorganic

Manuscript Draft

Manuscript Number:

Title: Design, synthesis and biological evaluation of $\mathrm{N}$-methyl-N-[(1,2,3triazol-4-yl)alkyl]propargylamines as novel monoamine oxidase B inhibitors

Article Type: SI: Jorgensen Prize

Keywords: Click-chemistry; monoamine oxidase B; Alzheimer's disease; irreversible inhibition

Corresponding Author: Prof. F. J. Luque,

Corresponding Author's Institution:

First Author: Ornella Di Pietro

Order of Authors: Ornella Di Pietro; Nelson Alencar; Gerard Esteban; Elisabet Viayna; Natalia Szalaj; Javier Vázquez; Jordi Juárez-Jiménez; Irene Sola; Montse Solé; Mercedes Unzeta; Diego Muñoz-Torrero; F. J. Luque

Abstract: Different azides and alkynes have been coupled via Cu-catalyzed 1,3-dipolar Huisgen cycloaddition to afford a novel family of N1- and C5substituted 1,2,3-triazole derivatives that feature the propargylamine group typical of irreversible MAO-B inhibitors at the C4-side chain of the triazole ring. All the synthesized compounds were evaluated against human MAO-A and MAO-B. Structure-activity relationships and molecular modelling were utilized to gain insight into the structural and chemical features that enhance the binding affinity and selectivity between the two enzyme isoforms. Several lead compounds, in terms of potency (submicromolar to low micromolar range), MAO-B selective recognition, and brain permeability, were identified. One of these leads (MAO-B IC50 of $3.54 \mu \mathrm{M}$, selectivity MAO-B/MAO-A index of 0.04) was further subjected to reversibility and time-dependence inhibition studies, which disclosed a slow and irreversible inhibition of human MAO-B. Overall, the results support the suitability of the 4-triazolylalkyl propargylamine scaffold for exploring the design of multipotent anti-Alzheimer compounds endowed with irreversible MAO-B inhibitory activity. 


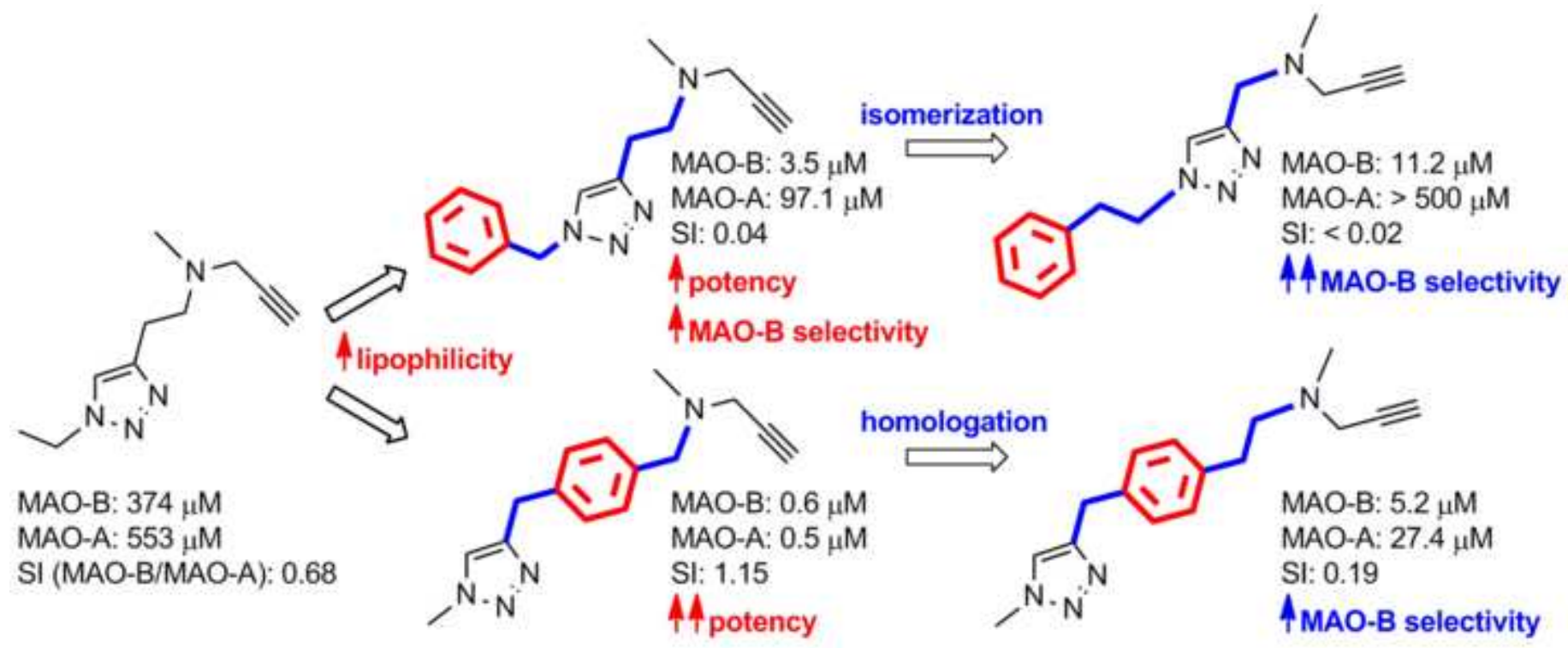




\section{Design, synthesis and biological evaluation of} $N$-methyl- $N$-[(1,2,3-triazol-4yl)alkyl]propargylamines as novel monoamine oxidase B inhibitors

Ornella Di Pietro, ${ }^{[\mathrm{a}]}$ Nelson Alencar, ${ }^{[\mathrm{b}]}$ Gerard Esteban, ${ }^{[\mathrm{c}]}$ Elisabet Viayna, ${ }^{[\mathrm{a}]}$ Natalia Szałaj, ${ }^{[\mathrm{a}]}$ Javier Vázquez, ${ }^{[\mathrm{b}]}$ Jordi Juárez-Jiménez, ${ }^{[\mathrm{b}]}$ Irene Sola, ${ }^{[\mathrm{a}]}$ Belén Pérez, ${ }^{[\mathrm{d}]}$ Montse Solé, ${ }^{[\mathrm{c}]}$ Mercedes Unzeta, ${ }^{[\mathrm{c}]}$ Diego Muñoz-Torrero, ${ }^{*[\mathrm{a}]}$ F. Javier Luque ${ }^{*[\mathrm{~b}]}$

[a] Laboratori de Química Farmacèutica (Unitat Associada al CSIC), Facultat de Farmàcia and Institut de Biomedicina (IBUB), Universitat de Barcelona, Av. Joan XXIII 27-31, E-08028 Barcelona, Spain

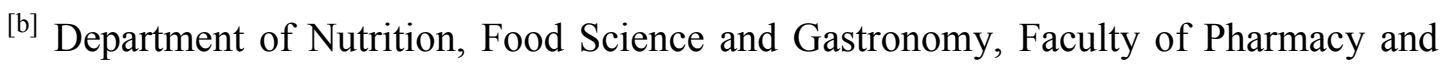
Institute of Biomedicine (IBUB), University of Barcelona, Av. Prat de la Riba 171, E08921 Santa Coloma de Gramenet, Spain

${ }^{[c]}$ Departament de Bioquímica i Biologia Molecular, Facultat de Medicina and Institut de Neurociències, Universitat Autònoma de Barcelona, E-08193 Bellaterra, Spain

${ }^{\text {[d] }}$ Departament de Farmacologia, Terapéutica i Toxicologia, Universitat Autònoma de Barcelona, E-08193 Bellaterra, Spain

*E-mail: dmunoztorrero@ub.edu; fjluque@ub.edu 


\begin{abstract}
Different azides and alkynes have been coupled via $\mathrm{Cu}$-catalyzed 1,3-dipolar Huisgen cycloaddition to afford a novel family of $N_{1^{-}}$and $C_{5}$-substituted 1,2,3-triazole derivatives that feature the propargylamine group typical of irreversible MAO-B inhibitors at the $C 4$-side chain of the triazole ring. All the synthesized compounds were evaluated against human MAO-A and MAO-B. Structure-activity relationships and molecular modelling were utilized to gain insight into the structural and chemical features that enhance the binding affinity and selectivity between the two enzyme isoforms. Several lead compounds, in terms of potency (submicromolar to low micromolar range), MAO-B selective recognition, and brain permeability, were identified. One of these leads (MAO-B $\mathrm{IC}_{50}$ of $3.54 \mu \mathrm{M}$, selectivity MAO-B/MAO-A index of 0.036) was further subjected to reversibility and time-dependence inhibition studies, which disclosed a slow and irreversible inhibition of human MAO-B. Overall, the results support the suitability of the 4-triazolylalkyl propargylamine scaffold for exploring the design of multipotent anti-Alzheimer compounds endowed with irreversible MAO-B inhibitory activity.
\end{abstract}

\title{
Keywords
}

Click-chemistry; monoamine oxidase B; Alzheimer's disease; irreversible inhibition 


\section{Introduction}

Alzheimer's disease (AD) is one of the most relevant age-related neurodegenerative disorders currently representing the fourth leading cause of death and afflicting over 46.8 million people worldwide. ${ }^{1,2}$ Its clinical manifestation is mainly reflected in a progressive loss of memory and cognitive functions, often in association with behavioral disturbances and depression. ${ }^{3}$ Its complex and multifaceted etiopathology, which involves massive loss of cholinergic neurons, ${ }^{4}$ oxidative stress, ${ }^{5-7}$ metal dyshomeostasis, ${ }^{8}$ excitotoxicity, ${ }^{9}$ neurofibrillary tangles and $\beta$-amyloid aggregate deposition, ${ }^{10,11}$ has precluded so far the discovery of effective disease-modifying drugs. Currently approved drugs, namely, three acetylcholinesterase (AChE) inhibitors (rivastigmine, galantamine, and donepezil), ${ }^{12-14}$ and an $N$-methyl- $D$-aspartate receptor antagonist (memantine), ${ }^{15}$ primarily exert palliative effects. On the other hand, the failure in clinical trials of a number of drug candidates designed against targets mainly involved in $\beta$-amyloid biology has shifted drug discovery efforts toward the compounds hitting less explored biological targets alone or in combination with other key targets, i.e. the so-called multi-target-directed ligands (MTDLs). ${ }^{16-18}$

In this context, monoamine oxidase (MAO, E.C.1.4.3.4) has emerged as a promising target because of the neuroprotective properties exerted by their inhibitors. ${ }^{2,19-21}$ MAO is a flavin adenine dinucleotide (FAD)-containing enzyme that catalyzes the degradation of biogenic and xenobiotic amines. Two isoforms, namely MAO-A and MAO-B, have been characterized by their amino acid sequence, tissue distribution, substrate specificity and inhibitor sensitivity. ${ }^{22-24}$ MAO-A, preferentially degrading serotonin, adrenaline and noradrenaline, is irreversibly inhibited by clorgyline, whereas MAO-B, specifically responsible for the oxidative deamination of phenylethylamine and benzylamine, is irreversibly inhibited by $(R)-(-)$-deprenyl (selegiline) (Figure 1). These trends reflect structural differences in the binding sites as revealed by highresolution X-ray structures. ${ }^{25-29}$ In particular, a key structural feature in shaping the substrate cavity is the replacement of the pair Phe208/Ile335 in MAO-A by Ile199/Tyr326 in MAO-B, leading to the distinction between "substrate" and "entrance" sites in MAO-B. The replacement of Ile180/Asn181 and Val210 in MAO-A by Leu17/Cys172 and Thr201 in MAO-B are additional differences in the binding sites, which may modulate the selective inhibition by certain MAO inhibitors. ${ }^{30}$ 
<smiles>C#CCN(C)CCCCOc1ccc(Cl)cc1Cl</smiles>

Clorgyline, 1

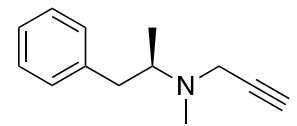

R-(-)-Deprenyl, 2

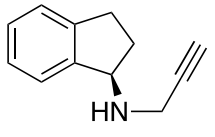

Rasagiline, 3<smiles>C#CCNCc1cc(OC(=O)N(C)CC)ccc1C</smiles>

Ladostigil, 4

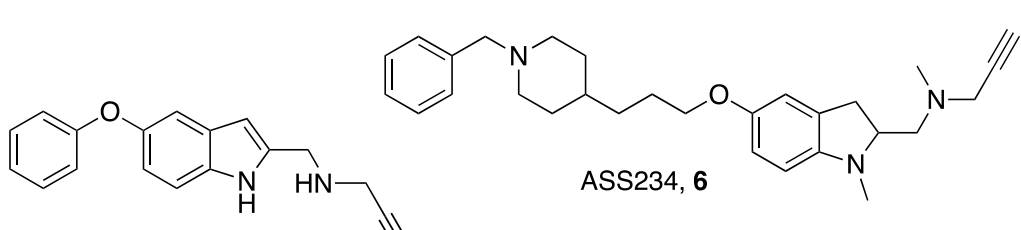

PF9601N, 5

Figure 1. Chemical structures of MAO inhibitors.

The neuroprotection exerted by MAO inhibitors may stem not only from the increased amine neurotransmission, but also from preventing the formation of neurotoxic species, which may lead to neuronal damage, ${ }^{31,32}$ and from the anti-apoptotic properties of the propargylamine group present in some MAO inhibitors. ${ }^{33,34}$ Interestingly, the levels of MAO-B increase with age and its activity is elevated in AD patients, which results in increased brain levels of neurotoxic free radicals. ${ }^{20}$ In this context, the development of MAO-B-inhibitor-based MTDLs emerges as a promising strategy for the design of neuroprotective agents with potential disease-modifying activity towards $\mathrm{AD}$ and other neurodegenerative disorders, such as Parkinson disease. ${ }^{35,36}$

Most efforts have been addressed toward the design of MTDLs targeting AChE and/or butyrylcholinesterase (BuChE) and MAO. A successful example is ladostigil (Figure 1), a dual inhibitor of MAO-B and AChE that combines the carbamate moiety of the AChE inhibitor rivastigmine with the indolamine moiety of the selective MAO-B inhibitor rasagiline, and shows neuroprotective and anti-apoptotic activities. ${ }^{37,38}$ A novel series of $\mathrm{MAO} / \mathrm{ChE}$ inhibitors that combine the $N$-benzylpiperidine moiety of the AChE inhibitor donepezil with the indolyl propargylamine of the potent MAO-B inhibitor PF9601N has been reported. ${ }^{39}$ Within this series, the most promising MDTL compound (ASS234, 6 in Figure 1) also showed anti-aggregating activity on $\beta$-amyloid, antioxidant and antiapoptotic behaviour, and crossed easily the blood-brain barrier (BBB). ${ }^{40}$ Other strategies have relied on the hybridization of coumarins with either $\mathrm{N}$-benzyl- $\mathrm{N}$ - 
alkyloxy groups ${ }^{41}$ or tacrine, ${ }^{42}$ leading to multipotent inhibitors of both MAO and ChEs, or alternatively have pursued the development of MTDLs targeting both MAO inhibition and additional activities, such as metal chelation. ${ }^{43,44}$ Also, natural products endowed with MAO and ChEs inhibitory activities have been recently reported. ${ }^{45}$

Although the development of MAO-inhibitor-based MTDLs is very attractive, it is challenged by the need to keep a good balance among potencies against multiple targets and optimal ADME-T properties. ${ }^{46-50}$ Furthermore, the success of this strategy depends on the suitability of an efficient synthetic approach, which should afford the fusion or linkage of chemical scaffolds while minimizing drastic alterations in the activity against the multiple targets. In this context, the $\mathrm{Cu}(\mathrm{I})$-catalyzed azide-alkyne cycloaddition (CuAAC) enables the synthesis of a virtually unlimited source of ligands containing the 1,4-disubstituted 1,2,3-triazole core. ${ }^{51-54}$ The CuAAC reaction between libraries of azides and alkynes featuring different pharmacophoric moieties has been used for the synthesis of triazole-linked hybrid compounds as MTDLs. ${ }^{55-65}$ Azide-alkyne cycloaddition reactions have also been used for the synthesis of high-affinity multisite enzyme inhibitors inside the biological target, in the absence of copper catalysis, i.e. the so-called in situ click chemistry. ${ }^{66-70}$ In these compounds the triazole ring is used not only as a non-hydrolyzable, non-oxidizable, and non-reducible robust linker between the pharmacophoric moieties, but also provides favourable physicochemical properties and potential interactions with the biological target. ${ }^{71}$

In this context, as the first step of a program directed to the synthesis of MAO-Binhibitor-based anti-Alzheimer MTDLs, here we have explored the CuAAC-mediated synthesis of a series of 1,2,3-triazole derivatives, featuring the propargylamine group of typical irreversible MAO-B inhibitors in the side chain at position 4, while the overall hydrophobicity has been modulated through different lipophilic substituents at positions 1 and 5 (Scheme 1). To determine the therapeutic potential of the target compounds and their usefulness as the MAO-B pharmacophoric moiety of novel families of MTDLs, we have assessed the in vitro inhibitory activity of the novel compounds against human MAO-B and MAO-A. Molecular modelling studies have been performed to rationalize the differences in inhibitory potency and selectivity between the MAO isoforms, and the mechanism of action of these compounds has been studied by reversibility and timedependence inhibition studies. Finally, the brain penetration of the novel compounds has been examined through the widely used parallel artificial membrane permeability assay (PAMPA-BBB). 


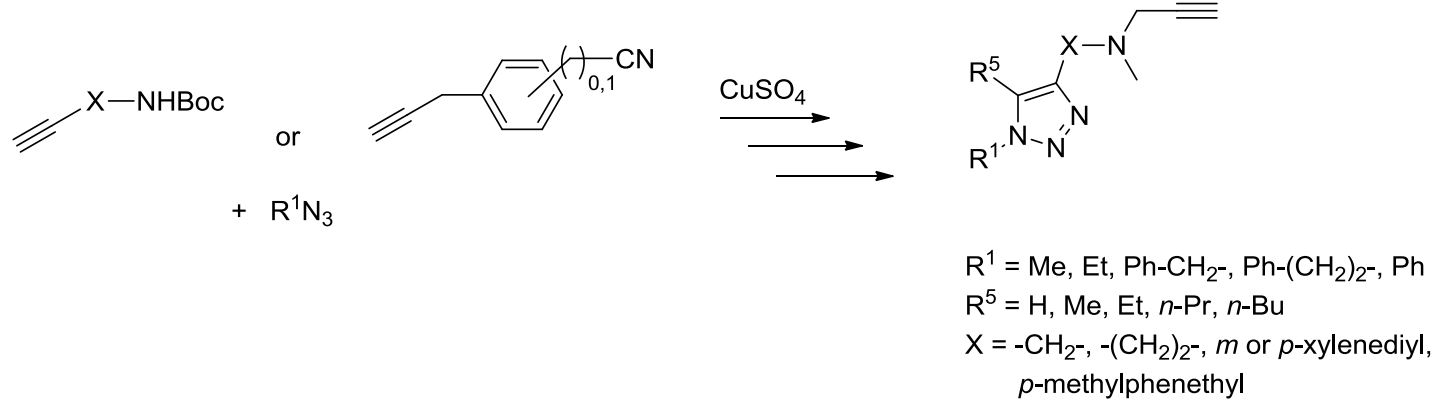

Scheme 1. General structure of the target $N$-methyl- $N-[(1,2,3$-triazol-4yl)alkyl]propargylamines.

\section{Results and Discussion}

2.1. Design and synthesis of the target $N$-methyl-N-[(1,2,3-triazol-4yl)alkyl]propargylamines

We initially planned the synthesis of the 1-ethyltriazolylmethyl and 1-ethyltriazolylethyl propargylamines 31 and $\mathbf{3 2}$ (Scheme 2), unsubstituted at position 5 of the triazole ring, and their 5-methyl-substituted analogues $\mathbf{5 1}$ and $\mathbf{5 2}$, as the early simple prototypes to validate the suitability of the CuAAC-assisted synthetic methodology to deliver 1,4disubstituted and 1,4,5-trisubstituted 1,2,3-triazole scaffolds featuring the propargylamine group of irreversible MAO inhibitors. These compounds are quite polar $(\log P \text { of } 0.43,0.84,0.65 \text {, and } 1.06 \text {, respectively) })^{72}$ due to the presence of the triazole ring. However, the substrate cavity of MAO is highly hydrophobic, and accordingly MAO inhibitors are usually more lipophilic molecules ( $\log P$ in the range 2-4 for compounds depicted in Figure 1). Thus, to counterbalance the high polarity conferred by the triazole ring, we envisaged a second generation of derivatives that featured more lipophilic benzyl or phenethyl substituents at position 1 (i.e. compounds $\mathbf{3 3}$ and $\mathbf{3 4}$, respectively, Scheme 2) or at position 5 of the triazole ring (i.e. the 1-methyl-5-butylsubstituted compound 54). We also planned the synthesis of derivatives of the 1-benzylsubstituted compound $\mathbf{3 3}$ bearing different alkyl groups at position 5. However, the moderate acidity of the $\alpha$-nitrogen benzylic protons of the substituent at position 1 of the triazole ring interfered with the alkylation of the position 5 (see below). To avoid these synthetic issues, we envisaged the synthesis of compounds 55, 56, and 57 (Scheme 2), bearing a phenyl group instead of a benzyl group at position 1 of the 
triazole ring and ethyl, propyl, or butyl substituents at position 5. Finally, we also explored the isomerization of the benzene ring of compound $\mathbf{3 3}$ from the substituent at position 1 of the triazole ring to the side chain at position 4 , i.e. between the triazole ring and the propargylamine moiety (compounds $\mathbf{8 2}$ and $\mathbf{8 3}$, Scheme 3 ).

After evaluation of MAO-A and MAO-B inhibitory activity of the first and second generation compounds, $\mathbf{3 3}$ emerged as the most selective MAO-B inhibitor, with a onedigit micromolar potency against MAO-B, 27-fold higher than against MAO-A, whereas compound $\mathbf{8 3}$ turned out to be the most potent inhibitor of both MAO isoforms, with submicromolar potencies but essentially without any selectivity (see below). In light of these results and on the basis of molecular modelling studies, we inferred that a total number of three methylenes might afford the optimal length between the terminal aromatic ring and the propargylamine nitrogen atom to confer selectivity towards MAO-B. To check this hypothesis, as a third generation we planned the synthesis of compound 36 (Scheme 2), an isomeric derivative of 33, and compound 84 (Scheme 3), with a total number of three methylenes from the terminal aromatic ring (phenyl in $\mathbf{3 6}$, triazole in $\mathbf{8 4}$ ) to the propargylamine nitrogen atom. For comparison purposes, we also envisaged the synthesis of compound $\mathbf{3 5}$ (Scheme 2), the shorter homologue of $\mathbf{3 3}$ with a total number of two methylenes in the tether.

The synthesis of the target compounds was undertaken through synthetic sequences involving as the key step a CuAAC reaction ${ }^{73-76}$ between alkyl or phenyl azides and alkynes bearing an amino or a cyano group to enable the subsequent installation of the propargylamine moiety (Scheme 1). The synthesis of the 5-unsubstituted triazolylmethyl and triazolylethyl propargylamines 31-36 was carried out through a four-step synthetic sequence, starting from the copper-catalyzed Huisgen 1,3-dipolar cycloaddition of the known $N$-Boc-protected amines $\mathbf{7}$ and $\mathbf{8}$ with ethyl, benzyl, and phenethyl azide, generated in situ by reaction of $\mathrm{NaN}_{3}$ with the corresponding alkyl halide ${ }^{77}$ which afforded the 1-substituted triazole derivatives 9-16 in moderate to good yields (49-78\%, Scheme 2). Subsequent methylation of the $N$-Boc-protected aliphatic nitrogen atom of compounds $\mathbf{9 - 1 6}$ with $\mathrm{MeI}$ in anhydrous THF, in the presence of $\mathrm{NaH}$, followed by acidic deprotection of the resulting compounds 17-24 afforded the secondary amines 25-30 in general in excellent yields. The final propargylation of amines 25-30 on reaction with propargyl bromide in acetone in the presence of $\mathrm{Cs}_{2} \mathrm{CO}_{3}$ led to propargylamines 31-36 in 22-89\% yield (Scheme 2). Thus, the target compounds 
31-36 were obtained in 5-58\% overall yield without the need of any column chromatography purification, with the sole exception of the last step for $\mathbf{3 1}, \mathbf{3 5}$ and $\mathbf{3 6}$.
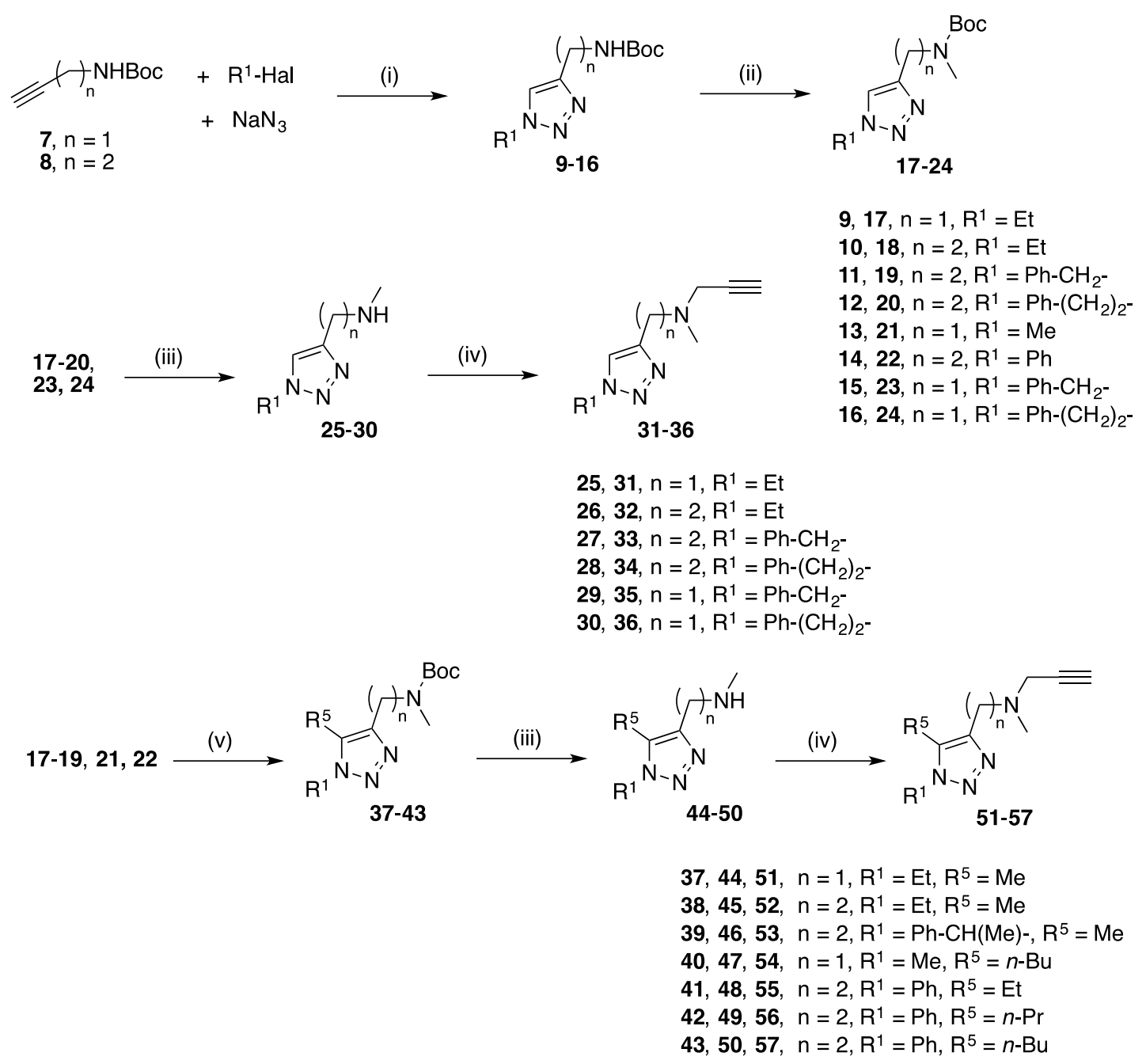

Scheme 2. Reagents and conditions: (i) $\mathrm{CuSO}_{4} \cdot 5 \mathrm{H}_{2} \mathrm{O}$, ascorbic acid, $\mathrm{Na}_{2} \mathrm{CO}_{3}$, $\mathrm{H}_{2} \mathrm{O} / \mathrm{DMF}$, rt, overnight, 49-78\% yield; (ii) MeI, NaH, THF, rt, 1.5-15 h, 81-97\% yield; (iii) $\mathrm{H}_{3} \mathrm{PO}_{4}, \mathrm{CH}_{2} \mathrm{Cl}_{2}$, rt, 1.5-4 h, 38-98\% (25-30), 67-100\% (44-50); (iv) propargyl bromide, $\mathrm{Cs}_{2} \mathrm{CO}_{3}$, acetone, $\mathrm{rt}$ for $2-15 \mathrm{~h}$, or $0{ }^{\circ} \mathrm{C}$ for $2-2.5 \mathrm{~h}$, or $0{ }^{\circ} \mathrm{C}$ for 30 min and then rt for $3 \mathrm{~h}, 22-89 \%$ (31-36), 53-95\% (51-57); (v) $\mathrm{R}^{5}$-Hal, $n$-BuLi, THF, $-78^{\circ} \mathrm{C}, 1 \mathrm{~h}$, then $-78^{\circ} \mathrm{C} \rightarrow \mathrm{rt}$, and $\mathrm{rt}, 2 \mathrm{~h}, 43-98 \%$.

For the synthesis of the 5-alkyl-substituted triazolylmethyl and triazolylethyl propargylamines $\mathbf{5 1}, \mathbf{5 2}$, and $\mathbf{5 4}$, apart from the intermediates $\mathbf{1 7}$ and $\mathbf{1 8}$, the triazole 
derivative 21 was also prepared in good yield using the same CuAAC- $N$-methylation protocol (Scheme 2). Alkylation of compounds 17, 18, and 21 with the appropriate alkyl halide using $n$-BuLi as the base afforded the corresponding 5-alkylated compounds $\mathbf{3 7}$, 38, and 40, respectively, in excellent yields (90-97\%). However, when we subjected the 1-benzyl-substituted intermediate $\mathbf{1 9}$ to the same alkylation reaction conditions with methyl iodide as the alkylating agent, we did not obtain the expected 1-benzyl-5methyl-substituted derivative but the 1-( $\alpha$-methylbenzyl)-5-methyl-substituted derivative 39 in $79 \%$ yield, resulting from the double alkylation at the position 5 of the triazole ring and at the moderately acidic $\alpha$-nitrogen benzylic position of the substituent at position 1 (Scheme 2). Treatment of intermediate 19 with ethyl bromide or $n$-butyl bromide as the alkylating agents and $n$-BuLi or the more hindered $t$-BuLi as the base yielded inseparable mixtures of 5-monoalkylated and $\alpha, 5$-dialkylated products. To avoid double alkylation, we prepared the 1-phenyl-substituted intermediate 22, which was alkylated with ethyl bromide, $n$-propyl bromide, and $n$-butyl bromide using $n$-BuLi as the base, to afford the expected 5-alkyl-substituted derivatives $\mathbf{4 1}, \mathbf{4 2}$, and $\mathbf{4 3}$ in $98 \%$, $96 \%$, and $43 \%$ yield, respectively (Scheme 2). The acidic $N$-Boc deprotection of 5 alkyl-substituted compounds $\mathbf{3 7 - 4 3}$ afforded the secondary amines $\mathbf{4 4 - 5 0}$ in good yields (67\% to quantitative yield). Propargylation of amines $\mathbf{4 4 - 5 0}$ had to be performed by monitoring the reaction course at different reaction temperatures and times to avoid the formation of dipropargylated products. For example, reaction of amine $\mathbf{4 4}$ with propargyl bromide in the presence of $\mathrm{Cs}_{2} \mathrm{CO}_{3}$ at $\mathrm{rt}$ overnight yielded the dipropargylated derivative as the major reaction product ( $92 \%$ yield), whereas on reaction at $0{ }^{\circ} \mathrm{C}$ for $2 \mathrm{~h}$ only the desired monopropargylated product, 51, was obtained, in $62 \%$ yield. Thus, by selecting the most appropriate reaction temperature and time, the target 5-alkylsubstituted triazolylmethyl and triazolylethyl propargylamines 51-57 were synthesized (19-44\% overall yield over the five-step sequence).

The synthesis of the triazolyl- $m$-xylyl and triazolyl- $p$-xylyl propargylamines $\mathbf{8 2}$ and $\mathbf{8 3}$ and the triazolyl-p-methylphenethyl propargylamine $\mathbf{8 4}$ was carried out through the seven-eight-step sequences depicted in Scheme 3. First, the alkynes 64-66, necessary for the CuAAC, were prepared by an initial Negishi coupling ${ }^{77}$ between benzyl bromides 58-60 and trimethylsilylacetylene in the presence of $n$-BuLi and $\mathrm{ZnBr}_{2}$, under $\operatorname{Pd}(0)$ catalysis, followed by desilylation of the protected alkyne derivatives 61-63 with AgOTf in a mixture of $\mathrm{CH}_{2} \mathrm{Cl}_{2} / \mathrm{MeOH} / \mathrm{H}_{2} \mathrm{O},{ }^{78}$ and silica gel column chromatography purification. The CuAAC of methyl azide to alkynes 64-66 afforded the corresponding 
triazole derivatives 67-69 in excellent yield (89-95\%). $\mathrm{LiAlH}_{4}$ reduction of the nitrile group of 67 and 68 afforded the corresponding amines 70 and 71 in $49 \%$ and $80 \%$ yield, respectively. However, the same reaction conditions failed to provide the amine derived from nitrile $\mathbf{6 9}$, which could be efficiently reduced to amine $\mathbf{7 2}$ by hydrogenation using Raney-Ni as the catalyst. $N$-Boc protection of the primary amino group of 70-72 afforded the intermediates 73-75. The $N$-Boc-protected amines $\mathbf{7 3}$ and $\mathbf{7 4}$ were converted into the target propargylamines $\mathbf{8 2}$ and $\mathbf{8 3}$ in good yields through the standard $\mathrm{N}$-methylation-acidic deprotection-propargylation protocol, without the need of any additional column chromatography purification (Scheme 3). Because different attempts to methylate the $N$-Boc-protected amine 75 were fruitless, this compound was alternatively converted into the target propargylamine $\mathbf{8 4}$ by direct conversion into the secondary amine 81 upon $\mathrm{LiAlH}_{4}$ reduction followed by progargylation (Scheme 3).

All the compounds to be subjected to biological evaluation were transformed into the corresponding hydrochloride salts and were chemically characterized through IR, ${ }^{1} \mathrm{H}$ and ${ }^{13} \mathrm{C}$ NMR spectra, and HRMS.
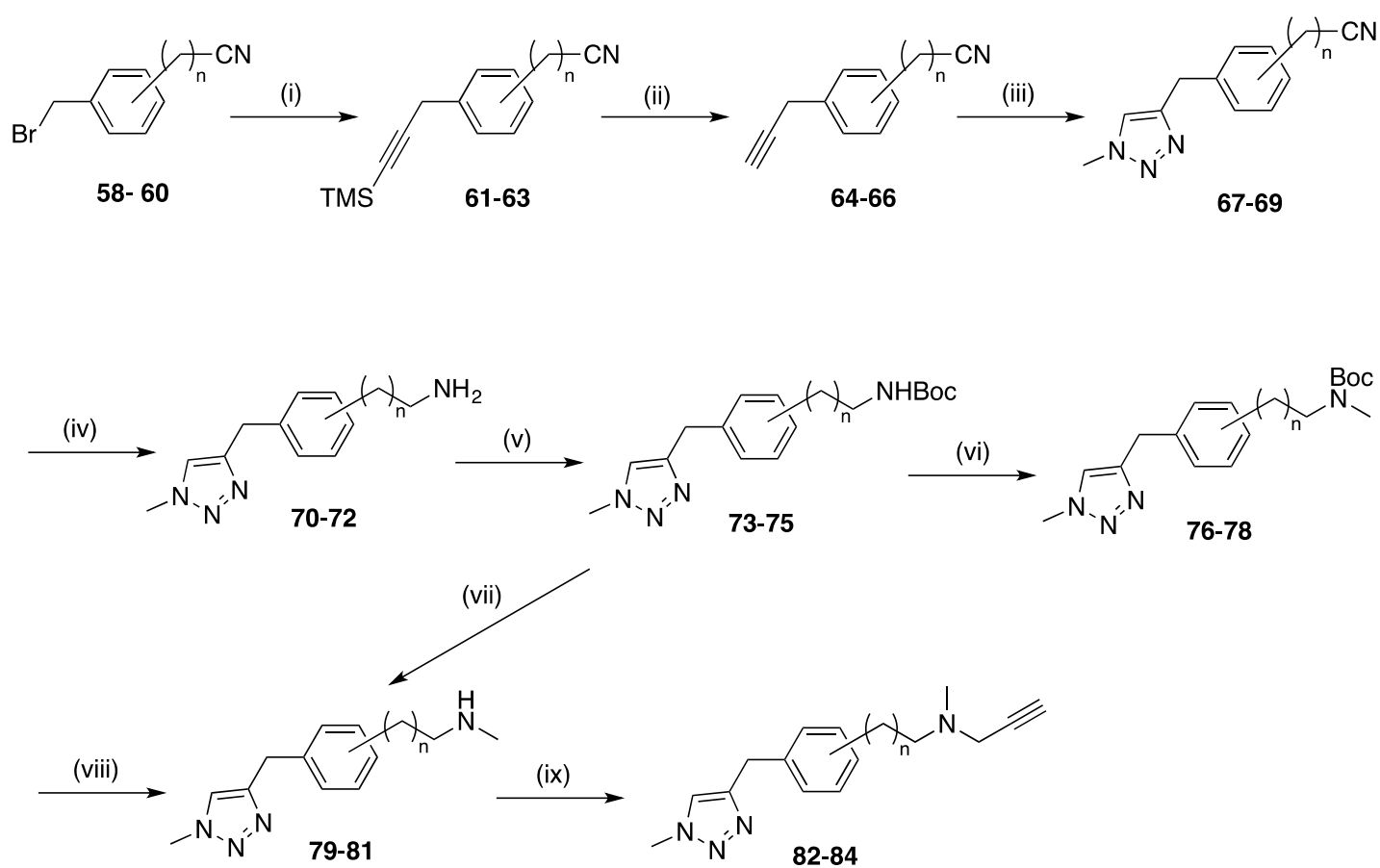

Series meta: $\mathrm{n}=0,58,61,64,67,70,73,76,79,82$ 
Scheme 3. Reagents and conditions: (i) trimethylsilylacetylene, THF, $n$-BuLi, $-78{ }^{\circ} \mathrm{C}$, 30 min, then, $\mathrm{ZnBr}_{2}$, THF, $-78{ }^{\circ} \mathrm{C} \rightarrow 0{ }^{\circ} \mathrm{C}$, then, 58, 59, or 60, $\mathrm{Cl}_{2} \mathrm{Pd}_{(}\left(\mathrm{PPh}_{3}\right)_{2}, \mathrm{rt}$, overnight, 44-83\% yield; (ii) AgOTf, $\mathrm{CH}_{2} \mathrm{Cl}_{2} / \mathrm{MeOH} / \mathrm{H}_{2} \mathrm{O}$, rt, overnight, 63-77\% yield; (iii) $\mathrm{CuSO}_{4} \cdot 5 \mathrm{H}_{2} \mathrm{O}$, ascorbic acid, $\mathrm{Na}_{2} \mathrm{CO}_{3}, \mathrm{H}_{2} \mathrm{O} / \mathrm{DMF}$, rt, overnight, 89-95\% yield; (iv) $\mathrm{LiAlH}_{4}$, THF, reflux, 2 h: 70 (49\%), 71 (80\%), or $\mathrm{H}_{2}$, Raney-Ni, $7 \mathrm{~N} \mathrm{NH}_{3}$ in MeOH: 72 (quantitative); (v) (Boc) ${ }_{2} \mathrm{O}, \mathrm{THF}, \mathrm{rt}, 3 \mathrm{~h}$, quantitative yield; (vi) $\mathrm{MeI}, \mathrm{NaH}, \mathrm{THF}, 60^{\circ} \mathrm{C}$, 4 h: 76 (84\%), 77 (80\%); (vii) $\mathrm{LiAlH}_{4}$, THF, $0{ }^{\circ} \mathrm{C}$, then 75, reflux, $72 \mathrm{h:} 81$ (44\% yield); (viii) $\mathrm{H}_{3} \mathrm{PO}_{4}, \mathrm{CH}_{2} \mathrm{Cl}_{2}, \mathrm{rt}, 1.5-3 \mathrm{~h}$ : 79 (quantitative), 80 (96\%); (ix) propargyl bromide, $\mathrm{Cs}_{2} \mathrm{CO}_{3}$, acetone, $0{ }^{\circ} \mathrm{C}$ for $3.5 \mathrm{~h}$ or $0{ }^{\circ} \mathrm{C}$ for $30 \mathrm{~min}$ and then $\mathrm{rt}$ for $3 \mathrm{~h}, 31-65 \%$ yield.

\subsection{MAO inhibitory activity of the target compounds}

The novel $N$-methyl- $N$-[(1,2,3-triazol-4-yl)alkyl]propargylamines 31-36, 51-57, and 82-84 were evaluated in vitro against human recombinant MAO-A and MAO-B ( $h r \mathrm{MAO}-\mathrm{A}$ and $h r \mathrm{MAO}-\mathrm{B}$ ). Selegiline and clorgyline were also used as reference compounds. The corresponding $\mathrm{IC}_{50}$ values and selectivity indices (SI; defined as the ratio $\left.\mathrm{IC}_{50}(\mathrm{MAO}-\mathrm{B}) / \mathrm{IC}_{50}(\mathrm{MAO}-\mathrm{A})\right)$ are shown in Table 1 . The novel compounds turned out to be in most cases moderate to weak MAO inhibitors, with 2-3-digit micromolar $\mathrm{IC}_{50}$ values, with the exceptions of compounds $33,55,82$ and 83 , which exhibited submicromolar or low micromolar potencies against one or both MAO isoforms.

The first generation compounds 31, 32, 51, and $\mathbf{5 2}$ were weak inhibitors and showed no selectivity. These trends were not unexpected due to the large polarity of the triazole ring and the small size of these fragment-like compounds. Accordingly, several strategies were explored to modulate both the molecular size and hydrophobicity of the triazole derivatives. The first approach involved the substitution of the ethyl group at position 1 of the triazole ring of the initial prototypes by benzyl and phenethyl groups, which led to increased inhibitory potencies. Thus, the 1-phenethyl-derivative $\mathbf{3 4}$ was 2and 10-fold more potent MAO-A inhibitor than the 1-benzyl- and 1-ethyl-analogues $\mathbf{3 3}$ and 32, respectively. In contrast, for MAO-B inhibition, the optimal substitution pattern involved the presence of a 1-benzyl group, with compound 33 being 49- and 106-fold more potent than the 1-phenethyl- and 1-ethyl-analogues 34 and 32, respectively. 
Table 1. MAO-A and MAO-B inhibitory activities, selectivity indices, and BBB permeability.

\begin{tabular}{|c|c|c|c|c|}
\hline Compound & $\begin{array}{l}h r \mathrm{MAO}-\mathrm{A}^{\mathrm{a}} \\
\mathrm{IC}_{50}(\mu \mathrm{M})\end{array}$ & $\begin{array}{l}h r \mathrm{MAO}-\mathrm{B}^{\mathrm{a}} \\
\mathrm{IC}_{50}(\mu \mathrm{M})\end{array}$ & $\mathrm{SI}^{\mathrm{b}}$ & $P e\left(10^{-6} \mathrm{~cm} \mathrm{~s}^{-1}\right)^{\mathrm{c}}$ \\
\hline \multicolumn{5}{|c|}{ 1st generation } \\
\hline 31 & $134.7 \pm 21.8$ & $248.6 \pm 94.5$ & 1.8 & $\mathrm{nd}^{\mathrm{d}}$ \\
\hline 32 & $553.5 \pm 83.6$ & $373.9 \pm 119$ & 0.7 & $\mathrm{nd}^{\mathrm{d}}$ \\
\hline 51 & $267.4 \pm 30.8$ & $104.7 \pm 27.1$ & 0.4 & $\mathrm{nd}^{\mathrm{d}}$ \\
\hline 52 & $91.2 \pm 13.6$ & $237.6 \pm 51.8$ & 2.6 & $\mathrm{nd}^{\mathrm{d}}$ \\
\hline \multicolumn{5}{|c|}{ 2nd generation } \\
\hline 33 & $97.1 \pm 30.1$ & $3.54 \pm 0.44$ & 0.036 & $15.6 \pm 1.5(\mathrm{CNS}+)$ \\
\hline 34 & $56.1 \pm 8.7$ & $172.8 \pm 47.8$ & 3.1 & $14.8 \pm 0.9(\mathrm{CNS}+)$ \\
\hline 53 & $47.0 \pm 6.9$ & $134.2 \pm 25.5$ & 2.9 & $20.0 \pm 0.7(\mathrm{CNS}+)$ \\
\hline 54 & $<300$ & $94.73 \pm 16.3$ & $<0.32$ & $29.9 \pm 0.8(\mathrm{CNS}+)$ \\
\hline 55 & $7.5 \pm 1.1$ & $213.4 \pm 54.0$ & 28.5 & $17.0 \pm 1.9(\mathrm{CNS}+)$ \\
\hline 56 & $26.1 \pm 4.0$ & $156.8 \pm 31.9$ & 6.0 & $17.2 \pm 0.6(\mathrm{CNS}+)$ \\
\hline 57 & $17.3 \pm 4.5$ & $155.5 \pm 25.0$ & 9.0 & $21.4 \pm 1.3(\mathrm{CNS}+)$ \\
\hline 82 & $2.12 \pm 0.32$ & $64.96 \pm 15.4$ & 30.6 & $15.6 \pm 1.5(\mathrm{CNS}+)$ \\
\hline 83 & $0.53 \pm 0.06$ & $0.60 \pm 0.08$ & 1.15 & $14.8 \pm 0.9(\mathrm{CNS}+)$ \\
\hline \multicolumn{5}{|c|}{ 3rd generation } \\
\hline 35 & $>500$ & $129 \pm 42.6$ & $<0.26$ & $2.3 \pm 0.7(\mathrm{CNS}+/-)$ \\
\hline 36 & $>500$ & $11.2 \pm 2.1$ & $<0.022$ & $0.6 \pm 0.1$ (CNS-) \\
\hline 84 & $27.4 \pm 0.1$ & $5.22 \pm 0.49$ & 0.19 & $4.5 \pm 0.5(\mathrm{CNS}+/-)$ \\
\hline $\begin{array}{l}\text { Selegiline } \\
\text { Clorgyline }\end{array}$ & $\begin{array}{c}2 \pm 0.1 \\
0.020 \pm 0.003\end{array}$ & $\begin{array}{c}0.018 \pm 0.003 \\
\mathrm{nd}^{\mathrm{d}}\end{array}$ & 0.009 & \\
\hline
\end{tabular}

a In vitro inhibitory activities against $h r$-MAO-A and $h r$-MAO-B. Experiments performed with Amplex Red fluorimetric method in 96-well plates. Data are the mean of duplicate experiments \pm SEM.

${ }^{\mathrm{b}} \mathrm{SI}=\mathrm{IC}_{50}$ (MAO-B) / IC 50 (MAO-A).

${ }^{\mathrm{c}}$ Permeability values from the PAMPA-BBB assay. Values are expressed as the mean \pm SD of three independent experiments. 
${ }^{\mathrm{d}}$ Not determined.

This latter finding suggests that the size of compound $\mathbf{3 3}$ is better suited to fill the binding cavity in MAO-B, as confirmed by the results obtained from restrained docking calculations. Thus, following the strategy used in our previous studies, ${ }^{39}$ the propargylamine nitrogen atom was imposed to overly the position of the corresponding atom in the X-ray structures of covalently-bound selegiline and rasagiline (PDB entries 2BYB and 2BK4) ${ }^{28,79}$ By imposing this restraint, we may explore the arrangement of the compound after covalent linkage of the propargylamine unit to the FAD cofactor. Figure 2 shows that $\mathbf{3 3}$ superposes well with the structures of the irreversible inhibitor $\mathbf{6}$ (PDB entry 4CRT) ${ }^{80}$ and the reversible ligand 1,4-diphenyl-2-butene (PDB entry 1OJ9) ${ }^{26}$ which spans the substrate binding cavity. In particular, it is worth noting that the benzene ring of $\mathbf{3 3}$ matches well the position filled by the benzene unit in 1,4diphenyl-2-butene. In turn, docking of the phenethyl derivative $\mathbf{3 4}$ made it necessary to adopt a folded conformation, reflecting some degree of internal strain due to clashes within the cavity (data not shown).

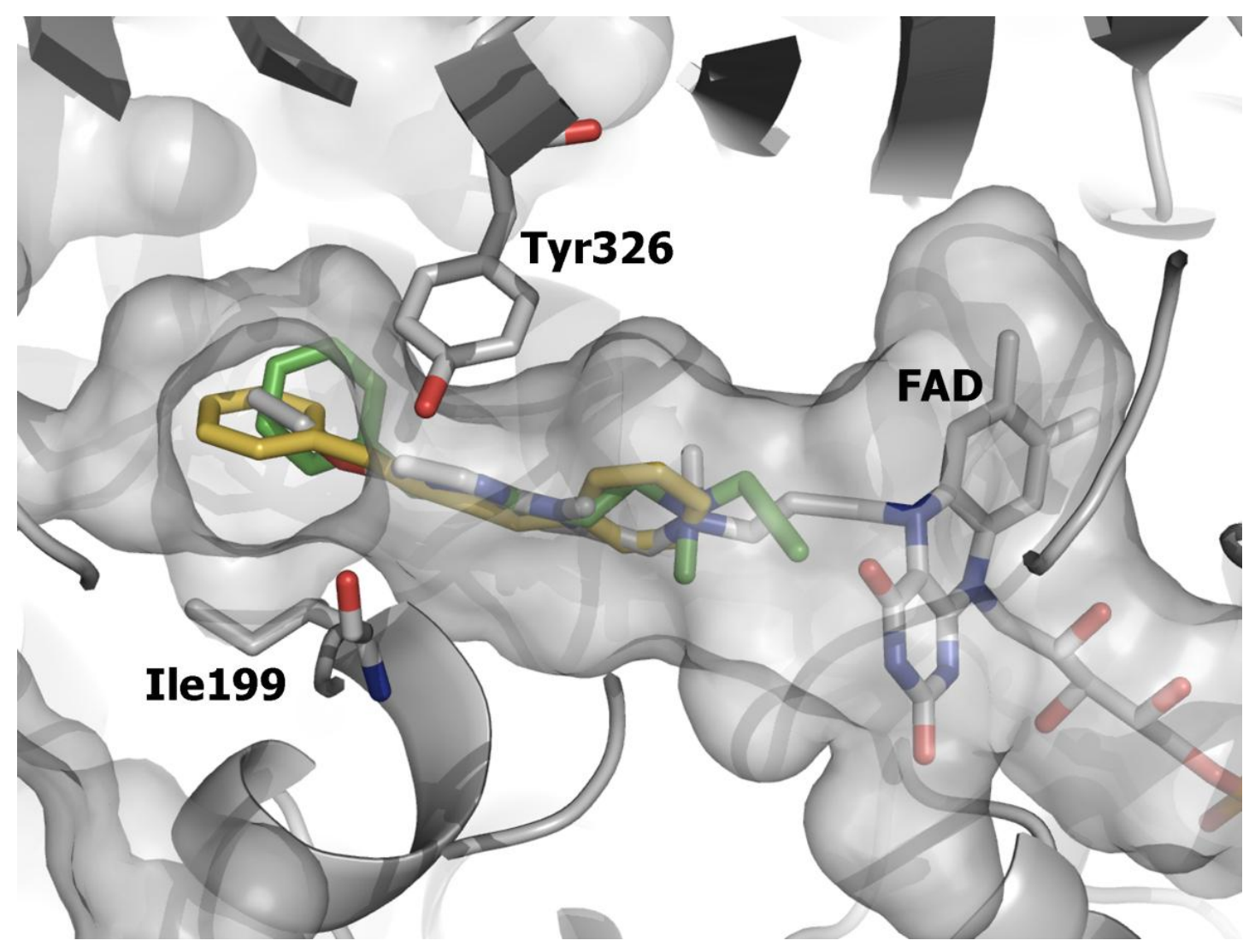


Figure 2. Representation of the docked pose of compound $\mathbf{3 3}$ (shown as green-coloured sticks) in the binding cavity of MAO-B (shown as gray cartoon). The surface of the binding cavity is depicted as a gray isocontour. The structures of inhibitor 6 (PDB entry 4CRT; covalently-bound to FAD, also shown as gray-coloured sticks) and the reversible ligand 1,4-diphenyl-2-butene (PDB entry 1OJ9) are shown as white-colored and yellow sticks, respectively.

An alternative strategy explored to modulate the hydrophobicity distribution of triazole derivatives relied on the alkylation of position 5 of the triazole ring (compounds 53-57). However, alkylation did not seem to have a clear effect on MAO inhibitory potency, although this chemical modification was generally beneficial for MAO-A selectivity for compounds bearing large substituents at position $1(\mathrm{Ph}-\mathrm{CH}(\mathrm{Me})-$ or $\mathrm{Ph})$, with selectivity indices in the range 2.9-28.5 (compounds 53, 55-57 in Table 1).

Finally, we also envisaged the isomerization of the benzene ring of compound $\mathbf{3 3}$ from the substituent at position 1 of the triazole ring to the side chain at position 4 . This approach led to increased MAO-A inhibitory potencies, with compounds $\mathbf{8 2}$ and $\mathbf{8 3}$ being 46- and 183-fold more potent than 33, and also to increased MAO-B inhibitory activity in the case of $\mathbf{8 3}$ (6-fold more potent than $\mathbf{3 3}$ ), bearing a para-disubstituted benzene ring in the side chain at position 4 . Docking calculations of compound $\mathbf{8 3}$ also led to a binding pose that filled the binding cavity of MAO-B (Figure 3), while docking of the derivative with a meta-disubstituted benzene (compound 82) revealed steric clashes in the substrate binding cavity (data not shown), in agreement with the 100-fold ratio in the $\mathrm{IC}_{50}$ values determined for these compounds against $h r \mathrm{MAO}-\mathrm{B}$ (Table 1). In agreement with the hypothesis that a total number of three methylenes separating the terminal aromatic ring and the propargylamine nitrogen atom afforded the optimal length to confer selectivity towards MAO-B, third generation compounds $\mathbf{3 6}$ and $\mathbf{8 4}$ turned out to be selective for MAO-B versus MAO-A inhibition, with $\mathrm{IC}_{50}$ values for MAO-B inhibition in the low micromolar range. Compound $\mathbf{3 6}$ was found to be more selective MAO-B inhibitor than its shorter homologue 35, and even more selective than its isomer $\mathbf{3 3}$ with the same total number of methylenes in the tether (27-fold more potent for MAO-B than for MAO-A inhibition; Table 1). Also, in contrast with compounds 82 and 83 , with the inverse selectivity or with no selectivity, compound 84 
was found to be 5-fold more potent for MAO-B than for MAO-A inhibition, albeit with a 9-fold lower potency than its shorter homologue $\mathbf{8 3}$.

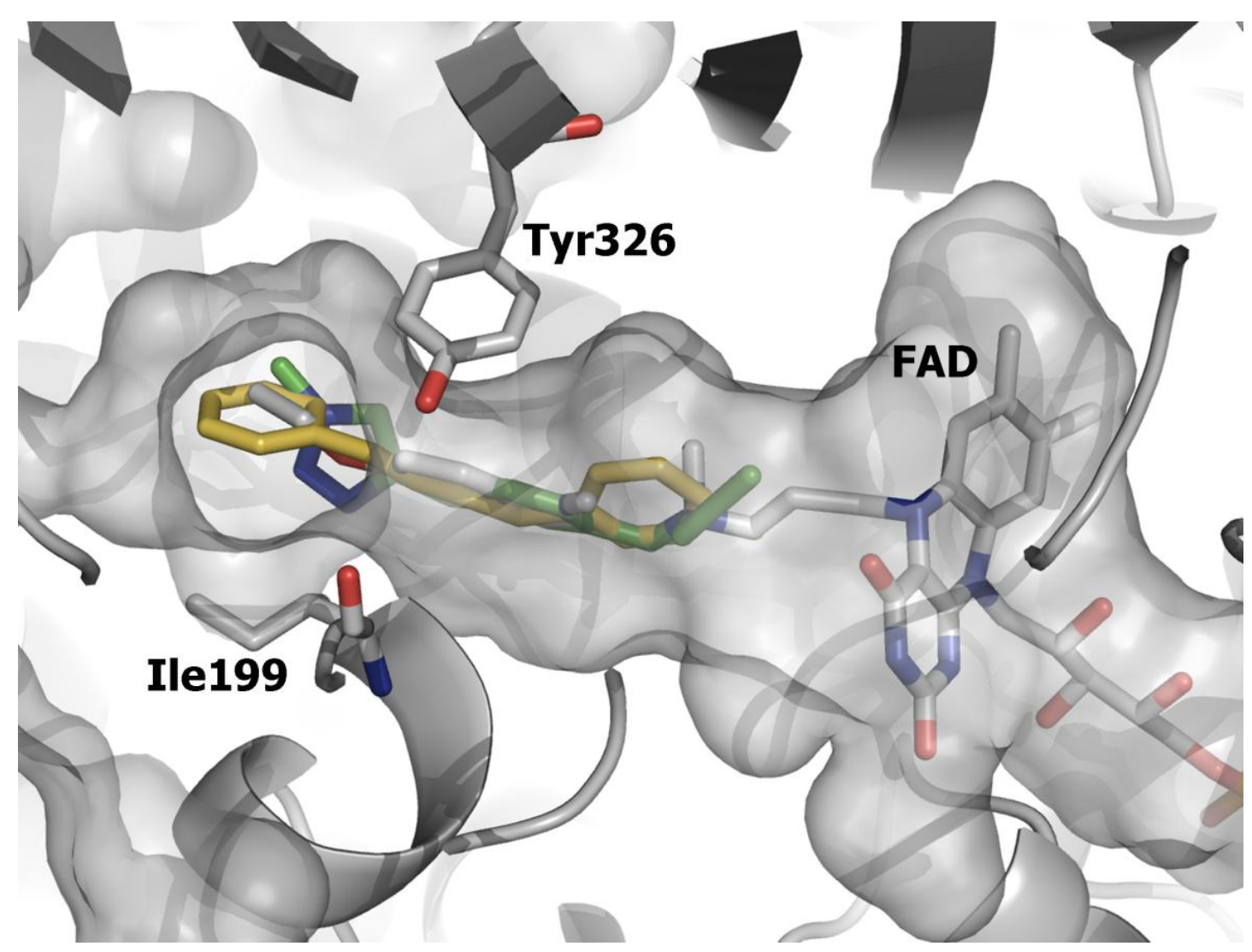

Figure 3. Representation of the docked pose of compound $\mathbf{8 3}$ (shown as green-coloured sticks) in the binding cavity of MAO-B (shown as gray cartoon). The surface of the binding cavity is depicted as a gray isocontour. The structures of inhibitor $\mathbf{6}$ (PDB entry 4CRT; covalently-bound to FAD, also shown as gray-coloured sticks) and the reversible ligand 1,4-diphenyl-2-butene (PDB entry 1OJ9) are shown as white-colored and yellow sticks, respectively.

Overall, compound $\mathbf{8 3}$ emerges as the most potent MAO inhibitor of the series, with submicromolar potencies against both MAO-A and MAO-B, and hence, essentially without selectivity, whereas compounds $\mathbf{3 3}, \mathbf{3 6}$, and $\mathbf{8 4}$, with a total number of three methylene groups in the tethers that connect their phenyl and triazole rings with the 
propargylamine nitrogen atom, emerge as low micromolar selective MAO-B inhibitors of interest as novel leads for AD drug discovery.

\subsection{Reversibility and time-dependent inhibition studies}

The triazole derivative 33, one the most promising compounds of the series by virtue of its one-digit micromolar inhibitory potency and MAO-B selectivity, was subjected to further studies to gain insight into the type of inhibition of MAO-B. First, we investigated whether the propargylamino group present in $\mathbf{3 3}$ led to the irreversible inhibition of the enzyme. As shown in Figure 4, the reversibility assays confirmed that 33 irreversibly inhibited MAO-B, since the inhibition was not reverted after various cycles of consecutive centrifugations and washings with buffer. Furthermore, this finding is in agreement with the time-dependent inhibition of MAO-B resulting from incubation with 33, as shown in Figure 4. Thus, after pre-incubation of $h r \mathrm{MAO}-\mathrm{B}$ with a $10 \mu \mathrm{M}$ concentration of $\mathbf{3 3}$ for different times ranging from 5 to $360 \mathrm{~min}$, the enzymatic activity showed a slow time-dependent inhibition of MAO-B. These findings mimic the behavior reported for the MTDL inhibitor $6,{ }^{39}$ and for the structurally related MAO-B inhibitor PFN9601. ${ }^{81}$ As previously noted for $6{ }^{39}$ these results suggest that the proper accommodation of $\mathbf{3 3}$ in a conformation suitable for covalent attachment to the FAD cofactor may require an a priori conformational rearrangement for the structural adaptation to the peculiar shape of the MAO-B binding pocket.
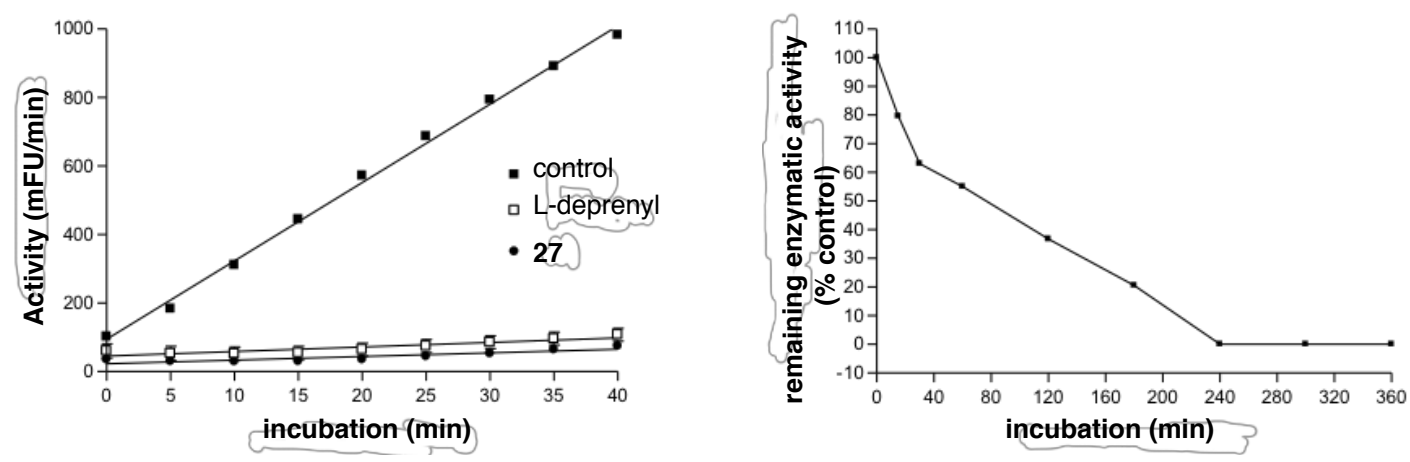

Figure 4. Plot of the enzymatic activity ( $\mathrm{mFU} / \mathrm{min})$ versus pre-incubation time (min) (left) and plot of the remaining enzymatic activity (as \% of control sample) versus preincubation time (right) in the study of reversibility of $h r \mathrm{MAO}-\mathrm{B}$ inhibition by $\mathbf{3 3}$. 


\subsection{Blood-brain barrier permeation assay}

Because an essential feature of anti-neurodegenerative drug candidates is the ability to cross the BBB, all the novel compounds were subjected to the well-established PAMPA-BBB assay, as an in vitro model of passive permeation. ${ }^{82}$ The in vitro permeability $(\mathrm{Pe})$ of the novel compounds through a lipid extract of porcine brain was determined using a mixture of phosphate-buffered saline (PBS)/EtOH 70:30. Assay validation was carried out by comparison of the experimental and reported permeability values of 14 commercial drugs (see Table S1 in Supplementary Material), which provided a good linear correlation: $P e(\exp )=1.003 P e($ lit $)-0.783\left(R^{2}=0.93\right)$. Using this equation and the limits established by Di et al. for BBB permeation, ${ }^{82}$ the following ranges of permeability were established: $P e\left(10^{-6} \mathrm{~cm} \mathrm{~s}^{-1}\right)>5.18$ for compounds with high BBB permeation $(\mathrm{CNS}+)$; $P e\left(10^{-6} \mathrm{~cm} \mathrm{~s}^{-1}\right)<2.06$ for compounds with low BBB permeation (CNS-); and $5.18>P e\left(10^{-6} \mathrm{~cm} \mathrm{~s}^{-1}\right)>2.06$ for compounds with uncertain BBB permeation $(\mathrm{CNS} \pm)$. As shown in Table 1, the tested compounds had generally $P e$ values well above the threshold for high BBB permeation, so that they were predicted to be able to cross the $\mathrm{BBB}$ and reach their biological target in the CNS, although unexpectedly the third generation compounds showed lower permeabilities than the rest of compounds, maybe as a consequence of solubility issues

\section{Conclusion}

The results reported in this study support the feasibility of the CuAAC-mediated synthesis of 1,2,3-triazole derivatives featuring the propargylamine group typical of irreversible MAO-A and MAO-B inhibitors, leading to suitable templates for the design of MAO-B inhibitor-based MTDLs. The synthetic procedures of the 1,4-disubstituted or 1,4,5-trisubstituted 1,2,3-triazole scaffolds involve 4-6-step sequences from the $\mathrm{CuAAC}$ of alkyl or phenyl azides with an alkyne bearing an $N$-Boc-protected primary amino or a cyano group as precursors of the propargylamine warhead. Nevertheless, introduction of lipophilic substitutents were necessary to counterbalance the high polarity conferred by the triazole ring and to modulate the inhibitory activity against the two MAO isoforms. The triazolyl- $p$-xylyl propargylamine $\mathbf{8 3}$ has been found to be a moderately potent nonselective MAO inhibitor, with submicromolar potencies against both human MAO-A and MAO-B. On the other hand, the isomeric 1benzyltriazolylethyl propargylamine $\mathbf{3 3}$ and 1-phenethyltriazolylmethyl propargylamine 
36, and the triazolyl-p-methylphenethyl propargylamine $\mathbf{8 4}$ have emerged as moderately potent and selective MAO-B inhibitor with low micromolar potencies and large selectivity indices, with an appropriate hydrophilic-lipophilic balance (LogP values of 2.1, 1.9, and 2.4, respectively, i.e. in the range of classical MAO inhibitors) and relatively low molecular weight (254-268). Further work will be focused on refining the MAO-B inhibitory potency of these scaffolds by enabling the linkage to a second pharmacophoric moiety to derive novel MTDLs, as well as to develop small-molecule probes for MAO-B imaging in models of neurodegenerative diseases. ${ }^{83}$

\section{Experimental part}

\subsection{Chemistry. General methods}

Melting points were determined in open capillary tubes with a MFB 595010M Gallenkamp melting point apparatus. $400 \mathrm{MHz}{ }^{1} \mathrm{H} / 100.6 \mathrm{MHz}{ }^{13} \mathrm{C}$ NMR spectra were recorded on a Varian Mercury 400 spectrometer at the Centres Científics i Tecnològics of the University of Barcelona (CCiTUB). The chemical shifts are reported in $\mathrm{ppm}(\delta$ scale) relative to solvent signals $\left(\mathrm{CD}_{3} \mathrm{OD}\right.$ at 3.31 and $49.0 \mathrm{ppm}$ in the ${ }^{1} \mathrm{H}$ and ${ }^{13} \mathrm{C}$ NMR spectra, respectively; $\mathrm{CDCl}_{3}$ at 7.26 and $77.16 \mathrm{ppm}$ in the ${ }^{1} \mathrm{H}$ and ${ }^{13} \mathrm{C}$ NMR spectra, respectively), and coupling constants are reported in Hertz (Hz). Assignments given for the NMR spectra of the new compounds have been carried out by comparison with the NMR data of compounds $\mathbf{1 0}, \mathbf{2 5}, \mathbf{3 4}, \mathbf{3 7}, \mathbf{5 2}$, and $\mathbf{8 2}$, which in turn, were assigned on the basis of DEPT, COSY ${ }^{1} \mathrm{H} /{ }^{1} \mathrm{H}$ (standard procedures), and $\operatorname{COSY}{ }^{1} \mathrm{H} /{ }^{13} \mathrm{C}$ (gHSQC or gHMBC sequences) experiments. IR spectra were run on a Perkin-Elmer Spectrum RX I spectrophotometer. Absorption values are expressed as wavenumbers $\left(\mathrm{cm}^{-1}\right)$; only significant absorption bands are given. Column chromatography was performed on silica gel 60 AC.C (40-60 mesh, SDS, ref 2000027). Thin-layer chromatography was performed with aluminum-backed sheets with silica gel $60 \mathrm{~F}_{254}$ (Merck, ref 1.05554), and spots were visualized with UV light and $1 \%$ aqueous solution of $\mathrm{KMnO}_{4}$. High resolution mass spectra were carried out at the CCiTUB with a LC/MSD TOF Agilent Technologies spectrometer. The analytical samples of all of the compounds that were subjected to pharmacological evaluation were dried at $65{ }^{\circ} \mathrm{C} / 2$ Torr (standard conditions). 


\subsubsection{N-(tert-Butoxycarbonyl)-N-[(1-ethyl-1H-1,2,3-triazol-4-yl)methyl] amine 9}

To a solution of bromoethane $(0.53 \mathrm{~mL}, 774 \mathrm{mg}, 7.10 \mathrm{mmol})$ in $\mathrm{H}_{2} \mathrm{O} / \mathrm{DMF}$ 1:4 (50 $\mathrm{mL}$ ), $\mathrm{NaN}_{3}(502 \mathrm{mg}, 7.72 \mathrm{mmol}), \mathrm{Na}_{2} \mathrm{CO}_{3}(2.05 \mathrm{~g}, 19.3 \mathrm{mmol}$ ), ascorbic acid (907 mg, $5.15 \mathrm{mmol}), \mathrm{CuSO}_{4} \cdot 5 \mathrm{H}_{2} \mathrm{O}(641 \mathrm{mmg}, 2.57 \mathrm{mmol})$ and $N$-Boc-propargylamine, 7 (1.00 $\mathrm{g}, 6.44 \mathrm{mmol}$ ) were added. The reaction mixture was stirred at rt overnight, diluted with $20 \%$ aq. $\mathrm{NH}_{4} \mathrm{OH}(200 \mathrm{~mL})$, treated with solid EDTA (ca $5 \mathrm{~g}$ ) and extracted with EtOAc $(2 \times 150 \mathrm{~mL})$. The combined organic extracts were washed with $\mathrm{H}_{2} \mathrm{O}(3 \times 100 \mathrm{~mL})$, dried over anhydrous $\mathrm{Na}_{2} \mathrm{SO}_{4}$, and concentrated under reduced pressure. The resulting residue was washed with pentane $(3 \times 10 \mathrm{~mL})$ and dried under vacuum, to give compound 9 (1.14 g, 78\% yield) as a white solid; $R_{f} 0.57\left(\mathrm{CH}_{2} \mathrm{Cl}_{2} / \mathrm{MeOH} 9: 1\right)$; mp 77-78 ${ }^{\circ} \mathrm{C}$; IR (ATR) $v 3318(\mathrm{~N}-\mathrm{H}), 1685,1675(\mathrm{C}=\mathrm{O}) \mathrm{cm}^{-1} ;{ }^{1} \mathrm{H}$ NMR (400 MHz, $\left.\mathrm{CDCl}_{3}\right) \delta 1.40\left[\mathrm{~s}, 9 \mathrm{H}, \mathrm{C}\left(\mathrm{CH}_{3}\right)_{3}\right], 1.51\left(\mathrm{t}, J=7.2 \mathrm{~Hz}, 3 \mathrm{H}, 1-\mathrm{CH}_{2}-\mathrm{CH}_{3}\right), 4.35(\mathrm{q}, J=7.2$ $\left.\mathrm{Hz}, 2 \mathrm{H}, 1-\mathrm{CH}_{2}-\mathrm{CH}_{3}\right), 4.36$ (s, 2H, 4- $\mathrm{CH}_{2}-\mathrm{NH}$ ), 5.21 (broad s, 1H, NHBoc), 7.50 (s, $1 \mathrm{H}, 5-\mathrm{H}) ;{ }^{13} \mathrm{C} \mathrm{NMR}\left(100.6 \mathrm{MHz}, \mathrm{CDCl}_{3}\right) \delta 15.5\left(\mathrm{CH}_{3}, 1-\mathrm{CH}_{2}-\mathrm{CH}_{3}\right), 28.4\left[3 \mathrm{CH}_{3}\right.$, $\left.\mathrm{C}\left(\mathrm{CH}_{3}\right)_{3}\right], 36.2\left(\mathrm{CH}_{2}, 4-\mathrm{CH}_{2}-\mathrm{N}\right), 45.3\left(\mathrm{CH}_{2}, 1-\mathrm{CH}_{2}-\mathrm{CH}_{3}\right), 79.7\left[\mathrm{C}, \mathrm{C}\left(\mathrm{CH}_{3}\right)_{3}\right], 121.3$ (CH, C5), 145.6 (C, C4), 156.0 (C, NCOO); HRMS (ESI), calcd for $\left(\mathrm{C}_{10} \mathrm{H}_{18} \mathrm{~N}_{4} \mathrm{O}_{2}+\mathrm{H}^{+}\right)$ 227.1503 , found 227.1493 .

\subsection{2. $N$-(tert-Butoxycarbonyl)-N-[2-(1-ethyl-1H-1,2,3-triazol-4-yl)ethyl] amine 10}

It was prepared as described for 9. From bromoethane $(0.65 \mathrm{~mL}, 949 \mathrm{mg}, 8.71 \mathrm{mmol})$, $\mathrm{NaN}_{3}(618 \mathrm{mg}, 9.51 \mathrm{mmol}), \mathrm{Na}_{2} \mathrm{CO}_{3}(2.52 \mathrm{~g}, 23.8 \mathrm{mmol})$, ascorbic acid (1.12 g, 6.36 mmol), $\mathrm{CuSO}_{4} \cdot 5 \mathrm{H}_{2} \mathrm{O}$ (790 mg, $3.16 \mathrm{mmol}$ ), and $N$-Boc-3-butynylamine, 8 (1.34 g, 7.92 mmol), compound 10 (1.48 g, 78\% yield) was obtained as a white solid; $R_{f} 0.50$ $\left(\mathrm{CH}_{2} \mathrm{Cl}_{2} / \mathrm{MeOH} 9: 1\right) ; \mathrm{mp} 78-80{ }^{\circ} \mathrm{C}$; IR (ATR) v $3341(\mathrm{~N}-\mathrm{H}), 1692(\mathrm{C}=\mathrm{O}) \mathrm{cm}^{-1} ;{ }^{1} \mathrm{H}$ $\operatorname{NMR}\left(400 \mathrm{MHz}, \mathrm{CDCl}_{3}\right) \delta 1.40\left[\mathrm{~s}, 9 \mathrm{H}, \mathrm{C}\left(\mathrm{CH}_{3}\right)_{3}\right], 1.51(\mathrm{t}, J=7.6 \mathrm{~Hz}, 3 \mathrm{H}$, $1-\mathrm{CH}_{2}-\mathrm{CH}_{3}$ ), 2.87 (t, $J=6.4 \mathrm{~Hz}, 2 \mathrm{H}, 4-\mathrm{CH}_{2}-\mathrm{CH}_{2}-\mathrm{NH}$ ), 3.44 (dt, $J=J^{\prime}=6.4 \mathrm{~Hz}, 2 \mathrm{H}$, 4- $\mathrm{CH}_{2}-\mathrm{CH}_{2}-\mathrm{NH}$ ), 4.35 (q, $J=7.6 \mathrm{~Hz}, 2 \mathrm{H}, 1-\mathrm{CH}_{2}-\mathrm{CH}_{3}$ ), 5.04 (broad s, $1 \mathrm{H}, \mathrm{N} H \mathrm{Boc}$ ), $7.35(\mathrm{~s}, 1 \mathrm{H}, 5-\mathrm{H}) ;{ }^{13} \mathrm{C} \mathrm{NMR}\left(100.6 \mathrm{MHz}, \mathrm{CDCl}_{3}\right) \delta 15.6\left(\mathrm{CH}_{3}, 1-\mathrm{CH}_{2}-\mathrm{CH}_{3}\right), 26.3$ $\left(\mathrm{CH}_{2}, 4-\mathrm{CH}_{2}-\mathrm{CH}_{2}-\mathrm{N}\right), 28.5\left[3 \mathrm{CH}_{3}, \mathrm{C}\left(\mathrm{CH}_{3}\right)_{3}\right], 39.9\left(\mathrm{CH}_{2}, 4-\mathrm{CH}_{2}-\mathrm{CH}_{2}-\mathrm{N}\right), 45.2\left(\mathrm{CH}_{2}\right.$, 1- $\left.\mathrm{CH}_{2}-\mathrm{CH}_{3}\right), 79.2\left[\mathrm{C}, \mathrm{C}\left(\mathrm{CH}_{3}\right)_{3}\right], 120.8(\mathrm{CH}, \mathrm{C} 5), 145.5(\mathrm{C}, \mathrm{C} 4), 156.1$ (C, NCOO); HRMS (ESI), calcd for $\left(\mathrm{C}_{11} \mathrm{H}_{20} \mathrm{~N}_{4} \mathrm{O}_{2}+\mathrm{H}^{+}\right)$241.1659, found 241.1654 .

\subsection{3. $N$-(tert-Butoxycarbonyl)-N-[2-(1-benzyl-1H-1,2,3-triazol-4-yl)ethyl] amine 11}


It was prepared as described for 9. From benzyl bromide $(0.75 \mathrm{~mL}, 1.08 \mathrm{~g}, 6.31 \mathrm{mmol})$, $\mathrm{NaN}_{3}(462 \mathrm{mg}, 7.11 \mathrm{mmol}), \mathrm{Na}_{2} \mathrm{CO}_{3}(1.88 \mathrm{~g}, 17.7 \mathrm{mmol}$ ), ascorbic acid (834 mg, 4.74 mmol), $\mathrm{CuSO}_{4} \cdot 5 \mathrm{H}_{2} \mathrm{O}(590 \mathrm{mg}, 2.36 \mathrm{mmol}$ ), and $\mathrm{N}$-Boc-3-butynylamine, 8 (1.00 g, 5.91 mmol), compound 11 (1.27 g, 71\% yield) was obtained as a white solid; $R_{f} 0.58$ $\left(\mathrm{CH}_{2} \mathrm{Cl}_{2} / \mathrm{MeOH} / 50 \%\right.$ aq. $\mathrm{NH}_{4} \mathrm{OH}$ 9.5:0.5:0.05); mp 103-105 ${ }^{\circ} \mathrm{C}$; IR (ATR) v 3407 $(\mathrm{N}-\mathrm{H}), 1688(\mathrm{C}=\mathrm{O}) \mathrm{cm}^{-1} ;{ }^{1} \mathrm{H} \mathrm{NMR}\left(400 \mathrm{MHz}, \mathrm{CDCl}_{3}\right) \delta 1.38\left[\mathrm{~s}, 9 \mathrm{H}, \mathrm{C}\left(\mathrm{CH}_{3}\right)_{3}\right], 2.84(\mathrm{t}$, $\left.J=6.8 \mathrm{~Hz}, 2 \mathrm{H}, 4-\mathrm{CH}_{2}-\mathrm{CH}_{2}-\mathrm{NH}\right), 3.41\left(\mathrm{td}, J=6.8 \mathrm{~Hz}, J^{\prime}=6.0 \mathrm{~Hz}, 2 \mathrm{H}\right.$, 4- $\mathrm{CH}_{2}-\mathrm{CH}_{2}-\mathrm{NH}$ ), 5.04 (broad s, $1 \mathrm{H}, \mathrm{N} H \mathrm{Boc}$ ), 5.46 (s, 2H, 1-CH $-\mathrm{Ph}$ ), 7.22 [ddm, $J=$ 7.6 Hz, $J^{\prime}=2.0 \mathrm{~Hz}, 2 \mathrm{H}, \mathrm{Ph}-\mathrm{C} 2(6)-H$ ], 7.29-7.37 [complex signal, 4H, 5-H, $\mathrm{Ph}-\mathrm{C} 3(5)-H, \mathrm{Ph}-\mathrm{C} 4-H] ;{ }^{13} \mathrm{C} \mathrm{NMR}\left(100.6 \mathrm{MHz}, \mathrm{CDCl}_{3}\right) \delta 26.3\left(\mathrm{CH}_{2}, 4-\mathrm{CH}_{2}-\mathrm{CH}_{2} \mathrm{~N}\right)$, $28.4\left[3 \mathrm{CH}_{3}, \mathrm{C}\left(\mathrm{CH}_{3}\right)_{3}\right], 39.8\left(\mathrm{CH}_{2}, 4-\mathrm{CH}_{2}-\mathrm{CH}_{2}-\mathrm{N}\right), 54.1\left(\mathrm{CH}_{2}, 1-\mathrm{CH}_{2}-\mathrm{Ph}\right), 79.2[\mathrm{C}$, $\left.C\left(\mathrm{CH}_{3}\right)_{3}\right], 121.4(\mathrm{CH}, \mathrm{C} 5), 128.1(2 \mathrm{CH}), 128.8(\mathrm{CH}), 129.2(2 \mathrm{CH})(\mathrm{Ph}-\mathrm{CH}), 134.8(\mathrm{C}$, $\mathrm{Ph}-\mathrm{C} 1), 145.9$ (C, C4), 156.0 (C, NCOO); HRMS (ESI), calcd for $\left(\mathrm{C}_{16} \mathrm{H}_{22} \mathrm{~N}_{4} \mathrm{O}_{2}+\mathrm{H}^{+}\right)$ 303.1816, found 303.1813 .

\subsection{4. $\mathrm{N}$-(tert-Butoxycarbonyl)-N-[2-(1-phenethyl-1H-1,2,3-triazol-4-yl)ethyl]amine 12}

It was prepared as described for 9. From phenethyl bromide $(0.89 \mathrm{~mL}, 1.21 \mathrm{~g}, 6.52$ mmol), $\mathrm{NaN}_{3}$ (462 mg, $7.11 \mathrm{mmol}$ ), $\mathrm{Na}_{2} \mathrm{CO}_{3}$ (1.88 g, $17.7 \mathrm{mmol}$ ), ascorbic acid (834 $\mathrm{mg}, 4.74 \mathrm{mmol}), \mathrm{CuSO}_{4} \cdot 5 \mathrm{H}_{2} \mathrm{O}(590 \mathrm{mg}, 2.36 \mathrm{mmol})$, and $\mathrm{N}$-Boc-3-butynylamine, 8 (1.00 g, $5.91 \mathrm{mmol})$, compound 12 (908 $\mathrm{mg}, 49 \%$ yield) was obtained as a white solid; $R_{f} 0.52\left(\mathrm{CH}_{2} \mathrm{Cl}_{2} / \mathrm{MeOH} / 50 \%\right.$ aq. $\mathrm{NH}_{4} \mathrm{OH}$ 9.5:0.5:0.05); mp 123-125 ${ }^{\circ} \mathrm{C}$; IR (ATR) $v$ $3392(\mathrm{~N}-\mathrm{H}), 1688(\mathrm{C}=\mathrm{O}) \mathrm{cm}^{-1} ;{ }^{1} \mathrm{H} \mathrm{NMR}\left(400 \mathrm{MHz}, \mathrm{CDCl}_{3}\right) \delta 1.43\left[\mathrm{~s}, 9 \mathrm{H}, \mathrm{C}\left(\mathrm{CH}_{3}\right)_{3}\right]$, 2.85 (t, $\left.J=6.8 \mathrm{~Hz}, 2 \mathrm{H}, 4-\mathrm{CH}_{2}-\mathrm{CH}_{2}-\mathrm{N}\right), 3.19$ (t, $J=7.2 \mathrm{~Hz} 2 \mathrm{H}, 1-\mathrm{CH}_{2}-\mathrm{CH}_{2}-\mathrm{Ph}$ ), 3.42 (m, 2H, 4- $\left.\mathrm{CH}_{2}-\mathrm{CH}_{2}-\mathrm{N}\right), 4.55$ (t, $\left.J=7.2 \mathrm{~Hz}, 2 \mathrm{H}, 1-\mathrm{CH}_{2}-\mathrm{CH}_{2}-\mathrm{Ph}\right), 4.95$ (br s, $1 \mathrm{H}$, NHBoc), 7.09 [dm, $J=6.8 \mathrm{~Hz}, 2 \mathrm{H}, \mathrm{Ph}-\mathrm{C} 2(6)-H$ ], 7.22-7.32 [complex signal, 4H, 5$\mathrm{H}, \quad \mathrm{Ph}-\mathrm{C} 3(5)-H, \quad \mathrm{Ph}-\mathrm{C} 4-H] ;{ }^{13} \mathrm{C}$ NMR (100.6 MHz, $\left.\mathrm{CDCl}_{3}\right) \quad \delta \quad 26.2 \quad\left(\mathrm{CH}_{2}\right.$, $\left.4-\mathrm{CH}_{2}-\mathrm{CH}_{2}-\mathrm{N}\right), 28.5\left[3 \mathrm{CH}_{3}, \mathrm{C}\left(\mathrm{CH}_{3}\right)_{3}\right], 36.9\left(\mathrm{CH}_{2}, 1-\mathrm{CH}_{2}-\mathrm{CH}_{2}-\mathrm{Ph}\right), 40.0\left(\mathrm{CH}_{2}\right.$, 4- $\left.\mathrm{CH}_{2}-\mathrm{CH}_{2}-\mathrm{N}\right), 51.6\left(\mathrm{CH}_{2}, 1-\mathrm{CH}_{2}-\mathrm{CH}_{2}-\mathrm{Ph}\right), 79.3\left[\mathrm{C}, \mathrm{C}\left(\mathrm{CH}_{3}\right)_{3}\right], 121.9(\mathrm{CH}, \mathrm{C} 5)$, $127.2(\mathrm{CH}), 128.8(2 \mathrm{CH}), 128.9(2 \mathrm{CH})(\mathrm{Ph}-\mathrm{CH}), 137.2(\mathrm{C}, \mathrm{Ph}-\mathrm{C} 1), 145.2(\mathrm{C}, \mathrm{C} 4)$, 156.1 (C, NCOO); HRMS (ESI), calcd for $\left(\mathrm{C}_{17} \mathrm{H}_{24} \mathrm{~N}_{4} \mathrm{O}_{2}+\mathrm{H}^{+}\right)$317.1972, found 317.1968 . 


\subsection{5. $N$-(tert-Butoxycarbonyl)-N-[(1-methyl-1H-1,2,3-triazol-4-yl)methyl] amine 13}

It was prepared as described for 9. From iodomethane $(0.44 \mathrm{~mL}, 1.00 \mathrm{~g}, 7.07 \mathrm{mmol})$, $\mathrm{NaN}_{3}(502 \mathrm{mg}, 7.72 \mathrm{mmol}), \mathrm{Na}_{2} \mathrm{CO}_{3}(2.05 \mathrm{~g}, 19.3 \mathrm{mmol}$ ), ascorbic acid (907 mg, 5.15 mmol), $\mathrm{CuSO}_{4} \cdot 5 \mathrm{H}_{2} \mathrm{O}(641 \mathrm{mg}, 2.57 \mathrm{mmol})$, and $N$-Boc-propargylamine, 7 (1.00 g, 6.44 mmol), compound 13 (938 mg, 69\% yield) was obtained as a white solid; $R_{f} 0.87$ $\left(\mathrm{CH}_{2} \mathrm{Cl}_{2} / \mathrm{MeOH} / 50 \%\right.$ aq. $\mathrm{NH}_{4} \mathrm{OH}$ 9.5:0.5:0.05); mp 102-104 ${ }^{\circ} \mathrm{C}$; IR (ATR) $v 3379$ $(\mathrm{N}-\mathrm{H}), 1679(\mathrm{C}=\mathrm{O}) \mathrm{cm}^{-1} ;{ }^{1} \mathrm{H}$ NMR $\left(400 \mathrm{MHz}, \mathrm{CDCl}_{3}\right) \delta 1.41\left[\mathrm{~s}, 9 \mathrm{H}, \mathrm{C}\left(\mathrm{CH}_{3}\right)_{3}\right], 4.05(\mathrm{~s}$, $\left.3 \mathrm{H}, 1-\mathrm{CH}_{3}\right), 4.35\left(\mathrm{~d}, J=6.0 \mathrm{~Hz}, 2 \mathrm{H}, 4-\mathrm{CH}_{2}-\mathrm{N}\right.$ ), 5.17 (br s, $\left.1 \mathrm{H}, \mathrm{N} H \mathrm{Boc}\right), 7.48$ (s, $1 \mathrm{H}$, $5-H) ;{ }^{13} \mathrm{C}$ NMR $\left(100.6 \mathrm{MHz}, \mathrm{CDCl}_{3}\right) \delta: 28.5\left[3 \mathrm{CH}_{3}, \mathrm{C}\left(\mathrm{CH}_{3}\right)_{3}\right], 36.2\left(\mathrm{CH}_{2}, 4-\mathrm{CH}_{2}-\mathrm{N}\right)$, $36.7\left(\mathrm{CH}_{3}, 1-\mathrm{CH}_{3}\right), 79.7\left[\mathrm{C}, \mathrm{C}\left(\mathrm{CH}_{3}\right)_{3}\right], 122.9(\mathrm{CH}, \mathrm{C} 5), 145.9(\mathrm{C}, \mathrm{C} 4), 156.0(\mathrm{C}$, NCOO); HRMS (ESI), calcd for $\left(\mathrm{C}_{9} \mathrm{H}_{16} \mathrm{~N}_{4} \mathrm{O}_{2}+\mathrm{H}^{+}\right)$213.1346, found 213.1336.

\subsection{6. $N$-(tert-Butoxycarbonyl)-N-[2-(1-phenyl-1H-1,2,3-triazol-4-yl)ethyl]amine 14}

It was prepared as described for 9. From phenyl azide $(0.5 \mathrm{M}$ solution in tert-butyl methyl ether, $26.2 \mathrm{~mL}, 13.1 \mathrm{mmol}), \mathrm{Na}_{2} \mathrm{CO}_{3}(3.47 \mathrm{~g}, 32.7 \mathrm{mmol})$, ascorbic acid (1.54 g, $8.74 \mathrm{mmol}), \mathrm{CuSO}_{4} \cdot 5 \mathrm{H}_{2} \mathrm{O}(1.09 \mathrm{mmg}, 4.36 \mathrm{mmol})$, and $N$-Boc-3-butynylamine, 8 (2.00 g, $11.8 \mathrm{mmol}$ ), compound 14 ( $1.89 \mathrm{~g}$, 56\% yield) was obtained as a yellow solid; $R_{f} 0.90$ $\left(\mathrm{CH}_{2} \mathrm{Cl}_{2} / \mathrm{MeOH} / 50 \%\right.$ aq. $\mathrm{NH}_{4} \mathrm{OH}$ 9:1:0.05); mp 127-129 ${ }^{\circ} \mathrm{C}$; IR (ATR) $v 3297$ $(\mathrm{N}-\mathrm{H}), 1712(\mathrm{C}=\mathrm{O}) \mathrm{cm}^{-1} ;{ }^{1} \mathrm{H} \mathrm{NMR}\left(400 \mathrm{MHz}, \mathrm{CDCl}_{3}\right) \delta 1.43\left[\mathrm{~s}, 9 \mathrm{H}, \mathrm{C}\left(\mathrm{CH}_{3}\right)_{3}\right], 3.00(\mathrm{t}$, $\left.J=6.8 \mathrm{~Hz}, 2 \mathrm{H}, 4-\mathrm{CH}_{2}-\mathrm{CH}_{2}-\mathrm{N}\right), 3.55\left(\mathrm{td}, J=6.8 \mathrm{~Hz}, J^{\prime}=6.0 \mathrm{~Hz}, 2 \mathrm{H}\right.$, 4- $\mathrm{CH}_{2}-\mathrm{CH}_{2}-\mathrm{N}$ ), 5.02 (br s, 1H, NHBoc), 7.43 (tt, $J=7.4 \mathrm{~Hz}, J^{\prime}=1.2 \mathrm{~Hz}, 1 \mathrm{H}$, $\mathrm{Ph}-\mathrm{C} 4-H), 7.52[\mathrm{~m}, 2 \mathrm{H}, \mathrm{Ph}-\mathrm{C} 3(5)-H], 7.71\left[\mathrm{ddd}, J=8.8 \mathrm{~Hz}, J^{\prime}=2.4 \mathrm{~Hz}, J^{\prime \prime}=1.2 \mathrm{~Hz}\right.$, $2 \mathrm{H}, \mathrm{Ph}-\mathrm{C} 2(6)-H], 7.81(\mathrm{~s}, 1 \mathrm{H}, 5-\mathrm{H}) ;{ }^{13} \mathrm{C} \mathrm{NMR}\left(100.6 \mathrm{MHz}, \mathrm{CDCl}_{3}\right) \delta 26.4\left(\mathrm{CH}_{2}\right.$, $\left.4-\mathrm{CH}_{2}-\mathrm{CH}_{2}-\mathrm{N}\right), 28.5 \quad\left[3 \mathrm{CH}_{3}, \mathrm{C}\left(\mathrm{CH}_{3}\right)_{3}\right], 39.9\left(\mathrm{CH}_{2}, 4-\mathrm{CH}_{2}-\mathrm{CH}_{2}-\mathrm{N}\right), 79.4 \quad[\mathrm{C}$, $\left.C\left(\mathrm{CH}_{3}\right)_{3}\right], 119.8(\mathrm{CH}, \mathrm{Ph}-\mathrm{C} 4), 120.5(\mathrm{CH}, \mathrm{C} 5), 128.7(2 \mathrm{CH}), 129.9(2 \mathrm{CH})[\mathrm{Ph}-\mathrm{C} 2(6)$, Ph-C3(5)], 137.2 (C, Ph-C1), 146.3 (C, C4), 156.1 (C, NCOO); HRMS (ESI), calcd for $\left(\mathrm{C}_{15} \mathrm{H}_{20} \mathrm{~N}_{4} \mathrm{O}_{2}+\mathrm{Na}^{+}\right)$311.1478, found 311.1476.

\subsection{7. $N$-[(1-Benzyl-1H-1,2,3-triazol-4-yl)methyl]-N-(tert-butoxycarbonyl)amine $\mathbf{1 5}$}

It was prepared as described for 9. From benzyl bromide (1.04 mL, $1.50 \mathrm{~g}, 8.74 \mathrm{mmol})$, $\mathrm{NaN}_{3}(622 \mathrm{mg}, 9.57 \mathrm{mmol}), \mathrm{Na}_{2} \mathrm{CO}_{3}(2.53 \mathrm{~g}, 23.9 \mathrm{mmol})$, ascorbic acid (1.12 g, 6.36 mmol), $\mathrm{CuSO}_{4} \cdot 5 \mathrm{H}_{2} \mathrm{O}$ (796 mg, $3.19 \mathrm{mmol}$ ), and $N$-Boc-propargylamine, 7 (1.24 g, 7.99 mmol), compound 15 (1.68 g, 68\% yield) was obtained as a white solid; $R_{f} 0.62$ 
$\left(\mathrm{CH}_{2} \mathrm{Cl}_{2} / \mathrm{MeOH} / \mathrm{NH}_{4} \mathrm{OH}\right.$ 9.75:0.25:0.05); mp 97-99 ${ }^{\circ} \mathrm{C}$; IR (ATR) v 3400, 3302, $3105,3059(\mathrm{~N}-\mathrm{H}), 1684,1679(\mathrm{C}=\mathrm{O}) \mathrm{cm}^{-1}{ }^{1} \mathrm{H}$ NMR $\left(400 \mathrm{MHz}, \mathrm{CDCl}_{3}\right) \delta 1.32[\mathrm{~s}, 9 \mathrm{H}$, $\left.\mathrm{C}\left(\mathrm{CH}_{3}\right)_{3}\right], 4.27\left(\mathrm{~d}, J=6.0 \mathrm{~Hz}, 2 \mathrm{H}, 4-\mathrm{CH}_{2}-\mathrm{N}\right), 5.06($ br s, $1 \mathrm{H}, \mathrm{NHBoc}), 5.40(\mathrm{~s}, 2 \mathrm{H}$, 1- $\mathrm{CH}_{2}-\mathrm{Ph}$ ), $7.26[\mathrm{~m}, 2 \mathrm{H}, \mathrm{Ph}-\mathrm{C} 2(6)-H]$, 7.30-7.40 [complex signal, $3 \mathrm{H}, \mathrm{Ph}-\mathrm{C} 3(5)-H$, $\mathrm{Ph}-\mathrm{C} 4-H], 7.43(\mathrm{~s}, 1 \mathrm{H}, 5-\mathrm{H}) ;{ }^{13} \mathrm{C} \mathrm{NMR}\left(100.6 \mathrm{MHz}, \mathrm{CDCl}_{3}\right) \delta 28.4\left[3 \mathrm{CH}_{3}, \mathrm{C}\left(\mathrm{CH}_{3}\right)_{3}\right]$, $36.2\left(\mathrm{CH}_{2}, 4-\mathrm{CH}_{2}-\mathrm{N}\right), 54.2\left(\mathrm{CH}_{2}, 1-\mathrm{CH}_{2}-\mathrm{Ph}\right), 79.8\left[\mathrm{C}, \mathrm{C}\left(\mathrm{CH}_{3}\right)_{3}\right], 121.9(\mathrm{CH}, \mathrm{C} 5)$, $128.2(2 \mathrm{CH}), 128.8(\mathrm{CH}), 129.2(2 \mathrm{CH})(\mathrm{Ph}-\mathrm{CH}), 134.7(\mathrm{C}, \mathrm{Ph}-\mathrm{C} 1), 146.0(\mathrm{C}, \mathrm{C} 4)$, 155.9 (C, NCOO); HRMS (ESI), calcd for $\left(\mathrm{C}_{15} \mathrm{H}_{20} \mathrm{~N}_{4} \mathrm{O}_{2}+\mathrm{Na}^{+}\right)$311.1478, found 311.1487 .

\subsection{8. $N$-(tert-Butoxycarbonyl)-N-[(1-phenethyl-1H-1,2,3-triazol-4-yl)methyl] amine 16}

It was prepared as described for 9. From phenethyl bromide $(1.11 \mathrm{~mL}, 1.50 \mathrm{~g}, 8.13$ mmol), $\mathrm{NaN}_{3}(574 \mathrm{mg}, 8.83 \mathrm{mmol}), \mathrm{Na}_{2} \mathrm{CO}_{3}(2.34 \mathrm{~g}, 22.1 \mathrm{mmol})$, ascorbic acid (1.04 g, $5.91 \mathrm{mmol}), \mathrm{CuSO}_{4} \cdot 5 \mathrm{H}_{2} \mathrm{O}(735 \mathrm{mg}, 2.94 \mathrm{mmol})$, and $N$-Boc-propargylamine, 7 (1.00 g, $6.44 \mathrm{mmol})$, compound 16 (1.43 g, 73\% yield) was obtained as a white solid; $R_{f} 0.57$ $\left(\mathrm{CH}_{2} \mathrm{Cl}_{2} / \mathrm{MeOH} / \mathrm{NH}_{4} \mathrm{OH}\right.$ 9.75:0.25:0.05); $\mathrm{mp} \mathrm{98-100}{ }^{\circ} \mathrm{C}$; IR (ATR) v 3410, 3111, 3069, $3054(\mathrm{~N}-\mathrm{H}), 1684(\mathrm{C}=\mathrm{O}) \mathrm{cm}^{-1} ;{ }^{1} \mathrm{H}$ NMR (400 MHz, $\left.\mathrm{CDCl}_{3}\right) \delta 1.43[\mathrm{~s}, 9 \mathrm{H}$, $\left.\mathrm{C}\left(\mathrm{CH}_{3}\right)_{3}\right], 3.19\left(\mathrm{t}, J=7.2 \mathrm{~Hz}, 2 \mathrm{H}, 1-\mathrm{CH}_{2}-\mathrm{CH}_{2}-\mathrm{Ph}\right), 4.34(\mathrm{~d}, J=6.0 \mathrm{~Hz}, 2 \mathrm{H}$, 4- $\left.\mathrm{CH}_{2}-\mathrm{N}\right), 4.56$ (t, $J=7.2 \mathrm{~Hz}, 2 \mathrm{H}, 1-\mathrm{CH}_{2}-\mathrm{CH}_{2}-\mathrm{Ph}$ ), 5.14 (br s, 1H, NHBoc), 7.10 $[\mathrm{dm}, J=8.4 \mathrm{~Hz}, 2 \mathrm{H}, \mathrm{Ph}-\mathrm{C} 2(6)-H]$ ] 7.21-7.32 [complex signal, 4H, 5-H, $\mathrm{Ph}-\mathrm{C} 3(5)-H$, $\mathrm{Ph}-\mathrm{C} 4-\mathrm{H}] ;{ }^{13} \mathrm{C}$ NMR $\left(100.6 \mathrm{MHz}, \mathrm{CDCl}_{3}\right) \delta 28.5\left[3 \mathrm{CH}_{3}, \mathrm{C}\left(\mathrm{CH}_{3}\right)_{3}\right], 36.2\left(\mathrm{CH}_{2}\right.$, 4- $\left.\mathrm{CH}_{2}-\mathrm{N}\right), 36.8\left(\mathrm{CH}_{2}, 1-\mathrm{CH}_{2}-\mathrm{CH}_{2}-\mathrm{Ph}\right), 51.7\left(\mathrm{CH}_{2}, 1-\mathrm{CH}_{2}-\mathrm{CH}_{2}-\mathrm{Ph}\right), 79.7$ [C, $\left.C\left(\mathrm{CH}_{3}\right)_{3}\right], 122.2(\mathrm{CH}, \mathrm{C} 5), 127.2(\mathrm{CH}), 128.7(2 \mathrm{CH}), 128.9(2 \mathrm{CH})(\mathrm{Ph}-\mathrm{CH}), 137.0(\mathrm{C}$, $\mathrm{Ph}-\mathrm{C} 1), 145.3$ (C, C4), 155.9 (C, NCOO); HRMS (ESI), calcd for $\left(\mathrm{C}_{16} \mathrm{H}_{22} \mathrm{~N}_{4} \mathrm{O}_{2}+\mathrm{H}^{+}\right)$ 303.1816, found 303.1827.

\subsection{9. $N$-(tert-Butoxycarbonyl)-N-[(1-ethyl-1H-1,2,3-triazol-4-yl)methyl]-N-} methylamine 17

A solution of $9(634 \mathrm{mg}, 2.80 \mathrm{mmol})$ in anhydrous THF $(6.5 \mathrm{~mL})$ was cooled to $0{ }^{\circ} \mathrm{C}$ and treated with $\mathrm{NaH}(60 \%$ suspension in mineral oil, $246 \mathrm{mg}, 6.15 \mathrm{mmol})$ and iodomethane $(0.19 \mathrm{~mL}, 433 \mathrm{mg}, 3.05 \mathrm{mmol})$. The reaction mixture was stirred at $\mathrm{rt}$ for 3 h, cooled to $0{ }^{\circ} \mathrm{C}$, diluted dropwise with sat. aq. $\mathrm{NH}_{4} \mathrm{Cl}(40 \mathrm{~mL})$, and extracted with EtOAc $(3 \times 40 \mathrm{~mL})$. The combined organic extracts were dried over anhydrous $\mathrm{Na}_{2} \mathrm{SO}_{4}$ 
and evaporated under reduced pressure. The resulting residue was washed with pentane $(3 \times 10 \mathrm{~mL})$ and dried under vacuum, to give $17(654 \mathrm{mg}, 97 \%$ yield $)$ as a colorless oil; $R_{f} 0.64\left(\mathrm{CH}_{2} \mathrm{Cl}_{2} / \mathrm{MeOH} 9: 1\right)$; IR (ATR) $v$ 1688, $1677(\mathrm{C}=\mathrm{O}) \mathrm{cm}^{-1} ;{ }^{1} \mathrm{H}$ NMR (400 $\left.\mathrm{MHz}, \mathrm{CDCl}_{3}\right) \delta 1.40\left[\mathrm{~s}, \mathrm{C}\left(\mathrm{CH}_{3}\right)_{3}\right.$ minor rotamer], 1.42 [s, $\mathrm{C}\left(\mathrm{CH}_{3}\right)_{3}$ major rotamer], 1.50 (t, $J=7.2 \mathrm{~Hz}, 1-\mathrm{CH}_{2}-\mathrm{CH}_{3}$ minor rotamer), 1.51 (t, $J=7.2 \mathrm{~Hz}, 1-\mathrm{CH}_{2}-\mathrm{CH}_{3}$ major rotamer), $2.87\left(\mathrm{~s}, 3 \mathrm{H}, \mathrm{N}-\mathrm{CH}_{3}\right), 4.39\left(\mathrm{q}, J=7.2 \mathrm{~Hz}, 1-\mathrm{CH}_{2}-\mathrm{CH}_{3}\right.$ minor rotamer), 4.40 (q, $J=7.2 \mathrm{~Hz}, 1-\mathrm{CH}_{2}-\mathrm{CH}_{3}$ major rotamer), $4.49\left(\mathrm{~s}, 4-\mathrm{CH}_{2}-\mathrm{N}\right.$ ), 7.37 (br s, 5-H minor rotamer), 7.49 (s, 5- $\mathrm{H}$ major rotamer); HRMS (ESI), calcd for $\left(\mathrm{C}_{11} \mathrm{H}_{20} \mathrm{~N}_{4} \mathrm{O}_{2}+\mathrm{H}^{+}\right)$ 241.1659 , found 241.1654 .

\subsubsection{0. $N$-(tert-Butoxycarbonyl)-N-[2-(1-ethyl-1H-1,2,3-triazol-4-yl)ethyl]-N- methylamine 18}

It was prepared as described for 17. From compound 10 (1.70 g, $7.08 \mathrm{mmol}), \mathrm{NaH}$ (60\% supension in mineral oil, $424 \mathrm{mg}, 10.6 \mathrm{mmol})$, and iodomethane $(0.48 \mathrm{~mL}, 1.09 \mathrm{~g}$, $7.71 \mathrm{mmol})$, stirring at $\mathrm{rt}$ for $2 \mathrm{~h}$, compound $\mathbf{1 8}(1.71 \mathrm{mg}, 95 \%$ yield $)$ was obtained as a colorless oil; $R_{f} 0.58\left(\mathrm{CH}_{2} \mathrm{Cl}_{2} / \mathrm{MeOH} 9: 1\right)$; IR (ATR) $v 1690(\mathrm{C}=\mathrm{O}) \mathrm{cm}^{-1} ;{ }^{1} \mathrm{H} \mathrm{NMR}$ $\left(400 \mathrm{MHz}, \mathrm{CDCl}_{3}\right) \delta 1.41\left[\mathrm{~s}, 9 \mathrm{H}, \mathrm{C}\left(\mathrm{CH}_{3}\right)_{3}\right], 1.51\left(\mathrm{t}, J=7.2 \mathrm{~Hz}, 3 \mathrm{H}, 1-\mathrm{CH}_{2}-\mathrm{CH}_{3}\right), 2.82$ $\left(\mathrm{s}, 3 \mathrm{H}, \mathrm{N}-\mathrm{CH}_{3}\right), 2.93\left(\mathrm{~m}, 2 \mathrm{H}, 4-\mathrm{CH}_{2}-\mathrm{CH}_{2}-\mathrm{N}\right), 3.52($ br t, $J=6.8 \mathrm{~Hz}, 2 \mathrm{H}$, 4- $\mathrm{CH}_{2}-\mathrm{CH}_{2}-\mathrm{N}$ ), 4.35 (q, $\left.J=7.2 \mathrm{~Hz}, 2 \mathrm{H}, 1-\mathrm{CH}_{2}-\mathrm{CH}_{3}\right), 7.28$ (br s), 7.40 (br s) (5-H);

${ }^{13} \mathrm{C}$ NMR $\left(100.6 \mathrm{MHz}, \mathrm{CDCl}_{3}\right) \delta 15.6\left(\mathrm{CH}_{3}, 1-\mathrm{CH}_{2}-\mathrm{CH}_{3}\right), 24.3\left(\mathrm{CH}_{2}\right), 24.7\left(\mathrm{CH}_{2}\right)$ $\left(4-\mathrm{CH}_{2}-\mathrm{CH}_{2}-\mathrm{N}\right), 28.5\left[3 \mathrm{CH}_{3}, \mathrm{C}\left(\mathrm{CH}_{3}\right)_{3}\right], 34.4\left(\mathrm{CH}_{3}, \mathrm{~N}-\mathrm{CH}_{3}\right), 45.2\left(\mathrm{CH}_{2}, 1-\mathrm{CH}_{2}-\mathrm{CH}_{3}\right)$, $48.0\left(\mathrm{CH}_{2}\right), 48.9\left(\mathrm{CH}_{2}\right)\left(4-\mathrm{CH}_{2}-\mathrm{CH}_{2}-\mathrm{N}\right), 79.5\left[\mathrm{C}, \mathrm{C}\left(\mathrm{CH}_{3}\right)_{3}\right], 120.8(\mathrm{CH}, \mathrm{C} 5), 145.2(\mathrm{br}$ C, C4), 155.8 (br C, NCOO); HRMS (ESI), calcd for $\left(\mathrm{C}_{12} \mathrm{H}_{22} \mathrm{~N}_{4} \mathrm{O}_{2}+\mathrm{H}^{+}\right)$255.1816, found 255.1815 .

\subsubsection{N-(tert-Butoxycarbonyl)-N-[2-(1-benzyl-1H-1,2,3-triazol-4-yl)ethyl]-N- methylamine 19}

It was prepared as described for 17. From compound 11 (3.67 g, $12.1 \mathrm{mmol}), \mathrm{NaH}$ (60\% supension in mineral oil, $0.73 \mathrm{~g}, 18.2 \mathrm{mmol})$, and iodomethane $(0.83 \mathrm{~mL}, 1.89 \mathrm{~g}$, $13.3 \mathrm{mmol}$ ), stirring at $\mathrm{rt}$ for $3 \mathrm{~h}$, compound 19 (3.64 g, 95\% yield) was obtained as a yellowish oil; $R_{f} 0.60\left(\mathrm{CH}_{2} \mathrm{Cl}_{2} / \mathrm{MeOH} / 50 \%\right.$ aq. $\mathrm{NH}_{4} \mathrm{OH}$ 9.5:0.5:0.05); IR (ATR) $v$ $1687(\mathrm{C}=\mathrm{O}) \mathrm{cm}^{-1} ;{ }^{1} \mathrm{H} \mathrm{NMR}\left(400 \mathrm{MHz}, \mathrm{CDCl}_{3}\right) \delta 1.37\left[\mathrm{~s}, 9 \mathrm{H}, \mathrm{C}\left(\mathrm{CH}_{3}\right)_{3}\right], 2.78(\mathrm{~s}, 3 \mathrm{H}$, $\left.\mathrm{N}-\mathrm{CH}_{3}\right), 2.90\left(\mathrm{~m}, 2 \mathrm{H}, 4-\mathrm{CH}_{2}-\mathrm{CH}_{2}-\mathrm{N}\right), 3.48\left(\mathrm{t}, \mathrm{J}=6.8 \mathrm{~Hz}, 2 \mathrm{H}, 4-\mathrm{CH}_{2}-\mathrm{CH}_{2}-\mathrm{N}\right), 5.45$ 
(s, 2H, 1- $\mathrm{CH}_{2}-\mathrm{Ph}$ ), 7.20-7.37 (complex signal, 6H, 5-H, Ph-CH); ${ }^{13} \mathrm{C}$ NMR $(100.6$ $\left.\mathrm{MHz}, \mathrm{CDCl}_{3}\right) \delta 24.2\left(\mathrm{CH}_{2}\right), 24.7\left(\mathrm{CH}_{2}\right)\left(4-\mathrm{CH}_{2}-\mathrm{CH}_{2}-\mathrm{N}\right), 28.4\left[3 \mathrm{CH}_{3}, \mathrm{C}\left(\mathrm{CH}_{3}\right)_{3}\right], 34.4$ $\left[\mathrm{CH}_{3}, \mathrm{~N}-\mathrm{CH}_{3}\right), 48.0\left(\mathrm{CH}_{2}\right), 48.7\left(\mathrm{CH}_{2}\right)\left(4-\mathrm{CH}_{2}-\mathrm{CH}_{2}-\mathrm{N}\right), 54.1\left(\mathrm{CH}_{2}, 1-\mathrm{CH}_{2}-\mathrm{Ph}\right), 79.5$ $\left[\mathrm{C}, \mathrm{C}\left(\mathrm{CH}_{3}\right)_{3}\right], 121.4$ (br $\left.\mathrm{CH}, \mathrm{C} 5\right), 128.1(2 \mathrm{CH}), 128.7(\mathrm{CH}), 129.1(2 \mathrm{CH})(\mathrm{Ph}-\mathrm{CH})$, 134.9 (C, Ph-C1), 145.6 (br C, C4), 155.7 (C, NCOO); HRMS (ESI), calcd for $\left(\mathrm{C}_{17} \mathrm{H}_{24} \mathrm{~N}_{4} \mathrm{O}_{2}+\mathrm{H}^{+}\right)$317.1972, found 317.1964.

\subsubsection{N-(tert-Butoxycarbonyl)-N-methyl-N-[2-(1-phenethyl-1H-1,2,3-triazol-4- yl)ethyl] amine 20}

It was prepared as described for 17. From compound 12 (876 mg, $2.77 \mathrm{mmol}), \mathrm{NaH}$ (60\% supension in mineral oil, $167 \mathrm{mg}, 4.17 \mathrm{mmol})$, and iodomethane $(0.19 \mathrm{~mL}, 433$ $\mathrm{mg}, 3.05 \mathrm{mmol}$ ), stirring at $\mathrm{rt}$ for $1.5 \mathrm{~h}$, compound 20 (738 $\mathrm{mg}, 81 \%$ yield) was obtained as a yellowish oil; $R_{f} 0.57\left(\mathrm{CH}_{2} \mathrm{Cl}_{2} / \mathrm{MeOH} / 50 \%\right.$ aq. $\mathrm{NH}_{4} \mathrm{OH}$ 9.5:0.5:0.05); mp 58-60 ${ }^{\circ} \mathrm{C}$; IR (ATR) $v 1686(\mathrm{C}=\mathrm{O}) \mathrm{cm}^{-1} ;{ }^{1} \mathrm{H} \mathrm{NMR}\left(400 \mathrm{MHz}, \mathrm{CDCl}_{3}\right) \delta 1.42\left[\mathrm{~s}, 9 \mathrm{H}, \mathrm{C}\left(\mathrm{CH}_{3}\right)_{3}\right]$, $2.80\left(\mathrm{~s}, 3 \mathrm{H}, \mathrm{N}-\mathrm{CH}_{3}\right), 2.90\left(\mathrm{~m}, 2 \mathrm{H}, 4-\mathrm{CH}_{2}-\mathrm{CH}_{2}-\mathrm{N}\right), 3.18$ (t, $J=7.2 \mathrm{~Hz}, 2 \mathrm{H}$, $\left.1-\mathrm{CH}_{2}-\mathrm{CH}_{2}-\mathrm{Ph}\right), 3.48\left(\mathrm{~m}, 2 \mathrm{H}, 4-\mathrm{CH}_{2}-\mathrm{CH}_{2}-\mathrm{N}\right), 4.54$ (t, $J=7.2 \mathrm{~Hz}, 2 \mathrm{H}$, $1-\mathrm{CH}_{2}-\mathrm{CH}_{2}-\mathrm{Ph}$ ), 7.03 (br s), 7.19 (br s) $(5-\mathrm{H}), 7.10[\mathrm{dm}, J=8.0 \mathrm{~Hz}, 2 \mathrm{H}$, $\mathrm{Ph}-\mathrm{C} 2(6)-H], 7.21-7.32$ (complex signal, 3H, $\mathrm{Ph}-\mathrm{C} 3(5)-H, \mathrm{Ph}-\mathrm{C} 4-H]$; ${ }^{13} \mathrm{C} \mathrm{NMR}$

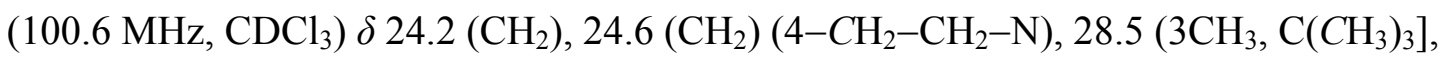
$34.5\left(\mathrm{CH}_{3}, \quad \mathrm{~N}-\mathrm{CH}_{3}\right), \quad 36.9\left(\mathrm{CH}_{2}, \quad 1-\mathrm{CH}_{2}-\mathrm{CH}_{2}-\mathrm{Ph}\right), \quad 48.1 \quad\left(\mathrm{CH}_{2}\right), \quad 48.9 \quad\left(\mathrm{CH}_{2}\right)$ (4- $\left.\mathrm{CH}_{2}-\mathrm{CH}_{2}-\mathrm{N}\right), 51.6\left(\mathrm{CH}_{2}, 1-\mathrm{CH}_{2}-\mathrm{CH}_{2}-\mathrm{Ph}\right), 79.5\left[\mathrm{C}, \mathrm{C}\left(\mathrm{CH}_{3}\right)_{3}\right], 121.8(\mathrm{CH}, \mathrm{C} 5)$, $127.1(\mathrm{CH}), 128.7(2 \mathrm{CH}), 128.9(2 \mathrm{CH})(\mathrm{Ph}-\mathrm{CH}), 137.2$ (C, Ph-C1), 144.8 (br C, C4), 155.8 (C, NCOO); HRMS (ESI), calcd for $\left(\mathrm{C}_{18} \mathrm{H}_{26} \mathrm{~N}_{4} \mathrm{O}_{2}+\mathrm{H}^{+}\right)$331.2129, found 331.2122 .

\subsubsection{3. $N$-(tert-Butoxycarbonyl)-N-methyl-N-[(1-methyl-1H-1,2,3-triazol-4- yl)methyl] amine 21}

It was prepared as described for 17. From compound $\mathbf{1 3}$ (928 mg, $4.38 \mathrm{mmol}), \mathrm{NaH}$ (60\% supension in mineral oil, $263 \mathrm{mg}, 6.57 \mathrm{mmol})$, and iodomethane $(0.30 \mathrm{~mL}, 684$ $\mathrm{mg}, 4.82 \mathrm{mmol})$, stirring at $\mathrm{rt}$ for $1.5 \mathrm{~h}$, compound 21 (945 mg, 95\% yield) was obtained as a yellowish oil; $R_{f} 0.31\left(\mathrm{CH}_{2} \mathrm{Cl}_{2} / \mathrm{MeOH} / 50 \%\right.$ aq. $\mathrm{NH}_{4} \mathrm{OH}$ 9.5:0.5:0.05); IR (ATR)

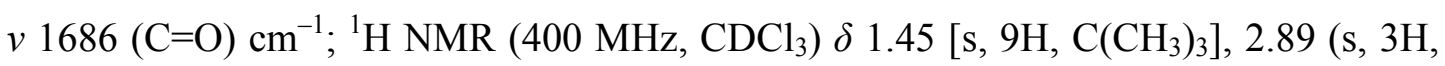
$\left.\mathrm{N}-\mathrm{CH}_{3}\right), 4.07\left(\mathrm{~s}, 3 \mathrm{H}, 1-\mathrm{CH}_{3}\right), 4.47\left(\mathrm{~s}, 2 \mathrm{H}, 4-\mathrm{CH}_{2}-\mathrm{N}\right), 7.37$ (br s), $7.49($ broad s) $(5-\mathrm{H})$; 
${ }^{13} \mathrm{C} \mathrm{NMR}\left(100.6 \mathrm{MHz}, \mathrm{CDCl}_{3}\right) \delta 28.6\left[3 \mathrm{CH}_{3}, \mathrm{C}\left(\mathrm{CH}_{3}\right)_{3}\right], 34.2\left(\mathrm{br} \mathrm{CH}_{3}\right), 34.6\left(\mathrm{br} \mathrm{CH}_{3}\right)$ $\left(\mathrm{N}-\mathrm{CH}_{3}\right), 36.8\left(\mathrm{CH}_{3}, 1-\mathrm{CH}_{3}\right), 43.8\left(\right.$ br $\left.\mathrm{CH}_{2}\right), 44.5\left(\right.$ br $\left.\mathrm{CH}_{2}\right)\left(4-\mathrm{CH}_{2}-\mathrm{N}\right), 79.9$ [br C, $C\left(\mathrm{CH}_{3}\right)_{3}$ ], 122.6 (br CH), 123.5 (br CH) (C5), 145.3 (br C), 145.8 (br C) (C4), 155.3 (br C), 155.9 (br C) (NCOO); HRMS (ESI), calcd for $\left(\mathrm{C}_{10} \mathrm{H}_{18} \mathrm{~N}_{4} \mathrm{O}_{2}+\mathrm{Na}^{+}\right)$249.1322; found 249.1323 .

\subsubsection{N-(tert-Butoxycarbonyl)-N-methyl-N-[2-(1-phenyl-1H-1,2,3-triazol-4-} yl)ethyl]amine 22

It was prepared as described for 17. From compound 14 (1.82 g, $6.32 \mathrm{mmol}), \mathrm{NaH}$ (60\% supension in mineral oil, $0.76 \mathrm{~g}, 19.0 \mathrm{mmol})$, and iodomethane $(1.96 \mathrm{~mL}, 4.47 \mathrm{~g}$, $31.5 \mathrm{mmol}$ ), stirring at rt overnight, compound 22 (1.82 g, 95\% yield) was obtained as a yellowish oil; $R_{f} 0.86\left(\mathrm{CH}_{2} \mathrm{Cl}_{2} / \mathrm{MeOH} / 50 \%\right.$ aq. $\mathrm{NH}_{4} \mathrm{OH}$ 9:1:0.05); IR (ATR) $v 1689$, $1681(\mathrm{C}=\mathrm{O}) \mathrm{cm}^{-1} ;{ }^{1} \mathrm{H}$ NMR $\left(400 \mathrm{MHz}, \mathrm{CDCl}_{3}\right) \delta 1.42\left[\mathrm{~s}, 9 \mathrm{H}, \mathrm{C}\left(\mathrm{CH}_{3}\right)_{3}\right], 2.88(\mathrm{~s}, 3 \mathrm{H}$, $\left.\mathrm{N}-\mathrm{CH}_{3}\right), 3.04\left(\mathrm{~m}, 2 \mathrm{H}, 4-\mathrm{CH}_{2}-\mathrm{CH}_{2}-\mathrm{N}\right), 3.61\left(\mathrm{~m}, 2 \mathrm{H}, 4-\mathrm{CH}_{2}-\mathrm{CH}_{2}-\mathrm{N}\right), 7.43(\mathrm{~m}, 1 \mathrm{H}$, $\mathrm{Ph}-\mathrm{C} 4-H), 7.51[\mathrm{~m}, 2 \mathrm{H}, \mathrm{Ph}-\mathrm{C} 3(5)-H], 7.71[\mathrm{dm}, J=8.8 \mathrm{~Hz}, 2 \mathrm{H}, \mathrm{Ph}-\mathrm{C} 2(6)-H], 7.89$ (br s, $1 \mathrm{H}, 5-\mathrm{H}) ;{ }^{13} \mathrm{C}$ NMR $\left(100.6 \mathrm{MHz}, \mathrm{CDCl}_{3}\right) \delta 24.3\left(\right.$ br $\left.\mathrm{CH}_{2}\right), 24.6\left(\right.$ br $\left.\mathrm{CH}_{2}\right)$ $\left(4-\mathrm{CH}_{2}-\mathrm{CH}_{2}-\mathrm{N}\right), 28.5\left[3 \mathrm{CH}_{3}, \mathrm{C}\left(\mathrm{CH}_{3}\right)_{3}\right], 34.4\left(\mathrm{CH}_{3}, \mathrm{~N}-\mathrm{CH}_{3}\right), 47.8\left(\right.$ br $\left.\mathrm{CH}_{2}\right), 48.7$ (br $\left.\mathrm{CH}_{2}\right)\left(4-\mathrm{CH}_{2}-\mathrm{CH}_{2}-\mathrm{N}\right), 79.6\left[\mathrm{C}, \mathrm{C}\left(\mathrm{CH}_{3}\right)_{3}\right], 119.8(\mathrm{CH}, \mathrm{Ph}-\mathrm{C} 4), 120.5(\mathrm{CH}, \mathrm{C} 5), 128.6$ (2CH), 129.8 (2CH) [Ph-C2(6), Ph-C3(5)], 137.2 (C, Ph-C1), 145.9 (br C, C4), 155.8 (br C, NCOO); HRMS (ESI), calcd for $\left(\mathrm{C}_{16} \mathrm{H}_{22} \mathrm{~N}_{4} \mathrm{O}_{2}+\mathrm{H}^{+}\right)$303.1816, found 303.1809.

4.1.15. N-[(1-Benzyl-1H-1,2,3-triazol-4-yl)methyl]-N-(tert-butoxycarbonyl)-Nmethylamine 23

It was prepared as described for 17. From compound 15 (1.60 g, $5.16 \mathrm{mmol}), \mathrm{NaH}$ (60\% suspension in mineral oil, $670 \mathrm{mg}, 16.7 \mathrm{mmol})$, and iodomethane $(1.73 \mathrm{~mL}, 3.94$ g, $27.8 \mathrm{mmol}$ ), stirring at $\mathrm{rt}$ overnight, compound 23 (1.30 g, 83\% yield) was obtained as a brown solid; $R_{f} 0.68\left(\mathrm{CH}_{2} \mathrm{Cl}_{2} / \mathrm{MeOH} / \mathrm{NH}_{4} \mathrm{OH}\right.$ 9.75:0.25:0.05); mp 79-81 ${ }^{\circ} \mathrm{C}$; IR (ATR) $v 1679(\mathrm{C}=\mathrm{O}) \mathrm{cm}^{-1} ;{ }^{1} \mathrm{H}$ NMR $\left(400 \mathrm{MHz}, \mathrm{CDCl}_{3}\right) \delta 1.41\left[\mathrm{br} \mathrm{s}, 9 \mathrm{H}, \mathrm{C}\left(\mathrm{CH}_{3}\right)_{3}\right]$, $2.89\left(\mathrm{~s}, 3 \mathrm{H}, \mathrm{N}-\mathrm{CH}_{3}\right), 4.45\left(\mathrm{~s}, 2 \mathrm{H}, 4-\mathrm{CH}_{2}-\mathrm{N}\right), 5.50\left(\mathrm{~s}, 2 \mathrm{H}, 1-\mathrm{CH}_{2}-\mathrm{Ph}\right), 7.26$ [br dd, $J=$ $\left.8.0 \mathrm{~Hz}, J^{\prime}=1.6 \mathrm{~Hz}, 2 \mathrm{H}, \mathrm{Ph}-\mathrm{C} 2(6)-H\right]$ ] 7.34-7.40 [complex signal, $3 \mathrm{H}, \mathrm{Ph}-\mathrm{C} 3(5)-H$, $\mathrm{Ph}-\mathrm{C} 4-H], 7.44$ (br s, $1 \mathrm{H}, 5-\mathrm{H}) ;{ }^{13} \mathrm{C}$ NMR $\left(100.6 \mathrm{MHz}, \mathrm{CDCl}_{3}\right) \delta 28.4\left[3 \mathrm{CH}_{3}\right.$, $\left.\mathrm{C}\left(\mathrm{CH}_{3}\right)_{3}\right], 34.5\left(\mathrm{CH}_{3}, \mathrm{~N}-\mathrm{CH}_{3}\right), 43.8\left(\mathrm{CH}_{2}\right), 44.6\left(\mathrm{CH}_{2}\right)\left(4-\mathrm{CH}_{2}-\mathrm{N}\right), 54.2\left(\mathrm{CH}_{2}\right.$, $\left.1-\mathrm{CH}_{2}-\mathrm{Ph}\right), 79.8\left[\mathrm{C}, \mathrm{C}\left(\mathrm{CH}_{3}\right)_{3}\right], 121.6(\mathrm{CH}), 122.4(\mathrm{CH})(\mathrm{C} 5), 128.1(2 \mathrm{CH}), 128.8$ 
(CH), 129.2 (2CH) (Ph-CH), $134.7(\mathrm{C}, \mathrm{Ph}-\mathrm{C1}), 145.4$ (C), $145.6(\mathrm{C})(\mathrm{C} 4), 155.4(\mathrm{C})$, 155.8 (C) (NCOO); HRMS (ESI), calcd for $\left(\mathrm{C}_{16} \mathrm{H}_{22} \mathrm{~N}_{4} \mathrm{O}_{2}+\mathrm{Na}^{+}\right) 325.1635$, found 325.1638 .

4.1.16. N-(tert-Butoxycarbonyl)-N-methyl-N-[(1-phenetyl-1H-1,2,3-triazol-4yl)methyl] amine 24

It was prepared as described for 17. From compound 16 (1.27 g, $4.20 \mathrm{mmol}), \mathrm{NaH}$ (60\% suspension in mineral oil, $505 \mathrm{mg}, 12.6 \mathrm{mmol})$, and iodomethane (1.31 mL, 2.99 $\mathrm{g}, 21.0 \mathrm{mmol})$, stirring at rt overnight, compound 24 (1.07 g, 81\% yield) was obtained as a brown oil; $R_{f} 0.59\left(\mathrm{CH}_{2} \mathrm{Cl}_{2} / \mathrm{MeOH} / \mathrm{NH}_{4} \mathrm{OH}\right.$ 9.75:0.25:0.05); IR (ATR) $v 1687$ $(\mathrm{C}=\mathrm{O}) \mathrm{cm}^{-1} ;{ }^{1} \mathrm{H}$ NMR $\left(400 \mathrm{MHz}, \mathrm{CDCl}_{3}\right) \delta 1.43\left[\mathrm{~s}, 9 \mathrm{H}, \mathrm{C}\left(\mathrm{CH}_{3}\right)_{3}\right], 2.84(\mathrm{~s}, 3 \mathrm{H}$, $\left.\mathrm{N}-\mathrm{CH}_{3}\right), 3.19$ (t, $\left.J=7.2 \mathrm{~Hz}, 2 \mathrm{H}, 1-\mathrm{CH}_{2}-\mathrm{CH}_{2}-\mathrm{Ph}\right), 4.43\left(\mathrm{~s}, 2 \mathrm{H}, 4-\mathrm{CH}_{2}-\mathrm{N}\right), 4.56(\mathrm{~m}$, $2 \mathrm{H}, 1-\mathrm{CH}_{2}-\mathrm{CH}_{2}-\mathrm{Ph}$ ), 7.08 [dm, $\left.J=8.4 \mathrm{~Hz}, 2 \mathrm{H}, \mathrm{Ph}-\mathrm{C} 2(6)-H\right], 7.20-7.32$ [complex signal, 4H, 5-H, Ph-C3(5)-H, Ph-C4-H]; $\left.{ }^{13} \mathrm{C} \mathrm{NMR} \mathrm{(100.6} \mathrm{MHz,} \mathrm{CDCl}_{3}\right) \delta 28.2\left[\mathrm{CH}_{3}\right.$, $\mathrm{C}\left(\mathrm{CH}_{3}\right)_{3}$ minor rotamer], $28.3\left[\mathrm{CH}_{3}, \mathrm{C}\left(\mathrm{CH}_{3}\right)_{3}\right.$ major rotamer], $33.9\left(\mathrm{CH}_{3}, \mathrm{~N}-\mathrm{CH}_{3}\right.$ minor rotamer), $34.2\left(\mathrm{CH}_{3}, \mathrm{~N}-\mathrm{CH}_{3}\right.$ major rotamer), $36.5\left(\mathrm{CH}_{2}, 1-\mathrm{CH}_{2}-\mathrm{CH}_{2}-\mathrm{Ph}\right.$ minor rotamer), $36.6\left(\mathrm{CH}_{2}, 1-\mathrm{CH}_{2}-\mathrm{CH}_{2}-\mathrm{Ph}\right.$ major rotamer), $43.5\left(\mathrm{CH}_{2}, 4-\mathrm{CH}_{2}-\mathrm{N}\right.$ major rotamer), $44.2\left(\mathrm{CH}_{2}, 4-\mathrm{CH}_{2}-\mathrm{N}\right.$ minor rotamer), $51.4\left(\mathrm{CH}_{2}, 1-\mathrm{CH}_{2}-\mathrm{CH}_{2}-\mathrm{Ph}\right.$ minor rotamer), $51.5\left(\mathrm{CH}_{2}, 1-\mathrm{CH}_{2}-\mathrm{CH}_{2}-\mathrm{Ph}\right.$ major rotamer), $79.6\left[\mathrm{br} \mathrm{C}, \mathrm{C}\left(\mathrm{CH}_{3}\right)_{3}\right], 121.9(\mathrm{CH}$, C5 minor rotamer), 122.6 ( $\mathrm{CH}, \mathrm{C} 5$ major rotamer), $126.8(\mathrm{CH}, \mathrm{Ph}-\mathrm{C} 4$ minor rotamer), $127.0(\mathrm{CH}, \mathrm{Ph}-\mathrm{C} 4$ major rotamer), $128.4(\mathrm{CH}), 128.5(\mathrm{CH}), 128.7(\mathrm{CH})[\mathrm{Ph}-\mathrm{C} 2(6)$, $\mathrm{Ph}-C 3(5)], 136.8$ (C, Ph-C1 minor rotamer), 136.9 (C, Ph-C1 major rotamer), 144.4 (br C, C4), 155.2 (C, NCOO minor rotamer), 155.7 (C, NCOO major rotamer); HRMS (ESI), calcd for $\left(\mathrm{C}_{17} \mathrm{H}_{24} \mathrm{~N}_{4} \mathrm{O}_{2}+\mathrm{H}^{+}\right) 317.1972$, found 317.1979 .

\subsubsection{N-[(1-Ethyl-1H-1,2,3-triazol-4-yl)methyl]-N-methylamine 25}

To a solution of $17(411 \mathrm{mg}, 1.71 \mathrm{mmol})$ in $\mathrm{CH}_{2} \mathrm{Cl}_{2}(6 \mathrm{~mL}), \mathrm{H}_{3} \mathrm{PO}_{4}(85 \%$ purity, 2.96 $\mathrm{mL}, 25.7 \mathrm{mmol}$ ) was added. The reaction mixture was stirred at $\mathrm{rt}$ for $2 \mathrm{~h}$, cooled to 0 ${ }^{\circ} \mathrm{C}$, diluted with $\mathrm{H}_{2} \mathrm{O}(15 \mathrm{~mL})$, alkalinized dropwise to $\mathrm{pH}=14$ with $5 \mathrm{~N} \mathrm{NaOH}$, and extracted with $\mathrm{CH}_{2} \mathrm{Cl}_{2}(2 \times 30 \mathrm{~mL})$. The combined organic extracts were dried over anhydrous $\mathrm{Na}_{2} \mathrm{SO}_{4}$ and evaporated under reduced pressure to give amine 25 (205 $\mathrm{mg}$, $86 \%$ yield) as a colorless oil; $R_{f} 0.07\left(\mathrm{CH}_{2} \mathrm{Cl}_{2} / \mathrm{MeOH} / 50 \%\right.$ aq. $\mathrm{NH}_{4} \mathrm{OH}$ 9:1:0.05). 
A solution of amine $25(25 \mathrm{mg}, 0.18 \mathrm{mmol})$ in $\mathrm{CH}_{2} \mathrm{Cl}_{2}(4 \mathrm{~mL})$ was filtered through a PTFE filter $(0.2 \mu \mathrm{m})$, treated with a $\mathrm{HCl} / \mathrm{MeOH}$ solution $(0.75 \mathrm{~N}, 0.71 \mathrm{~mL}, 0.53$ $\mathrm{mmol})$, and evaporated under reduced pressure. The resulting residue was washed with pentane $(3 \times 2 \mathrm{~mL})$ to give, after drying under standard conditions, $25 \cdot \mathrm{HCl}(28 \mathrm{mg})$ as a beige sticky solid; IR (ATR) v 3500-2500 ( $\max$ at 3386, 3126, 3080, 3028, 2981, 2935, 2756, 2695, ${ }^{+} \mathrm{N}-\mathrm{H}$ and $\left.\mathrm{C}-\mathrm{H}\right) \mathrm{cm}^{-1}{ }^{1} \mathrm{H}$ NMR (400 MHz, $\left.\mathrm{CD}_{3} \mathrm{OD}\right) \delta 1.55(\mathrm{t}, J=7.2 \mathrm{~Hz}$, $\left.3 \mathrm{H}, 1-\mathrm{CH}_{2}-\mathrm{CH}_{3}\right), 2.76\left(\mathrm{~s}, 3 \mathrm{H}, \mathrm{N}-\mathrm{CH}_{3}\right), 4.35\left(\mathrm{~s}, 2 \mathrm{H}, 4-\mathrm{CH}_{2}-\mathrm{N}\right), 4.50$ (q, J = $7.2 \mathrm{~Hz}$, $\left.2 \mathrm{H}, 1-\mathrm{CH}_{2}-\mathrm{CH}_{3}\right), 4.96\left(\mathrm{~s},{ }^{+} \mathrm{NH}_{2}\right), 8.22(\mathrm{~s}, 1 \mathrm{H}, 5-\mathrm{H}) ;{ }^{13} \mathrm{C} \mathrm{NMR}\left(100.6 \mathrm{MHz}, \mathrm{CD}_{3} \mathrm{OD}\right) \delta$ $15.8\left(\mathrm{CH}_{3}, 1-\mathrm{CH}_{2}-\mathrm{CH}_{3}\right), 33.1\left(\mathrm{CH}_{3}, \mathrm{~N}-\mathrm{CH}_{3}\right), 44.2\left(\mathrm{CH}_{2}, 4-\mathrm{CH}_{2}-\mathrm{N}\right), 46.8\left(\mathrm{CH}_{2}\right.$, $\left.1-\mathrm{CH}_{2}-\mathrm{CH}_{3}\right), 126.2(\mathrm{CH}, \mathrm{C} 5), 139.3(\mathrm{C}, \mathrm{C} 4)$; HRMS (ESI), calcd for $\left(\mathrm{C}_{6} \mathrm{H}_{12} \mathrm{~N}_{4}+\mathrm{H}^{+}\right)$ 141.1135, found 141.1133 .

\subsubsection{8. $N$-[2-(1-Ethyl-1H-1,2,3-triazol-4-yl)ethyl]-N-methylamine 26}

It was prepared as described for 25 . From 18 (263 mg, $1.03 \mathrm{mmol})$ and $\mathrm{H}_{3} \mathrm{PO}_{4}(85 \%$ purity, $1.80 \mathrm{~mL}, 15.6 \mathrm{mmol}$ ), and stirring at $\mathrm{rt}$ for $4 \mathrm{~h}$, amine $\mathbf{2 6}$ (143 $\mathrm{mg}, 90 \%$ yield) was obtained as a colorless oil; $R_{f} 0.07\left(\mathrm{CH}_{2} \mathrm{Cl}_{2} / \mathrm{MeOH} / 50 \%\right.$ aq. $\mathrm{NH}_{4} \mathrm{OH}$ 9:1:0.05).

26. $\mathrm{HCl}$ : beige sticky solid, IR (ATR) v 3500-2400 (max at 3395, 3116, 3064, 2976, 2936, 2752, 2691, 2446, ${ }^{+} \mathrm{N}-\mathrm{H}$ and C-H) $\mathrm{cm}^{-1}$; ${ }^{1} \mathrm{H}$ NMR (400 MHz, $\left.\mathrm{CD}_{3} \mathrm{OD}\right) \delta 1.54$ (t, $\left.J=7.2 \mathrm{~Hz}, 3 \mathrm{H}, 1-\mathrm{CH}_{2}-\mathrm{CH}_{3}\right), 2.76\left(\mathrm{~s}, 3 \mathrm{H}, \mathrm{N}-\mathrm{CH}_{3}\right), 3.17$ (t, $J=7.2 \mathrm{~Hz}, 2 \mathrm{H}$, $4-\mathrm{CH}_{2}-\mathrm{CH}_{2}-\mathrm{N}$ ), 3.37 (t, $J=7.2 \mathrm{~Hz}, 2 \mathrm{H}, 4-\mathrm{CH}_{2}-\mathrm{CH}_{2}-\mathrm{N}$ ), 4.48 (q, $J=7.2 \mathrm{~Hz}, 2 \mathrm{H}$, $\left.1-\mathrm{CH}_{2}-\mathrm{CH}_{3}\right), 4.87\left(\mathrm{~s},{ }^{+} \mathrm{NH}_{2}\right), 8.07(\mathrm{~s}, 1 \mathrm{H}, 5-\mathrm{H}) ;{ }^{13} \mathrm{C} \mathrm{NMR}\left(100.6 \mathrm{MHz}, \mathrm{CD}_{3} \mathrm{OD}\right) \delta 15.6$ $\left(\mathrm{CH}_{3}, 1-\mathrm{CH}_{2}-\mathrm{CH}_{3}\right), 22.9\left(\mathrm{CH}_{2}, 4-\mathrm{CH}_{2}-\mathrm{CH}_{2}-\mathrm{N}\right), 33.7\left(\mathrm{CH}_{3}, \mathrm{~N}-\mathrm{CH}_{3}\right), 47.1\left(\mathrm{CH}_{2}\right.$, 1- $\left.\mathrm{CH}_{2}-\mathrm{CH}_{3}\right), 49.3\left(\mathrm{CH}_{2}, 4-\mathrm{CH}_{2}-\mathrm{CH}_{2}-\mathrm{N}\right), 124.6(\mathrm{CH}, \mathrm{C} 5), 143.3$ (C, C4); HRMS (ESI), calcd for $\left(\mathrm{C}_{7} \mathrm{H}_{14} \mathrm{~N}_{4}+\mathrm{H}^{+}\right)$155.1291, found 155.1297.

\subsubsection{N-[2-(1-Benzyl-1H-1,2,3-triazol-4-yl)ethyl]-N-methylamine 27}

It was prepared as described for 25. From 19 (1.12 g, $3.54 \mathrm{mmol})$ and $\mathrm{H}_{3} \mathrm{PO}_{4}(85 \%$ purity, $6.08 \mathrm{~mL}, 52.7 \mathrm{mmol}$ ), amine $27(721 \mathrm{mg}$, 94\% yield) was obtained as a yellow oil; $R_{f} 0.23\left(\mathrm{CH}_{2} \mathrm{Cl}_{2} / \mathrm{MeOH} / 50 \%\right.$ aq. $\mathrm{NH}_{4} \mathrm{OH}$ 9.5:0.5:0.05).

27. $\mathrm{HCl}$ : beige solid, $\mathrm{mp} 189-190^{\circ} \mathrm{C}$; IR (ATR) v 3600-2400 (max at 3554, 3064, 3013, 2927, 2868, 2735, 2685, 2447, C-H, ${ }^{+} \mathrm{N}-\mathrm{H}$ and $\left.\mathrm{C}-\mathrm{H}\right) \mathrm{cm}^{-1} ;{ }^{1} \mathrm{H}$ NMR (400 MHz, $\left.\mathrm{CD}_{3} \mathrm{OD}\right) \delta 2.75\left(\mathrm{~s}, 3 \mathrm{H}, \mathrm{N}-\mathrm{CH}_{3}\right), 3.24\left(\mathrm{t}, J=7.2 \mathrm{~Hz}, 2 \mathrm{H}, 4-\mathrm{CH}_{2}-\mathrm{CH}_{2}-\mathrm{N}\right), 3.38(\mathrm{t}, J=$ $\left.7.2 \mathrm{~Hz}, 2 \mathrm{H}, 4-\mathrm{CH}_{2}-\mathrm{CH}_{2}-\mathrm{N}\right), 4.98\left(\mathrm{~s},{ }^{+} \mathrm{NH}_{2}\right), 5.72$ (s, 2H, 1- $\left.\mathrm{CH}_{2}-\mathrm{Ph}\right), 7.35-7.46$ 
(complex signal, 5H, Ph-CH), $8.30(\mathrm{~s}, 1 \mathrm{H}, 5-H) ;{ }^{13} \mathrm{C} \mathrm{NMR}\left(100.6 \mathrm{MHz}, \mathrm{CD}_{3} \mathrm{OD}\right) \delta$ $22.4\left(\mathrm{CH}_{2}, 4-\mathrm{CH}_{2}-\mathrm{CH}_{2}-\mathrm{N}\right), 33.7\left(\mathrm{CH}_{3}, \mathrm{~N}-\mathrm{CH}_{3}\right), 48.6\left(\mathrm{CH}_{2}, 4-\mathrm{CH}_{2}-\mathrm{CH}_{2}-\mathrm{N}\right), 56.5$ $\left(\mathrm{CH}_{2}, 1-\mathrm{CH}_{2}-\mathrm{Ph}\right), 126.6(\mathrm{CH}, \mathrm{C} 5), 129.8(2 \mathrm{CH}), 130.15(\mathrm{CH}), 130.20(2 \mathrm{CH})(\mathrm{Ph}-\mathrm{CH})$, $135.3(\mathrm{C}, \mathrm{Ph}-\mathrm{C} 1), 142.6(\mathrm{C}, \mathrm{C} 4)$; HRMS (ESI), calcd for $\left(\mathrm{C}_{12} \mathrm{H}_{16} \mathrm{~N}_{4}+\mathrm{H}^{+}\right)$217.1448, found 217.1442 .

\subsubsection{N-Methyl-N-[2-(1-phenethyl-1H-1,2,3-triazol-4-yl)ethyl]amine $\mathbf{2 8}$}

It was prepared as described for 25. From $20(693 \mathrm{mg}, 2.10 \mathrm{mmol})$ and $\mathrm{H}_{3} \mathrm{PO}_{4}(85 \%$ purity, $3.61 \mathrm{~mL}, 31.3 \mathrm{mmol}$ ), amine 28 (472 $\mathrm{mg}$, 98\% yield) was obtained as a yellow oil; $R_{f} 0.26\left(\mathrm{CH}_{2} \mathrm{Cl}_{2} / \mathrm{MeOH} / 50 \%\right.$ aq. $\mathrm{NH}_{4} \mathrm{OH}$ 9.5:0.5:0.05).

28 $\cdot \mathrm{HCl}$ : beige solid, $\mathrm{mp} 160-162^{\circ} \mathrm{C}$; IR (ATR) v 3500-2400 (max at 3413, 3354, 3126, 3092, 2954, 2783, 2703, 2444, ${ }^{+} \mathrm{N}-\mathrm{H}$ and $\left.\mathrm{C}-\mathrm{H}\right) \mathrm{cm}^{-1} ;{ }^{1} \mathrm{H}$ NMR (400 MHz, $\left.\mathrm{CD}_{3} \mathrm{OD}\right) \delta$ $2.75\left(\mathrm{~s}, 3 \mathrm{H}, \mathrm{N}-\mathrm{CH}_{3}\right), 3.25\left(\mathrm{t}, J=7.2 \mathrm{~Hz}, 2 \mathrm{H}, 1-\mathrm{CH}_{2}-\mathrm{CH}_{2}-\mathrm{Ph}\right)$, overimposed in part 3.29 (t, $\left.J=7.2 \mathrm{~Hz}, 2 \mathrm{H}, 4-\mathrm{CH}_{2}-\mathrm{CH}_{2}-\mathrm{N}\right), 3.37$ (t, $\left.J=7.2 \mathrm{~Hz}, 2 \mathrm{H}, 4-\mathrm{CH}_{2}-\mathrm{CH}_{2}-\mathrm{N}\right), 4.80$ (t, $J=7.2 \mathrm{~Hz}, 2 \mathrm{H}, 1-\mathrm{CH}_{2}-\mathrm{CH}_{2}-\mathrm{Ph}$ ), $4.99\left(\mathrm{~s},{ }^{+} \mathrm{NH}_{2}\right.$ ), $7.18-7.30$ (complex signal, 5H, $\mathrm{Ph}-\mathrm{CH}), 8.30(\mathrm{~s}, \quad 1 \mathrm{H}, \quad 5-\mathrm{H}) ;{ }^{13} \mathrm{C}$ NMR (100.6 MHz, CD $\left.{ }_{3} \mathrm{OD}\right) \delta 22.1 \quad\left(\mathrm{CH}_{2}\right.$, $\left.4-\mathrm{CH}_{2}-\mathrm{CH}_{2}-\mathrm{N}\right), 33.8\left(\mathrm{CH}_{3}, \mathrm{~N}-\mathrm{CH}_{3}\right), 36.7\left(\mathrm{CH}_{2}, 1-\mathrm{CH}_{2}-\mathrm{CH}_{2}-\mathrm{Ph}\right), 48.4\left(\mathrm{CH}_{2}\right.$, 4- $\left.\mathrm{CH}_{2}-\mathrm{CH}_{2}-\mathrm{N}\right), 54.6\left(\mathrm{CH}_{2}, 1-\mathrm{CH}_{2}-\mathrm{CH}_{2}-\mathrm{Ph}\right), 127.5(\mathrm{CH}, \mathrm{C} 5), 128.2(\mathrm{CH}), 129.8$ (4CH) $(\mathrm{Ph}-\mathrm{CH}), 137.9(\mathrm{C}, \mathrm{Ph}-\mathrm{C1}), 141.6(\mathrm{C}, \mathrm{C} 4)$; HRMS (ESI), calcd for $\left(\mathrm{C}_{13} \mathrm{H}_{18} \mathrm{~N}_{4}+\right.$ $\left.\mathrm{H}^{+}\right)$231.1604; found 231.1604.

\subsubsection{N-[(1-Benzyl-1H-1,2,3-triazol-4-yl)methyl]-N-methylamine 29}

It was prepared as described for 25 . From $23(723 \mathrm{mg}, 2.39 \mathrm{mmol})$ and $\mathrm{H}_{3} \mathrm{PO}_{4}(85 \%$ purity, $4.14 \mathrm{~mL}, 35.9 \mathrm{mmol}$ ), amine 29 (420 mg, 87\% yield) was obtained as a brown oil; $R_{f} 0.14\left(\mathrm{CH}_{2} \mathrm{Cl}_{2} / \mathrm{MeOH} / 50 \%\right.$ aq. $\mathrm{NH}_{4} \mathrm{OH}$ 9.75:0.25:0.05).

29. $\mathrm{HCl}$ : yellow solid, mp $218-219{ }^{\circ} \mathrm{C}$; IR (ATR) v 3500-2400 (max at 3126, 3059, 3018, 2981, 2940, 2852, 2770, 2697, ${ }^{+} \mathrm{N}-\mathrm{H}$ and $\left.\mathrm{C}-\mathrm{H}\right) \mathrm{cm}^{-1} ;{ }^{1} \mathrm{H}$ NMR (400 MHz, $\left.\mathrm{CD}_{3} \mathrm{OD}\right) \delta 2.74\left(\mathrm{~s}, 3 \mathrm{H}, \mathrm{N}-\mathrm{CH}_{3}\right), 4.33\left(\mathrm{~s}, 2 \mathrm{H}, 4-\mathrm{CH}_{2}-\mathrm{N}\right), 4.87\left(\mathrm{~s},{ }^{+} \mathrm{NH}_{2}\right), 5.64(\mathrm{~s}, 2 \mathrm{H}$, 1- $\mathrm{CH}_{2}-\mathrm{Ph}$ ), 7.31-7.40 (complex signal, $\left.5 \mathrm{H}, \mathrm{Ph}-\mathrm{CH}\right), 8.19$ (s, $\left.1 \mathrm{H}, 5-\mathrm{H}\right) ;{ }^{13} \mathrm{C}$ NMR $\left(100.6 \mathrm{MHz}, \mathrm{CD}_{3} \mathrm{OD}\right) \delta 33.1\left(\mathrm{CH}_{3}, \mathrm{~N}-\mathrm{CH}_{3}\right), 44.2\left(\mathrm{CH}_{2}, 4-\mathrm{CH}_{2}-\mathrm{N}\right), 55.0\left(\mathrm{CH}_{2}\right.$, $\left.1-\mathrm{CH}_{2}-\mathrm{Ph}\right), 126.5(\mathrm{CH}, \mathrm{C} 5), 129.3(2 \mathrm{CH}), 129.7(\mathrm{CH}), 130.0(2 \mathrm{CH})(\mathrm{Ph}-\mathrm{CH}), 136.6$ 
(C, Ph-C1), $139.8(\mathrm{C}, \mathrm{C} 4)$; HRMS (ESI), calcd for $\left(\mathrm{C}_{11} \mathrm{H}_{14} \mathrm{~N}_{4}+\mathrm{H}^{+}\right)$203.1291, found 203.1284 .

\subsubsection{N-Methyl-N-[(1-phenethyl-1H-1,2,3-triazol-4-yl)methyl] amine 30}

It was prepared as described for 25. From $24(983 \mathrm{mg}, 3.11 \mathrm{mmol})$ and $\mathrm{H}_{3} \mathrm{PO}_{4}(85 \%$ purity, $5.37 \mathrm{~mL}, 46.6 \mathrm{mmol}$ ), amine $\mathbf{3 0}$ (258 $\mathrm{mg}$, 38\% yield) was obtained as a colorless oil; $R_{f} 0.16\left(\mathrm{CH}_{2} \mathrm{Cl}_{2} / \mathrm{MeOH} / 50 \%\right.$ aq. $\mathrm{NH}_{4} \mathrm{OH}$ 9.75:0.25:0.05).

30 $\cdot \mathrm{HCl}$ : yellow solid, mp $173-175{ }^{\circ} \mathrm{C}$; IR (ATR) v 3500-2400 (max at 3111, 3069, 3023, 2981, 2925, 2764, 2749, 2692, 2599, 2542, ${ }^{+} \mathrm{N}-\mathrm{H}$ and $\left.\mathrm{C}-\mathrm{H}\right) \mathrm{cm}^{-1} ;{ }^{1} \mathrm{H}$ NMR (400 $\left.\mathrm{MHz}, \mathrm{CD}_{3} \mathrm{OD}\right) \delta 2.67\left(\mathrm{~s}, 3 \mathrm{H}, \mathrm{N}-\mathrm{CH}_{3}\right), 3.24\left(\mathrm{t}, J=7.2 \mathrm{~Hz}, 2 \mathrm{H}, 1 \mathrm{CH}_{2}-\mathrm{CH}_{2}-\mathrm{Ph}\right.$ ), $4.26(\mathrm{~s}$, $\left.2 \mathrm{H}, 4-\mathrm{CH}_{2}-\mathrm{N}\right), 4.71\left(\mathrm{t}, J=7.2 \mathrm{~Hz}, 2 \mathrm{H}, 1-\mathrm{CH}_{2}-\mathrm{CH}_{2}-\mathrm{Ph}\right), 4.87\left(\mathrm{~s},{ }^{+} \mathrm{NH}_{2}\right), 7.15[\mathrm{dm}, J=$ $8.4 \mathrm{~Hz}, 2 \mathrm{H}, \mathrm{Ph}-\mathrm{C} 2(6)-H$ ], 7.16-7.29 [complex signal, 3H, $\mathrm{Ph}-\mathrm{C} 3(5)-H, \mathrm{Ph}-\mathrm{C} 4-H$ ], $7.94(\mathrm{~s}, 1 \mathrm{H}, 5-\mathrm{H}) ;{ }^{13} \mathrm{C} \mathrm{NMR}\left(100.6 \mathrm{MHz}, \mathrm{CD}_{3} \mathrm{OD}\right) \delta 32.9\left(\mathrm{CH}_{3}, \mathrm{~N}-\mathrm{CH}_{3}\right), 37.3\left(\mathrm{CH}_{2}\right.$, $\left.1-\mathrm{CH}_{2}-\mathrm{CH}_{2}-\mathrm{Ph}\right), 44.1\left(\mathrm{CH}_{2}, 4-\mathrm{CH}_{2}-\mathrm{N}\right), 52.7\left(\mathrm{CH}_{2}, 1-\mathrm{CH}_{2}-\mathrm{CH}_{2}-\mathrm{Ph}\right), 126.8(\mathrm{CH}$, C5), $128.0(\mathrm{CH}), 129.7(2 \mathrm{CH}), 129.8(2 \mathrm{CH})(\mathrm{Ph}-\mathrm{CH}), 138.6(\mathrm{C}, \mathrm{Ph}-\mathrm{C} 1), 139.0(\mathrm{C}$, C4); HRMS (ESI), calcd for $\left(\mathrm{C}_{12} \mathrm{H}_{16} \mathrm{~N}_{4}+\mathrm{H}^{+}\right) 217.1448$, found 217.1443.

\subsubsection{3. $N$-[(1-Ethyl-1H-1,2,3-triazol-4-yl)methyl]-N-methyl-N-propargylamine 31}

A solution of amine $25(85 \mathrm{mg}, 0.61 \mathrm{mmol})$ in anhydrous acetone $(20 \mathrm{~mL})$ was cooled to $0{ }^{\circ} \mathrm{C}$ and treated with $\mathrm{Cs}_{2} \mathrm{CO}_{3}(199 \mathrm{mg}, 0.61 \mathrm{mmol})$ and propargyl bromide $(80 \%$ solution in toluene, $0.09 \mathrm{~mL}, 0.61 \mathrm{mmol})$. The reaction mixture was stirred at $\mathrm{rt}$ overnight, then filtered, and evaporated under reduced pressure to give an oily residue (161 mg), which was purified through column chromatography $(40-60 \mu \mathrm{m}$ silica gel, $\mathrm{CH}_{2} \mathrm{Cl}_{2} / \mathrm{MeOH} / 50 \%$ aq. $\mathrm{NH}_{4} \mathrm{OH}$ mixtures, gradient elution). On elution with $\mathrm{CH}_{2} \mathrm{Cl}_{2}$ / $\mathrm{MeOH} / 50 \%$ aq. $\mathrm{NH}_{4} \mathrm{OH}$ 98:2:0.2, propargylamine 31 (97 mg, 89\% yield) was isolated as a colorless oil; $R_{f} 0.33\left(\mathrm{CH}_{2} \mathrm{Cl}_{2} / \mathrm{MeOH} / 50 \%\right.$ aq. $\mathrm{NH}_{4} \mathrm{OH}$ 9:1:0.05).

31· $\mathrm{HCl}$ : beige sticky solid, IR (ATR) v 3500-2300 (max at 3412, 3205, 3126, 2987, 2941, 2568, 2477, 2381, ${ }^{+} \mathrm{N}-\mathrm{H}, \equiv \mathrm{C}-\mathrm{H}$, and $\left.\mathrm{C}-\mathrm{H}\right), 2125(\mathrm{C} \equiv \mathrm{C}) \mathrm{cm}^{-1} ;{ }^{1} \mathrm{H}$ NMR (400 $\left.\mathrm{MHz}, \mathrm{CD}_{3} \mathrm{OD}\right) \delta 1.56\left(\mathrm{t}, J=7.6 \mathrm{~Hz}, 3 \mathrm{H}, 1-\mathrm{CH}_{2}-\mathrm{CH}_{3}\right), 2.94\left(\mathrm{~s}, 3 \mathrm{H}, \mathrm{N}-\mathrm{CH}_{3}\right), 3.39(\mathrm{t}, J$ $=2.4 \mathrm{~Hz}, 1 \mathrm{H}$, propargyl $\mathrm{CH}), 4.12\left(\mathrm{~d}, J=2.4 \mathrm{~Hz}, 2 \mathrm{H}\right.$, propargyl $\left.\mathrm{CH}_{2}\right), 4.50(\mathrm{q}, J=7.6$ $\left.\mathrm{Hz}, 2 \mathrm{H}, 1-\mathrm{CH}_{2}-\mathrm{CH}_{3}\right), 4.54$ (s, 2H, 4- $\left.\mathrm{CH}_{2}-\mathrm{N}\right), 4.87\left(\mathrm{~s},{ }^{+} \mathrm{NH}\right), 8.26(\mathrm{~s}, 1 \mathrm{H}, 5-\mathrm{H}) ;{ }^{13} \mathrm{C}$ NMR (100.6 MHz, CD $\left.{ }_{3} \mathrm{OD}\right) \delta 15.7\left(\mathrm{CH}_{3}, 1-\mathrm{CH}_{2}-\mathrm{CH}_{3}\right), 40.3\left(\mathrm{CH}_{3}, \mathrm{~N}-\mathrm{CH}_{3}\right), 45.6$ $\left(\mathrm{CH}_{2}\right.$, propargyl $\left.\mathrm{CH}_{2}\right), 46.8\left(\mathrm{CH}_{2}, 1-\mathrm{CH}_{2}-\mathrm{CH}_{3}\right), 50.4\left(\mathrm{CH}_{2}, 4-\mathrm{CH}_{2}-\mathrm{N}\right), 73.3(\mathrm{C}$, 
propargyl C), $81.2(\mathrm{CH}$, propargyl $\mathrm{CH}), 127.4(\mathrm{CH}, \mathrm{C} 5), 137.8(\mathrm{C}, \mathrm{C} 4)$; HRMS (ESI), calcd for $\left(\mathrm{C}_{9} \mathrm{H}_{14} \mathrm{~N}_{4}+\mathrm{H}^{+}\right)$179.1291, found 179.1289.

\subsubsection{N-[2-(1-Ethyl-1H-1,2,3-triazol-4-yl)ethyl]-N-methyl-N-propargylamine 32}

It was prepared as described for 31. From amine 26 (299 mg, $1.94 \mathrm{mmol}), \mathrm{Cs}_{2} \mathrm{CO}_{3}(630$ $\mathrm{mg}, 1.93 \mathrm{mmol})$, and propargyl bromide $(80 \%$ solution in toluene, $0.29 \mathrm{~mL}, 1.95$ mmol), and stirring at $0{ }^{\circ} \mathrm{C}$ for $2.5 \mathrm{~h}$, propargylamine 32 (220 mg, 59\% yield) was obtained as a yellow oil, without the need of chromatographic purification; $R_{f} 0.55$ $\left(\mathrm{CH}_{2} \mathrm{Cl}_{2} / \mathrm{MeOH} / 50 \%\right.$ aq. $\mathrm{NH}_{4} \mathrm{OH}$ 9:1:0.05).

32. $\mathrm{HCl}$ : yellow sticky solid, IR (ATR) v 3500-2300 (max at 3417, 3191, 2937, 2521, 2434, 2359, ${ }^{+} \mathrm{NH}, \equiv \mathrm{C}-\mathrm{H}$, and $\left.\mathrm{C}-\mathrm{H}\right), 2121(\mathrm{C} \equiv \mathrm{C}) \mathrm{cm}^{-1} ;{ }^{1} \mathrm{H}$ NMR (400 MHz, $\left.\mathrm{CD}_{3} \mathrm{OD}\right) \delta$ $1.53\left(\mathrm{t}, J=7.2 \mathrm{~Hz}, 3 \mathrm{H}, 1-\mathrm{CH}_{2}-\mathrm{CH}_{3}\right), 3.05\left(\mathrm{~s}, 3 \mathrm{H}, \mathrm{N}-\mathrm{CH}_{3}\right), 3.24(\mathrm{t}, J=7.2 \mathrm{~Hz}, 2 \mathrm{H}$, 4- $\mathrm{CH}_{2}-\mathrm{CH}_{2}-\mathrm{N}$ ), 3.41 (t, $J=2.8 \mathrm{~Hz}, 1 \mathrm{H}$, propargyl $\mathrm{CH}$ ), 3.62 (br t, $J=7.2 \mathrm{~Hz}, 2 \mathrm{H}$, 4- $\left.\mathrm{CH}_{2}-\mathrm{CH}_{2}-\mathrm{N}\right), 4.24\left(\mathrm{~d}, J=2.8 \mathrm{~Hz}, 2 \mathrm{H}\right.$, propargyl $\left.\mathrm{CH}_{2}\right), 4.46(\mathrm{q}, J=7.2 \mathrm{~Hz}, 2 \mathrm{H}$, $\left.1-\mathrm{CH}_{2}-\mathrm{CH}_{3}\right), 4.86\left(\mathrm{~s},{ }^{+} \mathrm{NH}\right), 8.01(\mathrm{~s}, 1 \mathrm{H}, 5-\mathrm{H}) ;{ }^{13} \mathrm{C} \mathrm{NMR}\left(100.6 \mathrm{MHz}, \mathrm{CD}_{3} \mathrm{OD}\right) \delta 15.7$ $\left(\mathrm{CH}_{3}, 1-\mathrm{CH}_{2}-\mathrm{CH}_{3}\right), 21.6\left(\mathrm{CH}_{2}, 4-\mathrm{CH}_{2}-\mathrm{CH}_{2}-\mathrm{N}\right), 40.7\left(\mathrm{CH}_{3}, \mathrm{~N}-\mathrm{CH}_{3}\right), 46.3\left(\mathrm{CH}_{2}\right.$, propargyl $\left.\mathrm{CH}_{2}\right), 46.8\left(\mathrm{CH}_{2}, 1-\mathrm{CH}_{2}-\mathrm{CH}_{3}\right), 55.4\left(\mathrm{CH}_{2}, 4-\mathrm{CH}_{2}-\mathrm{CH}_{2}-\mathrm{N}\right), 72.6(\mathrm{C}$, propargyl C), $81.6(\mathrm{CH}$, propargyl $\mathrm{CH}), 124.2(\mathrm{CH}, \mathrm{C} 5), 143.1(\mathrm{C}, \mathrm{C} 4)$; HRMS (ESI), calcd for $\left(\mathrm{C}_{10} \mathrm{H}_{16} \mathrm{~N}_{4}+\mathrm{H}^{+}\right)$193.1448, found 193.1440.

\subsubsection{5. $N$-[2-(1-Benzyl-1H-1,2,3-triazol-4-yl)ethyl]-N-methyl-N-propargylamine 33}

It was prepared as described for 31. From amine 27 (690 mg, $3.19 \mathrm{mmol}), \mathrm{Cs}_{2} \mathrm{CO}_{3}(1.04$ g, $3.19 \mathrm{mmol}$ ), and propargyl bromide ( $80 \%$ solution in toluene, $0.47 \mathrm{~mL}, 3.16 \mathrm{mmol})$, and stirring at $\mathrm{rt}$ for $2 \mathrm{~h}$, propargylamine $33(577 \mathrm{mg}, 71 \%$ yield) was obtained as a yellow oil, without the need of chromatographic purification; $R_{f} 0.48\left(\mathrm{CH}_{2} \mathrm{Cl}_{2} / \mathrm{MeOH} /\right.$ $50 \%$ aq. $\mathrm{NH}_{4} \mathrm{OH}$ 9.5:0.5:0.05).

33 $\cdot \mathrm{HCl}$ : brown solid, $\mathrm{mp} 97-99{ }^{\circ} \mathrm{C}$; IR (ATR) $v$ 3500-2300 (max at 3413, 3194, 2948, 2522, ${ }^{+} \mathrm{N}-\mathrm{H}, \equiv \mathrm{C}-\mathrm{H}$, and $\left.\mathrm{C}-\mathrm{H}\right), 2124(\mathrm{C} \equiv \mathrm{C}) \mathrm{cm}^{-1} ;{ }^{1} \mathrm{H}$ NMR (400 MHz, CD $\left.{ }_{3} \mathrm{OD}\right) \delta 3.03$ $\left(\mathrm{s}, 3 \mathrm{H}, \mathrm{N}-\mathrm{CH}_{3}\right), 3.31$ (t, $\left.J=7.2 \mathrm{~Hz}, 2 \mathrm{H}, 4-\mathrm{CH}_{2}-\mathrm{CH}_{2}-\mathrm{N}\right), 3.39$ (t, $J=2.4 \mathrm{~Hz}, 1 \mathrm{H}$, propargyl $\mathrm{CH}), 3.62\left(\mathrm{~m}, 2 \mathrm{H}, 4-\mathrm{CH}_{2}-\mathrm{CH}_{2}-\mathrm{N}\right), 4.23\left(\mathrm{~d}, J=2.4 \mathrm{~Hz}, 2 \mathrm{H}\right.$, propargyl $\left.\mathrm{CH}_{2}\right)$, $5.00\left(\mathrm{~s},{ }^{+} \mathrm{NH}\right), 5.68\left(\mathrm{~s}, 2 \mathrm{H}, 1-\mathrm{CH}_{2}-\mathrm{Ph}\right), 7.33-7.43$ (complex signal, 5H, $\left.\mathrm{Ph}-\mathrm{CH}\right), 8.23$ (s, 1H, 5-H); ${ }^{13} \mathrm{C}$ NMR (100.6 MHz, $\left.\mathrm{CD}_{3} \mathrm{OD}\right) \delta 21.2\left(\mathrm{CH}_{2}, 4-\mathrm{CH}_{2}-\mathrm{CH}_{2}-\mathrm{N}\right), 40.7\left(\mathrm{CH}_{3}\right.$, $\left.\mathrm{N}-\mathrm{CH}_{3}\right), 46.4\left(\mathrm{CH}_{2}\right.$, propargyl $\left.\mathrm{CH}_{2}\right), 54.8\left(\mathrm{CH}_{2}, 4-\mathrm{CH}_{2}-\mathrm{CH}_{2}-\mathrm{N}\right), 56.1\left(\mathrm{CH}_{2}\right.$, 
1- $\left.\mathrm{CH}_{2}-\mathrm{Ph}\right), 72.6(\mathrm{C}$, propargyl C), $81.7(\mathrm{CH}$, propargyl $\mathrm{CH}), 126.1(\mathrm{CH}, \mathrm{C} 5), 129.6$ $(2 \mathrm{CH}), 130.0(\mathrm{CH}), 130.1(2 \mathrm{CH})(\mathrm{Ph}-\mathrm{CH}), 135.6(\mathrm{C}, \mathrm{Ph}-\mathrm{C} 1), 142.6(\mathrm{C}, \mathrm{C} 4)$; HRMS (ESI), calcd for $\left(\mathrm{C}_{15} \mathrm{H}_{18} \mathrm{~N}_{4}+\mathrm{H}^{+}\right) 255.1604$, found 255.1607.

4.1.26. $N$-Methyl-N-[2-(1-phenethyl-1H-1,2,3-triazol-4-yl)ethyl]-N-propargylamine 34 It was prepared as described for 31. From amine 28 (384 mg, $1.67 \mathrm{mmol}), \mathrm{Cs}_{2} \mathrm{CO}_{3}(543$ $\mathrm{mg}, 1.67 \mathrm{mmol})$, and propargyl bromide $(80 \%$ solution in toluene, $0.25 \mathrm{~mL}, 1.68$ $\mathrm{mmol}$ ), and stirring at $\mathrm{rt}$ for $2 \mathrm{~h}$, propargylamine 34 (295 $\mathrm{mg}, 66 \%$ yield) was obtained as a yellow oil, without the need of chromatographic purification; $R_{f} 0.75\left(\mathrm{CH}_{2} \mathrm{Cl}_{2} /\right.$ $\mathrm{MeOH} / 50 \%$ aq. $\mathrm{NH}_{4} \mathrm{OH}$ 9.5:0.5:0.05).

34 $\cdot \mathrm{HCl}$ : brown solid, mp $157-159^{\circ} \mathrm{C}$; IR (ATR) v 3500-2300 (max at 3471, 3369, 3203, 3094, 3059, 3023, 2925, 2842, 2584, 2532, ${ }^{+} \mathrm{N}-\mathrm{H}, \equiv \mathrm{C}-\mathrm{H}$, and $\left.\mathrm{C}-\mathrm{H}\right), 2126(\mathrm{C} \equiv \mathrm{C})$ $\mathrm{cm}^{-1} ;{ }^{1} \mathrm{H}$ NMR (400 MHz, CD $\left.\mathrm{OD}\right) \delta 3.03\left(\mathrm{~s}, 3 \mathrm{H}, \mathrm{N}-\mathrm{CH}_{3}\right), 3.27(\mathrm{t}, J=7.2 \mathrm{~Hz}, 2 \mathrm{H}$, $1-\mathrm{CH}_{2}-\mathrm{CH}_{2}-\mathrm{Ph}$ ), 3.32 (t, $\left.J=7.2 \mathrm{~Hz}, 2 \mathrm{H}, 4-\mathrm{CH}_{2}-\mathrm{CH}_{2}-\mathrm{N}\right), 3.42$ (t, $J=2.4 \mathrm{~Hz}, 1 \mathrm{H}$, propargyl $\mathrm{CH}), 3.61\left(\mathrm{~m}, 2 \mathrm{H}, 4-\mathrm{CH}_{2}-\mathrm{CH}_{2}-\mathrm{N}\right), 4.23\left(\mathrm{~d}, J=2.4 \mathrm{~Hz}, 2 \mathrm{H}\right.$, propargyl $\left.\mathrm{CH}_{2}\right)$, 4.78 (t, $J=7.2 \mathrm{~Hz}, 2 \mathrm{H}, 1-\mathrm{CH}_{2}-\mathrm{CH}_{2}-\mathrm{Ph}$ ), 4.98 (s, ${ }^{+} \mathrm{NH}$ ), 7.16-7.30 (complex signal, $5 \mathrm{H}, \mathrm{Ph}-\mathrm{CH}), 8.22(\mathrm{~s}, 1 \mathrm{H}, 5-\mathrm{H}) ;{ }^{13} \mathrm{C} \mathrm{NMR}\left(100.6 \mathrm{MHz}, \mathrm{CD}_{3} \mathrm{OD}\right) \delta 20.8\left(\mathrm{CH}_{2}\right.$, $\left.4-\mathrm{CH}_{2}-\mathrm{CH}_{2}-\mathrm{N}\right), 36.8\left(\mathrm{CH}_{2}, 1-\mathrm{CH}_{2}-\mathrm{CH}_{2}-\mathrm{Ph}\right), 40.7\left(\mathrm{CH}_{3}, \mathrm{~N}-\mathrm{CH}_{3}\right), 46.4\left(\mathrm{CH}_{2}\right.$, propargyl $\left.\mathrm{CH}_{2}\right), 54.3\left(\mathrm{CH}_{2}, 1-\mathrm{CH}_{2}-\mathrm{CH}_{2}-\mathrm{Ph}\right), 54.6\left(\mathrm{CH}_{2}, 4-\mathrm{CH}_{2}-\mathrm{CH}_{2}-\mathrm{N}\right), 72.6(\mathrm{C}$, propargyl C), $81.8(\mathrm{CH}$, propargyl $\mathrm{CH}), 127.1(\mathrm{CH}, \mathrm{C} 5), 128.2(\mathrm{CH}), 129.8(4 \mathrm{CH})$ $(\mathrm{Ph}-\mathrm{CH}), 138.0(\mathrm{C}, \mathrm{Ph}-\mathrm{C1}), 141.5(\mathrm{C}, \mathrm{C} 4)$; HRMS (ESI), calcd for $\left(\mathrm{C}_{16} \mathrm{H}_{20} \mathrm{~N}_{4}+\mathrm{H}^{+}\right)$ 269.1761; found 269.1758.

\subsubsection{N-[(1-Benzyl-1H-1,2,3-triazol-4-yl)methyl]-N-methyl-N-propargylamine 35}

It was prepared as described for 31. From amine 29 (420 mg, $2.08 \mathrm{mmol}), \mathrm{Cs}_{2} \mathrm{CO}_{3}(676$ $\mathrm{mg}, 2.07 \mathrm{mmol})$, and propargyl bromide (80\% solution in toluene, $0.31 \mathrm{~mL}, 2.08$ $\mathrm{mmol})$, and stirring at $\mathrm{rt}$ for $3 \mathrm{~h}$, an oily residue $(451 \mathrm{mg}$ ) was obtained and subjected to purification through column chromatography (40-60 $\mu \mathrm{m}$ silica gel, $\mathrm{CH}_{2} \mathrm{Cl}_{2} / \mathrm{MeOH}$ mixtures, gradient elution). On elution with $\mathrm{CH}_{2} \mathrm{Cl}_{2} / \mathrm{MeOH}$ 98:2, propargylamine 35 (195 mg, 39\% yield) was isolated as a colorless oil; $R_{f} 0.48\left(\mathrm{CH}_{2} \mathrm{Cl}_{2} / \mathrm{MeOH}\right.$ 9.75:0.25). 35 $\mathrm{HCl}$ : pale brown solid, mp 121-123 ${ }^{\circ} \mathrm{C}$; IR (ATR) v 3500-2300 (max at 3193, 3126, 2976, 2961, 2692, 2578, ${ }^{+} \mathrm{N}-\mathrm{H}, \equiv \mathrm{C}-\mathrm{H}$, and $\left.\mathrm{C}-\mathrm{H}\right), 2118(\mathrm{C} \equiv \mathrm{C}) \mathrm{cm}^{-1} ;{ }^{1} \mathrm{H}$ NMR (400 $\left.\mathrm{MHz}, \mathrm{CD}_{3} \mathrm{OD}\right) \delta 2.96\left(\mathrm{~s}, 3 \mathrm{H}, \mathrm{N}-\mathrm{CH}_{3}\right), 3.40(\mathrm{t}, J=2.8 \mathrm{~Hz}, 1 \mathrm{H}$, propargyl $\mathrm{CH}), 4.15(\mathrm{~d}$, 
$J=2.8 \mathrm{~Hz}, 2 \mathrm{H}$, propargyl $\left.\mathrm{CH}_{2}\right), 4.57\left(\mathrm{~s}, 2 \mathrm{H}, 4-\mathrm{CH}_{2}-\mathrm{N}\right), 4.86\left(\mathrm{~s},{ }^{+} \mathrm{NH}\right), 5.66(\mathrm{~s}, 2 \mathrm{H}$, 1- $\mathrm{CH}_{2}-\mathrm{Ph}$ ), 7.32-7.41 (complex signal, 5H, $\left.\mathrm{Ph}-\mathrm{CH}\right), 8.27(\mathrm{~s}, 1 \mathrm{H}, 5-\mathrm{H}) ;{ }^{13} \mathrm{C} \mathrm{NMR}$ $\left(100.6 \mathrm{MHz}, \mathrm{CD}_{3} \mathrm{OD}\right) \delta 40.2\left(\mathrm{CH}_{3}, \mathrm{~N}-\mathrm{CH}_{3}\right), 45.7\left(\mathrm{CH}_{2}\right.$, propargyl $\left.\mathrm{CH}_{2}\right), 50.3\left(\mathrm{CH}_{2}\right.$, 4- $\left.\mathrm{CH}_{2}-\mathrm{N}\right), 55.1\left(\mathrm{CH}_{2}, 1-\mathrm{CH}_{2}-\mathrm{Ph}\right), 72.9(\mathrm{C}$, propargyl $\mathrm{C}), 81.6(\mathrm{CH}$, propargyl $\mathrm{CH})$, $128.1(\mathrm{CH}, \mathrm{C} 5), 129.3(2 \mathrm{CH}), 129.7(\mathrm{CH}), 130.1(2 \mathrm{CH})(\mathrm{Ph}-\mathrm{CH}), 136.5(\mathrm{C}, \mathrm{Ph}-\mathrm{C1})$, 137.9 (C, C4); HRMS (ESI), calcd for $\left(\mathrm{C}_{14} \mathrm{H}_{16} \mathrm{~N}_{4}+\mathrm{H}^{+}\right)$241.1448, found 241.1444.

\subsubsection{N-Methyl-N-[(1-phenethyl-1H-1,2,3-triazol-4-yl)methyl]-N-propargylamine 36}

It was prepared as described for 31. From amine 30 (258 mg, $1.19 \mathrm{mmol}), \mathrm{Cs}_{2} \mathrm{CO}_{3}(199$ $\mathrm{mg}, 0.61 \mathrm{mmol})$, and propargyl bromide (80\% solution in toluene, $0.17 \mathrm{~mL}, 1.14$ $\mathrm{mmol}$ ), and stirring at $\mathrm{rt}$ for $3 \mathrm{~h}$, an oily residue (204 mg) was obtained and subjected to purification through column chromatography $\left(40-60 \mu \mathrm{m}\right.$ silica gel, $\mathrm{CH}_{2} \mathrm{Cl}_{2} /$ EtOAc mixtures, gradient elution). On elution with $\mathrm{CH}_{2} \mathrm{Cl}_{2} /$ EtOAc 0:100, propargylamine 36 (63 mg, 22\% yield) was isolated as a colorless oil; $R_{f} 0.26$ (EtOAc).

36 $\mathrm{HCl}$ : beige sticky solid, IR (ATR) v 3500-2300 (max at 3400, 3271, 3209, 3023, 2945, 2501, ${ }^{+} \mathrm{N}-\mathrm{H}, \equiv \mathrm{C}-\mathrm{H}$, and $\left.\mathrm{C}-\mathrm{H}\right), 2124(\mathrm{C} \equiv \mathrm{C}) \mathrm{cm}^{-1} ;{ }^{1} \mathrm{H}$ NMR $\left(400 \mathrm{MHz}, \mathrm{CD}_{3} \mathrm{OD}\right)$ $\delta 2.88\left(\mathrm{~s}, 3 \mathrm{H}, \mathrm{N}-\mathrm{CH}_{3}\right), 3.25\left(\mathrm{t}, J=7.2 \mathrm{~Hz}, 2 \mathrm{H}, 1-\mathrm{CH}_{2}-\mathrm{CH}_{2}-\mathrm{Ph}\right), 3.42$ (t, $J=2.4 \mathrm{~Hz}$, $1 \mathrm{H}$, propargyl $\mathrm{CH}), 4.02\left(\right.$ br s, $2 \mathrm{H}$, propargyl $\left.\mathrm{CH}_{2}\right), 4.51\left(\mathrm{~s}, 2 \mathrm{H}, 4-\mathrm{CH}_{2}-\mathrm{N}\right), 4.73(\mathrm{t}, J=$ $\left.7.2 \mathrm{~Hz}, 2 \mathrm{H}, 1-\mathrm{CH}_{2}-\mathrm{CH}_{2}-\mathrm{Ph}\right), 4.90\left(\mathrm{~s},{ }^{+} \mathrm{NH}\right), 7.14[\mathrm{dm}, J=8.4 \mathrm{~Hz}, 2 \mathrm{H}, \mathrm{Ph}-\mathrm{C} 2(6)-H]$, 7.17-7.29 [complex signal, $3 \mathrm{H}, \mathrm{Ph}-\mathrm{C} 3(5)-H, \mathrm{Ph}-\mathrm{C} 4-H), 8.02$ (s, $1 \mathrm{H}, 5-\mathrm{H}) ;{ }^{13} \mathrm{C} \mathrm{NMR}$ (100.6 MHz, CD $\left.\mathrm{CD}_{3} \mathrm{OD}\right) \delta 37.3\left(\mathrm{CH}_{2}, 1-\mathrm{CH}_{2}-\mathrm{CH}_{2}-\mathrm{Ph}\right), 40.1\left(\mathrm{CH}_{3}, \mathrm{~N}-\mathrm{CH}_{3}\right), 45.5\left(\mathrm{CH}_{2}\right.$, propargyl $\left.\mathrm{CH}_{2}\right), 50.2\left(\mathrm{CH}_{2}, 4-\mathrm{CH}_{2}-\mathrm{N}\right), 52.8\left(\mathrm{CH}_{2}, 1-\mathrm{CH}_{2}-\mathrm{CH}_{2}-\mathrm{Ph}\right), 72.8(\mathrm{C}$, propargyl C), $81.7(\mathrm{CH}$, propargyl $\mathrm{CH}), 128.0(\mathrm{CH}), 128.4(\mathrm{CH})(\mathrm{C} 5, \mathrm{Ph}-\mathrm{C} 4), 129.7$ (2CH), 129.9 (2CH) [Ph-C2(6), Ph-C3(5)], 137.0 (C, Ph-C1), 138.6 (C, C4); HRMS (ESI), calcd for $\left(\mathrm{C}_{15} \mathrm{H}_{18} \mathrm{~N}_{4}+\mathrm{H}^{+}\right) 255.1604$, found 255.1601.

\subsubsection{9. $N$-(tert-Butoxycarbonyl)- $N$-[(1-ethyl-5-methyl-1H-1,2,3-triazol-4-yl)methyl]-N-} methylamine 37

A solution of $17(420 \mathrm{mg}, 1.75 \mathrm{mmol})$ in anhydrous THF $(30 \mathrm{~mL})$ was cooled to $-78{ }^{\circ} \mathrm{C}$ and then treated with $n$-BuLi $(2.5 \mathrm{M}$ solution in hexanes, $1.05 \mathrm{~mL}, 2.62 \mathrm{mmol})$ and iodomethane $(0.43 \mathrm{~mL}, 980 \mathrm{mg}, 6.91 \mathrm{mmol})$. The reaction mixture was stirred at $-78{ }^{\circ} \mathrm{C}$ for $1 \mathrm{~h}$, then warmed to $\mathrm{rt}$ and stirred an additional $2 \mathrm{~h}$. The resulting solution was diluted with $\mathrm{H}_{2} \mathrm{O}(20 \mathrm{~mL})$ and extracted with $\mathrm{CH}_{2} \mathrm{Cl}_{2}(2 \times 40 \mathrm{~mL})$. The combined 
organic extracts were dried over anhydrous $\mathrm{Na}_{2} \mathrm{SO}_{4}$ and evaporated under reduced pressure. The resulting residue was washed with pentane $(3 \times 10 \mathrm{~mL})$ and dried under vacuum, to give compound 37 (427 mg, $96 \%$ yield) as a colorless oil; $R_{f} 0.57\left(\mathrm{CH}_{2} \mathrm{Cl}_{2}\right.$ / MeOH 9:1); IR (ATR) $v 1686(\mathrm{C}=\mathrm{O}) \mathrm{cm}^{-1} ;{ }^{1} \mathrm{H}$ NMR (400 MHz, $\left.\mathrm{CDCl}_{3}\right) \delta 1.46[\mathrm{~s}, 9 \mathrm{H}$, $\left.\mathrm{C}\left(\mathrm{CH}_{3}\right)_{3}\right]$, superimposed in part $1.47\left(\mathrm{t}, J=7.6 \mathrm{~Hz}, 3 \mathrm{H}, 1-\mathrm{CH}_{2}-\mathrm{CH}_{3}\right), 2.31(\mathrm{~s}, 3 \mathrm{H}$, 5- $\left.\mathrm{CH}_{3}\right), 2.85\left(\mathrm{~s}, 3 \mathrm{H}, \mathrm{N}-\mathrm{CH}_{3}\right), 4.26\left(\mathrm{q}, J=7.6 \mathrm{~Hz}, 2 \mathrm{H}, 1-\mathrm{CH}_{2}-\mathrm{CH}_{3}\right), 4.48(\mathrm{~s}, 2 \mathrm{H}$,

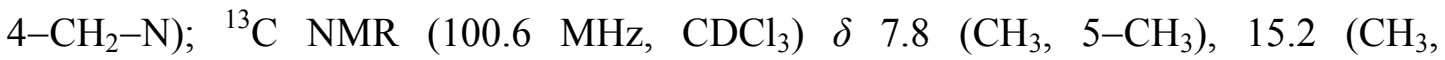
1- $\left.\mathrm{CH}_{2}-\mathrm{CH}_{3}\right), 28.5\left[3 \mathrm{CH}_{3}, \mathrm{C}\left(\mathrm{CH}_{3}\right)_{3}\right], 34.0\left(\mathrm{CH}_{3}, \mathrm{~N}-\mathrm{CH}_{3}\right), 42.7\left(\mathrm{CH}_{2}, 4-\mathrm{CH}_{2}-\mathrm{N}\right), 43.1$ $\left(\mathrm{CH}_{2}, 1-\mathrm{CH}_{2}-\mathrm{CH}_{3}\right), 79.8\left[\mathrm{C}, \mathrm{C}\left(\mathrm{CH}_{3}\right)_{3}\right], 130.5$ (C, C5), $142.0(\mathrm{C}, \mathrm{C} 4), 156.0(\mathrm{C}$, NCOO); HRMS (ESI), calcd for $\left(\mathrm{C}_{12} \mathrm{H}_{22} \mathrm{~N}_{4} \mathrm{O}_{2}+\mathrm{H}^{+}\right)$255.1816, found 255.1811.

4.1.30. $N$-(tert-Butoxycarbonyl)-N-[2-(1-ethyl-5-methyl-1H-1,2,3-triazol-4-yl)ethyl]-Nmethylamine $\mathbf{3 8}$

It was prepared as described for 37. From 18 (942 mg, $3.71 \mathrm{mmol}), n$-BuLi (2.5 M solution in hexanes, $2.97 \mathrm{~mL}, 7.42 \mathrm{mmol})$ and iodomethane $(0.92 \mathrm{~mL}, 2.10 \mathrm{~g}, 14.8$ mmol), compound 38 ( $895 \mathrm{mg}, 90 \%$ yield) was obtained as a colorless oil; $R_{f} 0.57$ $\left(\mathrm{CH}_{2} \mathrm{Cl}_{2} / \mathrm{MeOH} 9: 1\right)$; IR (ATR) $v 1685(\mathrm{C}=\mathrm{O}) \mathrm{cm}^{-1} ;{ }^{1} \mathrm{H}$ NMR $\left(400 \mathrm{MHz}, \mathrm{CDCl}_{3}\right) \delta$ $1.40\left[\mathrm{~s}, 9 \mathrm{H}, \mathrm{C}\left(\mathrm{CH}_{3}\right)_{3}\right], 1.44\left(\mathrm{t}, J=7.6 \mathrm{~Hz}, 3 \mathrm{H}, 1-\mathrm{CH}_{2}-\mathrm{CH}_{3}\right), 2.20\left(\mathrm{~s}, 3 \mathrm{H}, 5-\mathrm{CH}_{3}\right), 2.77$ (s, $\left.3 \mathrm{H}, \mathrm{N}-\mathrm{CH}_{3}\right), 2.82\left(\mathrm{~m}, 2 \mathrm{H}, 4-\mathrm{CH}_{2}-\mathrm{CH}_{2}-\mathrm{N}\right), 3.43\left(\mathrm{t}, J=7.2 \mathrm{~Hz}, 4-\mathrm{CH}_{2}-\mathrm{CH}_{2}-\mathrm{N}\right)$, $4.23\left(\mathrm{q}, J=7.6 \mathrm{~Hz}, 2 \mathrm{H}, 1-\mathrm{CH}_{2}-\mathrm{CH}_{3}\right) ;{ }^{13} \mathrm{C} \mathrm{NMR}\left(100.6 \mathrm{MHz}, \mathrm{CDCl}_{3}\right) \delta 7.7\left(\mathrm{CH}_{3}\right.$, 5- $\left.\mathrm{CH}_{3}\right), 15.3\left(\mathrm{CH}_{3}, 1-\mathrm{CH}_{2}-\mathrm{CH}_{3}\right), 23.7\left(\right.$ br $\left.\mathrm{CH}_{2}\right), 24.1\left(\right.$ br $\left.\mathrm{CH}_{2}\right)\left(4-\mathrm{CH}_{2}-\mathrm{CH}_{2}-\mathrm{N}\right), 28.5$ $\left[3 \mathrm{CH}_{3}, \mathrm{C}\left(\mathrm{CH}_{3}\right)_{3}\right], 34.7\left(\right.$ br $\left.\mathrm{CH}_{3}\right), 35.0\left(\right.$ br $\left.\mathrm{CH}_{3}\right)\left(\mathrm{N}-\mathrm{CH}_{3}\right), 43.0\left(\mathrm{CH}_{2}, 1-\mathrm{CH}_{2}-\mathrm{CH}_{3}\right), 48.7$ (br $\left.\left.\mathrm{CH}_{2}\right), 49.2(\text { br CH})_{2}\right)\left(4-\mathrm{CH}_{2}-\mathrm{CH}_{2}-\mathrm{N}\right), 79.4\left[\mathrm{C}, \mathrm{C}\left(\mathrm{CH}_{3}\right)_{3}\right], 129.2$ (br C), 129.4 (br C) (C5), 142.2 (C, C4), 155.7 (C, NCOO); HRMS (ESI), calcd for $\left(\mathrm{C}_{13} \mathrm{H}_{24} \mathrm{~N}_{4} \mathrm{O}_{2}+\mathrm{H}^{+}\right.$) 269.1972, found 269.1974.

4.1.31. $N$-(tert-Butoxycarbonyl)-N-methyl-N-\{2-[5-methyl-1-( $\alpha$-methyl)benzyl-1H-1,2,3triazol-4-yl]ethyl\}amine 39

It was prepared as described for 37. From 19 (356 mg, $1.13 \mathrm{mmol}), n$-BuLi (2.5 M solution in hexanes, $0.90 \mathrm{~mL}, 2.25 \mathrm{mmol})$ and iodomethane $(0.28 \mathrm{~mL}, 638 \mathrm{mg}, 4.50$ mmol), compound 39 (306 mg, 79\% yield) was obtained as a yellowish oil; IR (ATR) $v$ 1686, $1681(\mathrm{C}=\mathrm{O}) \mathrm{cm}^{-1} ;{ }^{1} \mathrm{H} \mathrm{NMR}\left(400 \mathrm{MHz}, \mathrm{CDCl}_{3}\right) \delta 1.41\left[\mathrm{~s}, 9 \mathrm{H}, \mathrm{C}\left(\mathrm{CH}_{3}\right)_{3}\right], 2.019[\mathrm{~d}$, $\left.J=7.2 \mathrm{~Hz}, 3 \mathrm{H}, 1-\mathrm{CH}\left(\mathrm{CH}_{3}\right)-\mathrm{Ph}\right], 2.023\left(\mathrm{~s}, 3 \mathrm{H}, 5-\mathrm{CH}_{3}\right), 2.76\left(\mathrm{~s}, 3 \mathrm{H}, \mathrm{N}-\mathrm{CH}_{3}\right), 2.81(\mathrm{~m}$, 
$\left.2 \mathrm{H}, 4-\mathrm{CH}_{2}-\mathrm{CH}_{2}-\mathrm{N}\right), 3.47$ (m, 2H, 4- $\left.\mathrm{CH}_{2}-\mathrm{CH}_{2}-\mathrm{N}\right), 5.49$ [q, $J=7.2 \mathrm{~Hz}, 1 \mathrm{H}$, 1- $\mathrm{CH}\left(\mathrm{CH}_{3}\right)-\mathrm{Ph}$ ], 7.14 [br d, $J=7.2 \mathrm{~Hz}, \mathrm{Ph}-\mathrm{C} 2(6)-H$ ], 7.24-7.34 [complex signal, 3H, $\mathrm{Ph}-\mathrm{C} 3(5)-H, \mathrm{Ph}-\mathrm{C} 4-H]$; HRMS (ESI), calcd for $\left(\mathrm{C}_{19} \mathrm{H}_{28} \mathrm{~N}_{4} \mathrm{O}_{2}+\mathrm{H}^{+}\right) 345.2285$, found 345.2285 .

4.1.32. N-(tert-Butoxycarbonyl)-N-[(5-butyl-1-methyl-1H-1,2,3-triazol-4-yl)methyl]-Nmethylamine 40

It was prepared as described for 37. From 21 (945 mg, $4.18 \mathrm{mmol}), n$-BuLi (2.5 M solution in hexanes, $3.36 \mathrm{~mL}, 8.40 \mathrm{mmol})$ and 1-bromobutane $(1.81 \mathrm{~mL}, 2.31 \mathrm{~g}, 16.9$ mmol), compound 40 (1.14 g, 97\% yield) was obtained as a yellowish oil; $R_{f} 0.87$ $\left(\mathrm{CH}_{2} \mathrm{Cl}_{2} / \mathrm{MeOH} / 50 \%\right.$ aq. $\mathrm{NH}_{4} \mathrm{OH}$ 9.5:0.5:0.05); IR (ATR) $v 1689(\mathrm{C}=\mathrm{O}) \mathrm{cm}^{-1} ;{ }^{1} \mathrm{H}$ $\operatorname{NMR}\left(400 \mathrm{MHz}, \mathrm{CDCl}_{3}\right) \delta 0.92\left(\mathrm{t}, J=7.2 \mathrm{~Hz}, 3 \mathrm{H}, 5-\mathrm{CH}_{2}-\mathrm{CH}_{2}-\mathrm{CH}_{2}-\mathrm{CH}_{3}\right), 1.35$ (tq, $J$ $\left.=J^{\prime}=7.2 \mathrm{~Hz}, \quad 2 \mathrm{H}, \quad 5-\mathrm{CH}_{2}-\mathrm{CH}_{2}-\mathrm{CH}_{2}-\mathrm{CH}_{3}\right)$, superimposed $1.40-1.52 \quad(2 \mathrm{H}$, 5- $\left.\mathrm{CH}_{2}-\mathrm{CH}_{2}-\mathrm{CH}_{2}-\mathrm{CH}_{3}\right), 1.44$ [s, 9H, $\left.\mathrm{C}\left(\mathrm{CH}_{3}\right)_{3}\right], 2.69$ (m, 2H, 5- $\mathrm{CH}_{2}-\mathrm{CH}_{2}-\mathrm{CH}_{2}-\mathrm{CH}_{3}$ ), 2.81 (s, 3H, N-CH $\left.\mathrm{CH}_{3}\right), 3.93$ (s, 3H, 1- $\left.\mathrm{CH}_{3}\right), 4.49$ (s, 2H, 4- $\left.\mathrm{CH}_{2}-\mathrm{N}\right)$; HRMS (ESI), calcd for $\left(\mathrm{C}_{14} \mathrm{H}_{26} \mathrm{~N}_{4} \mathrm{O}_{2}+\mathrm{H}^{+}\right)$283.2129; found 283.2123.

\subsubsection{3. $N$-(tert-Butoxycarbonyl)-N-methyl-N-[2-(5-ethyl-1-phenyl-1H-1,2,3-triazol-4- yl)ethyl]amine 41}

It was prepared as described for 37. From 22 (542 mg, $1.79 \mathrm{mmol}), n$-BuLi (2.5 M solution in hexanes, $1.43 \mathrm{~mL}, 3.57 \mathrm{mmol})$ and bromoethane $(0.53 \mathrm{~mL}, 774 \mathrm{mg}, 7.10$ mmol), compound 41 (578 mg, 98\% yield) was obtained as a yellowish oil; $R_{f} 0.86$ $\left(\mathrm{CH}_{2} \mathrm{Cl}_{2} / \mathrm{MeOH} / 50 \%\right.$ aq. $\mathrm{NH}_{4} \mathrm{OH}$ 9:1:0.05); IR (ATR) $v$ 1688, $1683(\mathrm{C}=\mathrm{O}) \mathrm{cm}^{-1} ;{ }^{1} \mathrm{H}$ NMR (400 MHz, $\left.\mathrm{CDCl}_{3}\right) \delta 1.05\left(\mathrm{t}, J=7.6 \mathrm{~Hz}, 3 \mathrm{H}, 5-\mathrm{CH}_{2}-\mathrm{CH}_{3}\right), 1.45[\mathrm{~s}, 9 \mathrm{H}$, $\left.\mathrm{C}\left(\mathrm{CH}_{3}\right)_{3}\right], 2.70\left(\mathrm{~m}, 2 \mathrm{H}, \quad 5-\mathrm{CH}_{2}-\mathrm{CH}_{3}\right), 2.88\left(\mathrm{~s}, 3 \mathrm{H}, \mathrm{N}-\mathrm{CH}_{3}\right), 2.93(\mathrm{~m}, 2 \mathrm{H}$, 4- $\left.\mathrm{CH}_{2}-\mathrm{CH}_{2}-\mathrm{N}\right), 3.58$ (t, $\left.J=7.6 \mathrm{~Hz}, 2 \mathrm{H}, 4-\mathrm{CH}_{2}-\mathrm{CH}_{2}-\mathrm{N}\right), 7.42\left[\mathrm{ddm}, J=8.0 \mathrm{~Hz}, J^{\prime}=\right.$ $2.4 \mathrm{~Hz}, 2 \mathrm{H}, \mathrm{Ph}-\mathrm{C} 2(6)-H], 7.48-7.57$ [complex signal, 3H, $\mathrm{Ph}-\mathrm{C} 3(5)-H, \mathrm{Ph}-\mathrm{C} 4-H$ ]; HRMS (ESI), calcd for $\left(\mathrm{C}_{18} \mathrm{H}_{26} \mathrm{~N}_{4} \mathrm{O}_{2}+\mathrm{H}^{+}\right)$331.2129; found 331.2128.

4.1.34. $N$-(tert-Butoxycarbonyl)-N-methyl-N-[2-(1-phenyl-5-propyl-1H-1,2,3-triazol-4yl)ethyl]amine $\mathbf{4 2}$

It was prepared as described for 37. From 22 (577 mg, $1.91 \mathrm{mmol}), n$-BuLi (2.5 M solution in hexanes, $1.53 \mathrm{~mL}, 3.82 \mathrm{mmol})$ and 1-bromopropane $(0.69 \mathrm{~mL}, 934 \mathrm{mg}, 7.60$ 
mmol), compound 42 (634 mg, 96\% yield) was obtained as an orange oil; $R_{f} 0.84$ $\left(\mathrm{CH}_{2} \mathrm{Cl}_{2} / \mathrm{MeOH} / 50 \%\right.$ aq. $\mathrm{NH}_{4} \mathrm{OH}$ 9:1:0.05); IR (ATR) $v 1692,1682(\mathrm{C}=\mathrm{O}) \mathrm{cm}^{-1} ;{ }^{1} \mathrm{H}$ $\operatorname{NMR}\left(400 \mathrm{MHz}, \mathrm{CDCl}_{3}\right) \delta 0.82\left(\mathrm{t}, J=7.6 \mathrm{~Hz}, 3 \mathrm{H}, 5-\mathrm{CH}_{2}-\mathrm{CH}_{2}-\mathrm{CH}_{3}\right)$, superimposed in part $1.42\left(\mathrm{tt}, J=J^{\prime}=7.6 \mathrm{~Hz}, 2 \mathrm{H}, 5-\mathrm{CH}_{2}-\mathrm{CH}_{2}-\mathrm{CH}_{3}\right), 1.46\left[\mathrm{~s}, 9 \mathrm{H}, \mathrm{C}\left(\mathrm{CH}_{3}\right)_{3}\right], 2.63(\mathrm{~m}$, $\left.2 \mathrm{H}, 5-\mathrm{CH}_{2}-\mathrm{CH}_{2}-\mathrm{CH}_{3}\right), 2.88\left(\mathrm{~s}, 3 \mathrm{H}, \mathrm{N}-\mathrm{CH}_{3}\right), 2.93\left(\mathrm{~m}, 2 \mathrm{H}, 4-\mathrm{CH}_{2}-\mathrm{CH}_{2}-\mathrm{N}\right), 3.59$ (t, $J$ $\left.=7.2 \mathrm{~Hz}, 2 \mathrm{H}, 4-\mathrm{CH}_{2}-\mathrm{CH}_{2}-\mathrm{N}\right), 7.41\left[\mathrm{ddm}, J=8.0 \mathrm{~Hz}, J^{\prime}=2.4 \mathrm{~Hz}, 2 \mathrm{H}, \mathrm{Ph}-\mathrm{C} 2(6)-H\right]$, 7.48-7.56 [complex signal, 3H, $\mathrm{Ph}-\mathrm{C} 3(5)-H, \mathrm{Ph}-\mathrm{C} 4-H$ ]; HRMS (ESI), calcd for $\left(\mathrm{C}_{19} \mathrm{H}_{28} \mathrm{~N}_{4} \mathrm{O}_{2}+\mathrm{H}^{+}\right)$345.2285, found 345.2286.

\subsubsection{N-(tert-Butoxycarbonyl)-N-methyl-N-[2-(5-butyl-1-phenyl-1H-1,2,3-triazol-4- yl)ethyl]amine 43}

It was prepared as described for 37. From 22 (590 mg, $1.95 \mathrm{mmol}), n$-BuLi (2.5 M solution in hexanes, $1.56 \mathrm{~mL}, 3.90 \mathrm{mmol})$ and 1-bromobutane $(0.84 \mathrm{~mL}, 1.07 \mathrm{~g}, 7.82$ mmol), an oily residue $(678 \mathrm{mg})$ was obtained. After column chromatography purification of the residue (40-60 $\mu \mathrm{m}$ silica gel, $\mathrm{CH}_{2} \mathrm{Cl}_{2} / 50 \%$ aq. $\mathrm{NH}_{4} \mathrm{OH}$ 100:0.2), compound 43 (300 mg, 43\% yield) was isolated as a yellow oil; $R_{f} 0.82\left(\mathrm{CH}_{2} \mathrm{Cl}_{2} /\right.$ $\mathrm{MeOH} / 50 \%$ aq. $\mathrm{NH}_{4} \mathrm{OH}$ 9:1:0.05); IR (ATR) $v 1692(\mathrm{C}=\mathrm{O}) \mathrm{cm}^{-1} ;{ }^{1} \mathrm{H}$ NMR (400 MHz, $\left.\mathrm{CDCl}_{3}\right) \delta 0.79\left(\mathrm{t}, J=7.2 \mathrm{~Hz}, 3 \mathrm{H}, 5-\mathrm{CH}_{2}-\mathrm{CH}_{2}-\mathrm{CH}_{2}-\mathrm{CH}_{3}\right), 1.21$ (tq, $J=J^{\prime}=7.2 \mathrm{~Hz}$, $\left.2 \mathrm{H}, 5-\mathrm{CH}_{2}-\mathrm{CH}_{2}-\mathrm{CH}_{2}-\mathrm{CH}_{3}\right), 1.35\left(\mathrm{~m}, 2 \mathrm{H}, 5-\mathrm{CH}_{2}-\mathrm{CH}_{2}-\mathrm{CH}_{2}-\mathrm{CH}_{3}\right), 1.45[\mathrm{~s}, 9 \mathrm{H}$, $\left.\mathrm{C}\left(\mathrm{CH}_{3}\right)_{3}\right], 2.65\left(\mathrm{~m}, 2 \mathrm{H}, 5-\mathrm{CH}_{2}-\mathrm{CH}_{2}-\mathrm{CH}_{2}-\mathrm{CH}_{3}\right), 2.87\left(\mathrm{~s}, 3 \mathrm{H}, \mathrm{N}-\mathrm{CH}_{3}\right), 2.91(\mathrm{~m}, 2 \mathrm{H}$, 4- $\mathrm{CH}_{2}-\mathrm{CH}_{2}-\mathrm{N}$ ), 3.59 (t, $\left.J=7.6 \mathrm{~Hz}, 2 \mathrm{H}, 4-\mathrm{CH}_{2}-\mathrm{CH}_{2}-\mathrm{N}\right), 7.41\left[\mathrm{ddm}, J=8.0 \mathrm{~Hz}, J^{\prime}=\right.$ $2.4 \mathrm{~Hz}, 2 \mathrm{H}, \mathrm{Ph}-\mathrm{C} 2(6)-H$ ], 7.47-7.56 [complex signal, 3H, Ph-C3(5)-H, Ph-C4-H]; HRMS (ESI), calcd for $\left(\mathrm{C}_{20} \mathrm{H}_{30} \mathrm{~N}_{4} \mathrm{O}_{2}+\mathrm{H}^{+}\right)$359.2442, found 359.2438.

\subsubsection{N-[(1-Ethyl-5-methyl-1H-1,2,3-triazol-4-yl)methyl]-N-methylamine 44}

It was prepared as described for 25 . From $37(370 \mathrm{mg}, 1.46 \mathrm{mmol})$ and $\mathrm{H}_{3} \mathrm{PO}_{4}(85 \%$ purity, $2.52 \mathrm{~mL}, 21.9 \mathrm{mmol}$ ), and stirring at $\mathrm{rt}$ for $4 \mathrm{~h}$, amine 44 (205 $\mathrm{mg}, 91 \%$ yield) was obtained as a colorless oil; $R_{f} 0.11\left(\mathrm{CH}_{2} \mathrm{Cl}_{2} / \mathrm{MeOH} / 50 \%\right.$ aq. $\mathrm{NH}_{4} \mathrm{OH}$ 9:1:0.05). 44. $\mathrm{HCl}$ : beige sticky solid, IR (ATR) v 3500-2400 (max at 3390, 2925, 2868, 2837, 2757, 2697, 2546, 2440, ${ }^{+} \mathrm{N}-\mathrm{H}$ and C-H) $\mathrm{cm}^{-1}$; ${ }^{1} \mathrm{H}$ NMR (400 MHz, CD $\left.3 \mathrm{OD}\right) \delta 1.48(\mathrm{t}$, $\left.J=7.2 \mathrm{~Hz}, 3 \mathrm{H}, 1-\mathrm{CH}_{2}-\mathrm{CH}_{3}\right), 2.41\left(\mathrm{~s}, 3 \mathrm{H}, 5-\mathrm{CH}_{3}\right), 2.76\left(\mathrm{~s}, 3 \mathrm{H}, \mathrm{N}-\mathrm{CH}_{3}\right), 4.28(\mathrm{~s}, 2 \mathrm{H}$, 4- $\left.\mathrm{CH}_{2}-\mathrm{N}\right), 4.38\left(\mathrm{q}, J=7.2 \mathrm{~Hz}, 2 \mathrm{H}, 1-\mathrm{CH}_{2}-\mathrm{CH}_{3}\right), 4.87\left(\mathrm{~s},{ }^{+} \mathrm{NH}_{2}\right) ;{ }^{13} \mathrm{C}$ NMR $(100.6$ $\left.\mathrm{MHz}, \mathrm{CD}_{3} \mathrm{OD}\right) \delta 7.7\left(\mathrm{CH}_{3}, 5-\mathrm{CH}_{3}\right), 15.2\left(\mathrm{CH}_{3}, 1-\mathrm{CH}_{2}-\mathrm{CH}_{3}\right), 33.1\left(\mathrm{CH}_{3}, \mathrm{~N}-\mathrm{CH}_{3}\right), 43.6$ 
$\left(\mathrm{CH}_{2}\right), 44.4\left(\mathrm{CH}_{2}\right)\left(1-\mathrm{CH}_{2}-\mathrm{CH}_{3}, 4-\mathrm{CH}_{2}-\mathrm{N}\right), 134.7$ (C, C5), 136.8 (C, C4); HRMS (ESI), calcd for $\left(\mathrm{C}_{7} \mathrm{H}_{14} \mathrm{~N}_{4}+\mathrm{H}^{+}\right)$155.1291, found 155.1288.

\subsubsection{7. $\mathrm{N}$-[2-(1-Ethyl-5-methyl-1H-1,2,3-triazol-4-yl)ethyl]-N-methylamine 45}

It was prepared as described for 25. From 38 (890 mg, $3.32 \mathrm{mmol})$ and $\mathrm{H}_{3} \mathrm{PO}_{4}(85 \%$ purity, $5.70 \mathrm{~mL}, 49.4 \mathrm{mmol}$ ), and stirring at rt for $1.5 \mathrm{~h}$, amine 45 (495 mg, 89\% yield) was obtained as a colorless oil; $R_{f} 0.10\left(\mathrm{CH}_{2} \mathrm{Cl}_{2} / \mathrm{MeOH} / 50 \%\right.$ aq. $\mathrm{NH}_{4} \mathrm{OH}$ 9:1:0.05). 45 $\mathrm{HCl}$ : beige sticky solid, IR (ATR) v 3500-2300 (max at 3374, 2981, 2937, 2746, 2692, 2449, 2359, ${ }^{+} \mathrm{N}-\mathrm{H}$ and $\mathrm{C}-\mathrm{H}$ st) $\mathrm{cm}^{-1}$; ${ }^{1} \mathrm{H}$ NMR (400 MHz, CD $\left.3 \mathrm{OD}\right) \delta 1.49$ (t, $J=$ $\left.7.2 \mathrm{~Hz}, 3 \mathrm{H}, 1-\mathrm{CH}_{2}-\mathrm{CH}_{3}\right), 2.38\left(\mathrm{~s}, 3 \mathrm{H}, 5-\mathrm{CH}_{3}\right), 2.76\left(\mathrm{~s}, 3 \mathrm{H}, \mathrm{N}-\mathrm{CH}_{3}\right), 3.09(\mathrm{t}, J=7.2$ $\left.\mathrm{Hz}, 2 \mathrm{H}, 4-\mathrm{CH}_{2}-\mathrm{CH}_{2}-\mathrm{N}\right), 3.34\left(\mathrm{t}, J=7.2 \mathrm{~Hz}, 2 \mathrm{H}, 4-\mathrm{CH}_{2}-\mathrm{CH}_{2}-\mathrm{N}\right), 4.40$ (q, $J=7.2 \mathrm{~Hz}$, $\left.2 \mathrm{H}, 1-\mathrm{CH}_{2}-\mathrm{CH}_{3}\right), 4.86\left(\mathrm{~s},{ }^{+} \mathrm{NH}_{2}\right) ;{ }^{13} \mathrm{C} \mathrm{NMR}\left(100.6 \mathrm{MHz}, \mathrm{CD}_{3} \mathrm{OD}\right) \delta 7.6\left(\mathrm{CH}_{3}, 5-\mathrm{CH}_{3}\right)$, $15.0\left(\mathrm{CH}_{3}, 1-\mathrm{CH}_{2}-\mathrm{CH}_{3}\right), 22.2\left(\mathrm{CH}_{2}, 4-\mathrm{CH}_{2}-\mathrm{CH}_{2}-\mathrm{N}\right), 33.7\left(\mathrm{CH}_{3}, \mathrm{~N}-\mathrm{CH}_{3}\right), 44.9\left(\mathrm{CH}_{2}\right.$, 1- $\left.\mathrm{CH}_{2}-\mathrm{CH}_{3}\right), 49.0\left(4-\mathrm{CH}_{2}-\mathrm{CH}_{2}-\mathrm{N}\right), 133.4$ (C, C5), 140.1 (C, C4); HRMS (ESI), calcd for $\left(\mathrm{C}_{8} \mathrm{H}_{16} \mathrm{~N}_{4}+\mathrm{H}^{+}\right)$169.1448, found 169.1452 .

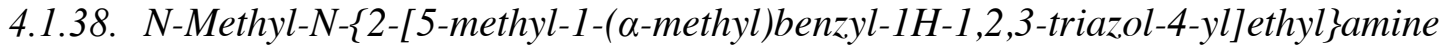
46

It was prepared as described for 25 . From $39(306 \mathrm{mg}, 0.89 \mathrm{mmol})$ and $\mathrm{H}_{3} \mathrm{PO}_{4}(85 \%$ purity, $1.60 \mathrm{~mL}, 13.9 \mathrm{mmol})$, amine 46 (215 $\mathrm{mg}$, quantitative yield) was obtained as a yellow oil; $R_{f} 0.33\left(\mathrm{CH}_{2} \mathrm{Cl}_{2} / \mathrm{MeOH} / 50 \%\right.$ aq. $\mathrm{NH}_{4} \mathrm{OH}$ 9:1:0.05).

46. HCl: yellow sticky solid, IR (ATR) v 3400-2400 (max at 3373, 2950, 2709, 2434, ${ }^{+} \mathrm{N}-\mathrm{H}$ and $\left.\mathrm{C}-\mathrm{H}\right) \mathrm{cm}^{-1} ;{ }^{1} \mathrm{H}$ NMR $\left(400 \mathrm{MHz}, \mathrm{CD}_{3} \mathrm{OD}\right) \delta 2.01[\mathrm{~d}, J=7.2 \mathrm{~Hz}, 3 \mathrm{H}$, $\left.1-\mathrm{CH}\left(\mathrm{CH}_{3}\right)-\mathrm{Ph}\right], 2.30\left(\mathrm{~s}, 3 \mathrm{H}, 5-\mathrm{CH}_{3}\right), 2.76\left(\mathrm{~s}, 3 \mathrm{H}, \mathrm{N}-\mathrm{CH}_{3}\right), 3.19(\mathrm{t}, J=7.2 \mathrm{~Hz}, 2 \mathrm{H}$, 4- $\left.\mathrm{CH}_{2}-\mathrm{CH}_{2}-\mathrm{N}\right), 3.36\left(\mathrm{t}, J=7.2 \mathrm{~Hz}, 2 \mathrm{H}, 4-\mathrm{CH}_{2}-\mathrm{CH}_{2}-\mathrm{N}\right), 4.94\left(\mathrm{~s},{ }^{+} \mathrm{NH}_{2}\right), 5.93(\mathrm{q}, J=$ $\left.7.2 \mathrm{~Hz}, 1 \mathrm{H}, 1-\mathrm{CH}\left(\mathrm{CH}_{3}\right)-\mathrm{Ph}\right], 7.30[\mathrm{dm}, J=8.4 \mathrm{~Hz}, 2 \mathrm{H}, \mathrm{Ph}-\mathrm{C} 2(6)-H], 7.36-7.42$ [complex signal, 3H, Ph-C3(5)-H, Ph-C4-H]; ${ }^{13} \mathrm{C}$ NMR (100.6 MHz, $\left.\mathrm{CD}_{3} \mathrm{OD}\right) \delta 8.2$ $\left(\mathrm{CH}_{3}, 5-\mathrm{CH}_{3}\right), 21.7\left[\mathrm{CH}_{3}, 1-\mathrm{CH}\left(\mathrm{CH}_{3}\right)-\mathrm{Ph}\right], 21.9\left(\mathrm{CH}_{2}, 4-\mathrm{CH}_{2}-\mathrm{CH}_{2}-\mathrm{N}\right), 33.7\left(\mathrm{CH}_{3}\right.$, $\left.\mathrm{N}-\mathrm{CH}_{3}\right), 53.8\left(\mathrm{CH}_{2}, 4-\mathrm{CH}_{2}-\mathrm{CH}_{2}-\mathrm{N}\right), 61.5\left[\mathrm{CH}, 1-\mathrm{CH}\left(\mathrm{CH}_{3}\right)-\mathrm{Ph}\right], 127.5(2 \mathrm{CH}), 129.7$ (CH), 130.2 (2CH) (Ph-CH), 135.6 (C, Ph-C1), 139.3 (C, C5), 140.5 (C, C4); HRMS (ESI), calcd for $\left(\mathrm{C}_{14} \mathrm{H}_{20} \mathrm{~N}_{4}+\mathrm{H}^{+}\right)$245.1761, found 245.1762. 
It was prepared as described for 25. From 40 (954 mg, $3.38 \mathrm{mmol})$ and $\mathrm{H}_{3} \mathrm{PO}_{4}(85 \%$ purity, $5.84 \mathrm{~mL}, 50.7 \mathrm{mmol})$, amine $47(620 \mathrm{mg}$, quantitative yield) was obtained as a yellow oil; $R_{f} 0.06\left(\mathrm{CH}_{2} \mathrm{Cl}_{2} / \mathrm{MeOH} / 50 \%\right.$ aq. $\mathrm{NH}_{4} \mathrm{OH}$ 9.5:0.5:0.05).

47. $\mathrm{HCl}$ : beige sticky solid, IR (ATR) v 3400-2500 (max at 3372, 2957, 2930, 2863, 2711, ${ }^{+} \mathrm{N}-\mathrm{H}$ and $\left.\mathrm{C}-\mathrm{H}\right) \mathrm{cm}^{-1}$; ${ }^{1} \mathrm{H}$ NMR $\left(400 \mathrm{MHz}, \mathrm{CD}_{3} \mathrm{OD}\right) \delta 0.97(\mathrm{t}, J=7.2 \mathrm{~Hz}, 3 \mathrm{H}$, 5- $\left.\mathrm{CH}_{2}-\mathrm{CH}_{2}-\mathrm{CH}_{2}-\mathrm{CH}_{3}\right), 1.42\left(\mathrm{tq}, J=J^{\prime}=7.2 \mathrm{~Hz}, 2 \mathrm{H}, 5-\mathrm{CH}_{2}-\mathrm{CH}_{2}-\mathrm{CH}_{2}-\mathrm{CH}_{3}\right), 1.58$ (tt, $\left.J=7.6 \mathrm{~Hz}, J^{\prime}=7.2 \mathrm{~Hz}, 2 \mathrm{H}, 5-\mathrm{CH}_{2}-\mathrm{CH}_{2}-\mathrm{CH}_{2}-\mathrm{CH}_{3}\right), 2.79\left(\mathrm{~s}, 3 \mathrm{H}, \mathrm{N}-\mathrm{CH}_{3}\right), 2.87$ (t, $\left.J=7.6 \mathrm{~Hz}, 2 \mathrm{H}, 5-\mathrm{CH}_{2}-\mathrm{CH}_{2}-\mathrm{CH}_{2}-\mathrm{CH}_{3}\right), 4.05\left(\mathrm{~s}, 3 \mathrm{H}, 1-\mathrm{CH}_{3}\right), 4.31\left(\mathrm{~s}, 2 \mathrm{H}, 4-\mathrm{CH}_{2}-\mathrm{N}\right)$, $5.06\left(\mathrm{~s},{ }^{+} \mathrm{NH}_{2}\right) ;{ }^{13} \mathrm{C} \mathrm{NMR}\left(100.6 \mathrm{MHz}, \mathrm{CD}_{3} \mathrm{OD}\right) \delta 14.0\left(\mathrm{CH}_{3}, 5-\mathrm{CH}_{2}-\mathrm{CH}_{2}-\mathrm{CH}_{2}-\mathrm{CH}_{3}\right)$, $22.9\left(\mathrm{CH}_{2}\right), 23.4\left(\mathrm{CH}_{2}\right)\left(5-\mathrm{CH}_{2}-\mathrm{CH}_{2}-\mathrm{CH}_{2}-\mathrm{CH}_{3}\right), 31.6\left(\mathrm{CH}_{2}, 5-\mathrm{CH}_{2}-\mathrm{CH}_{2}-\mathrm{CH}_{2}-\mathrm{CH}_{3}\right)$, $33.3\left(\mathrm{CH}_{3}, \mathrm{~N}-\mathrm{CH}_{3}\right), 35.5\left(\mathrm{CH}_{3}, 1-\mathrm{CH}_{3}\right), 43.5\left(\mathrm{CH}_{2}, 4-\mathrm{CH}_{2}-\mathrm{N}\right), 136.5(\mathrm{C}, \mathrm{C} 5), 139.3$ (C, C4); HRMS (ESI), calcd for $\left(\mathrm{C}_{9} \mathrm{H}_{18} \mathrm{~N}_{4}+\mathrm{H}^{+}\right)$183.1604, found 183.1599 .

\subsubsection{N-Methyl-N-[2-(5-ethyl-1-phenyl-1H-1,2,3-triazol-4-yl)ethyl]amine $\mathbf{4 8}$}

It was prepared as described for 25. From $41(517 \mathrm{mg}, 1.57 \mathrm{mmol})$ and $\mathrm{H}_{3} \mathrm{PO}_{4}(85 \%$ purity, $2.68 \mathrm{~mL}, 23.2 \mathrm{mmol}$ ), and stirring at $\mathrm{rt}$ for $3 \mathrm{~h}$, an orange oil (435 mg) was obtained and purified through column chromatography (40-60 $\mu \mathrm{m}$ silica gel, $\mathrm{CH}_{2} \mathrm{Cl}_{2}$ / $\mathrm{MeOH} / 50 \%$ aq. $\mathrm{NH}_{4} \mathrm{OH}$ mixtures, gradient elution). On elution with $\mathrm{CH}_{2} \mathrm{Cl}_{2} / \mathrm{MeOH} /$ 50\% aq. $\mathrm{NH}_{4} \mathrm{OH}$ 98:2:0.2 to 96:4:0.2, amine 48 (322 mg, 89\% yield) was obtained as a yellowish oil; $R_{f} 0.51\left(\mathrm{CH}_{2} \mathrm{Cl}_{2} / \mathrm{MeOH} / 50 \%\right.$ aq. $\mathrm{NH}_{4} \mathrm{OH}$ 9:1:0.05).

48. $\mathrm{HCl}$ : yellow sticky solid, IR (ATR) v 3400-2300 (max at 3380, 2964, 2930, 2873, 2741, 2434, 2351, ${ }^{+} \mathrm{N}-\mathrm{H}$ and $\left.\mathrm{C}-\mathrm{H}\right) \mathrm{cm}^{-1} ;{ }^{1} \mathrm{H}$ NMR (400 MHz, CD $\left.3 \mathrm{OD}\right) \delta 1.05(\mathrm{t}, J=$ $\left.7.6 \mathrm{~Hz}, 3 \mathrm{H}, 5-\mathrm{CH}_{2}-\mathrm{CH}_{3}\right)$, superimposed in part $2.80\left(\mathrm{q}, \mathrm{J}=7.6 \mathrm{~Hz}, 2 \mathrm{H}, 5-\mathrm{CH}_{2}-\mathrm{CH}_{3}\right)$, $2.81\left(\mathrm{~s}, 3 \mathrm{H}, \mathrm{N}-\mathrm{CH}_{3}\right), 3.20\left(\mathrm{t}, J=7.2 \mathrm{~Hz}, 2 \mathrm{H}, 4-\mathrm{CH}_{2}-\mathrm{CH}_{2}-\mathrm{N}\right), 3.45$ (t, $J=7.2 \mathrm{~Hz}, 2 \mathrm{H}$, 4- $\left.\mathrm{CH}_{2}-\mathrm{CH}_{2}-\mathrm{N}\right), 4.89$ (s, $\left.{ }^{+} \mathrm{NH}_{2}\right), 7.53$ [ddm, $J=7.6 \mathrm{~Hz}, J$ ' = 2.0 Hz, 2H, Ph-C2(6)-H], 7.62-7.67 [complex signal, $3 \mathrm{H}, \mathrm{Ph}-\mathrm{C} 3(5)-H, \mathrm{Ph}-\mathrm{C} 4-H$ ]; ${ }^{13} \mathrm{C}$ NMR (100.6 MHz, $\left.\mathrm{CD}_{3} \mathrm{OD}\right) \quad \delta \quad 13.4\left(\mathrm{CH}_{3}, \quad 5-\mathrm{CH}_{2}-\mathrm{CH}_{3}\right), \quad 16.9 \quad\left(\mathrm{CH}_{2}, \quad 5-\mathrm{CH}_{2}-\mathrm{CH}_{3}\right), 22.5 \quad\left(\mathrm{CH}_{2}\right.$, $\left.4-\mathrm{CH}_{2}-\mathrm{CH}_{2}-\mathrm{N}\right), 33.9\left(\mathrm{CH}_{3}, \mathrm{~N}-\mathrm{CH}_{3}\right), 49.3\left(\mathrm{CH}_{2}, 4-\mathrm{CH}_{2}-\mathrm{CH}_{2}-\mathrm{N}\right), 126.8(2 \mathrm{CH}), 130.9$ (2CH), $131.4(\mathrm{CH})(\mathrm{Ph}-\mathrm{CH}), 137.5$ (C, Ph-C1), 139.1 (C, C5), 140.5 (C, C4); HRMS (ESI), calcd for $\left(\mathrm{C}_{13} \mathrm{H}_{18} \mathrm{~N}_{4}+\mathrm{H}^{+}\right)$231.1604; found 231.1607.

\subsubsection{N-Methyl-N-[2-(1-phenyl-5-propyl-1H-1,2,3-triazol-4-yl)ethyl]amine 49}


It was prepared as described for 25. From $42(573 \mathrm{mg}, 1.66 \mathrm{mmol})$ and $\mathrm{H}_{3} \mathrm{PO}_{4}(85 \%$ purity, $2.85 \mathrm{~mL}, 24.7 \mathrm{mmol}$ ), and stirring at $\mathrm{rt}$ for $3 \mathrm{~h}$, an orange oil (564 mg) was obtained and purified through column chromatography (40-60 $\mu \mathrm{m}$ silica gel, $\mathrm{CH}_{2} \mathrm{Cl}_{2}$ / $\mathrm{MeOH} / 50 \%$ aq. $\mathrm{NH}_{4} \mathrm{OH}$ mixtures, gradient elution). On elution with $\mathrm{CH}_{2} \mathrm{Cl}_{2} / \mathrm{MeOH} /$ 50\% aq. $\mathrm{NH}_{4} \mathrm{OH}$ 99:1:0.2 to 95:5:0.2, amine 49 (270 mg, 67\% yield) was obtained as a yellowish oil; $R_{f} 0.52\left(\mathrm{CH}_{2} \mathrm{Cl}_{2} / \mathrm{MeOH} / 50 \%\right.$ aq. $\mathrm{NH}_{4} \mathrm{OH}$ 9:1:0.05).

49. $\mathrm{HCl}$ : yellow sticky solid, IR (ATR) v 3400-2400 (max at 3350, 2960, 2935, 2863, 2721, 2430, ${ }^{+} \mathrm{N}-\mathrm{H}$ and $\left.\mathrm{C}-\mathrm{H}\right) \mathrm{cm}^{-1} ;{ }^{1} \mathrm{H}$ NMR $\left(400 \mathrm{MHz}, \mathrm{CD}_{3} \mathrm{OD}\right) \delta 0.81(\mathrm{t}, J=7.6 \mathrm{~Hz}$, $\left.3 \mathrm{H}, 5-\mathrm{CH}_{2}-\mathrm{CH}_{2}-\mathrm{CH}_{3}\right), 1.42$ (tq, $\left.J=J^{\prime}=7.6 \mathrm{~Hz}, 2 \mathrm{H} 5-\mathrm{CH}_{2}-\mathrm{CH}_{2}-\mathrm{CH}_{3}\right), 2.74$ (t, $J=$ $\left.7.6 \mathrm{~Hz}, 2 \mathrm{H}, 5-\mathrm{CH}_{2}-\mathrm{CH}_{2}-\mathrm{CH}_{3}\right), 2.80\left(\mathrm{~s}, 3 \mathrm{H}, \mathrm{N}-\mathrm{CH}_{3}\right), 3.16(\mathrm{t}, J=7.2 \mathrm{~Hz}, 2 \mathrm{H}$, 4- $\left.\mathrm{CH}_{2}-\mathrm{CH}_{2}-\mathrm{N}\right), 3.43$ (t, $\left.J=7.2 \mathrm{~Hz}, 2 \mathrm{H}, 4-\mathrm{CH}_{2}-\mathrm{CH}_{2}-\mathrm{N}\right), 4.85\left(\mathrm{~s},{ }^{+} \mathrm{NH}_{2}\right), 7.48-7.65$ (complex signal, 5H, Ph- $\mathrm{CH}) ;{ }^{13} \mathrm{C}$ NMR (100.6 MHz, CD $\left.{ }_{3} \mathrm{OD}\right) \delta 13.9\left(\mathrm{CH}_{3}\right.$, 5- $\left.\mathrm{CH}_{2}-\mathrm{CH}_{2}-\mathrm{CH}_{3}\right), 22.6\left(\mathrm{CH}_{2}, 4-\mathrm{CH}_{2}-\mathrm{CH}_{2}-\mathrm{N}\right), 22.9\left(\mathrm{CH}_{2}, 5-\mathrm{CH}_{2}-\mathrm{CH}_{2}-\mathrm{CH}_{3}\right), 25.2$ $\left(\mathrm{CH}_{2}, 5-\mathrm{CH}_{2}-\mathrm{CH}_{2}-\mathrm{CH}_{3}\right), 33.8\left(\mathrm{CH}_{3}, \mathrm{~N}-\mathrm{CH}_{3}\right), 49.4\left(\mathrm{CH}_{2}, 4-\mathrm{CH}_{2}-\mathrm{CH}_{2}-\mathrm{N}\right) 126.8$ (2CH), 130.9 (2CH), $131.3(\mathrm{CH})(\mathrm{Ph}-\mathrm{CH}), 137.4$ (C, Ph-C1), 137.7 (C, C5), 141.3 (C, C4); HRMS (ESI), calcd for $\left(\mathrm{C}_{14} \mathrm{H}_{20} \mathrm{~N}_{4}+\mathrm{H}^{+}\right)$245.1761, found 245.1761.

\subsubsection{N-Methyl-N-[2-(5-butyl-1-phenyl-1H-1,2,3-triazol-4-yl)ethyl]amine 50}

It was prepared as described for 25 . From $43(265 \mathrm{mg}, 0.74 \mathrm{mmol})$ and $\mathrm{H}_{3} \mathrm{PO}_{4}(85 \%$ purity, $1.27 \mathrm{~mL}, 11.0 \mathrm{mmol}$ ), and stirring at $\mathrm{rt}$ for $3 \mathrm{~h}$, amine $\mathbf{5 0}$ (175 mg, 92\% yield) was obtained as a yellowish oil; $R_{f} 0.54\left(\mathrm{CH}_{2} \mathrm{Cl}_{2} / \mathrm{MeOH} / 50 \%\right.$ aq. $\mathrm{NH}_{4} \mathrm{OH}$ 9:1:0.05).

50. $\mathrm{HCl}$ : yellow oil, IR (ATR) $v$ 3400-2400 ( $\max$ at 3389, 2954, 2919, 2868, 2755, 2444, ${ }^{+} \mathrm{N}-\mathrm{H}$ and $\left.\mathrm{C}-\mathrm{H}\right) \mathrm{cm}^{-1}$; ${ }^{1} \mathrm{H}$ NMR $\left(400 \mathrm{MHz}, \mathrm{CD}_{3} \mathrm{OD}\right) \delta 0.80(\mathrm{t}, J=7.6 \mathrm{~Hz}, 3 \mathrm{H}$, $\left.5-\mathrm{CH}_{2}-\mathrm{CH}_{2}-\mathrm{CH}_{2}-\mathrm{CH}_{3}\right), 1.22\left(\mathrm{tq}, J=J^{\prime}=7.6 \mathrm{~Hz}, 2 \mathrm{H}, 5-\mathrm{CH}_{2}-\mathrm{CH}_{2}-\mathrm{CH}_{2}-\mathrm{CH}_{3}\right), 1.38$ $\left(\mathrm{tt}, J=J^{\prime}=7.6 \mathrm{~Hz}, 2 \mathrm{H}, 5-\mathrm{CH}_{2}-\mathrm{CH}_{2}-\mathrm{CH}_{2}-\mathrm{CH}_{3}\right), 2.77(\mathrm{t}, J=7.6 \mathrm{~Hz}, 2 \mathrm{H}$, $\left.5-\mathrm{CH}_{2}-\mathrm{CH}_{2}-\mathrm{CH}_{2}-\mathrm{CH}_{3}\right), 2.81\left(\mathrm{~s}, 3 \mathrm{H}, \mathrm{N}-\mathrm{CH}_{3}\right), 3.15(\mathrm{t}, J=7.2 \mathrm{~Hz}, 2 \mathrm{H}$, 4- $\left.\mathrm{CH}_{2}-\mathrm{CH}_{2}-\mathrm{N}\right), 3.44\left(\mathrm{t}, J=7.2 \mathrm{~Hz}, 2 \mathrm{H}, 4-\mathrm{CH}_{2}-\mathrm{CH}_{2}-\mathrm{N}\right), 4.85\left(\mathrm{~s},{ }^{+} \mathrm{NH}_{2}\right), 7.51[\mathrm{dm}, J=$ $8.0 \mathrm{~Hz}, 2 \mathrm{H}, \mathrm{Ph}-\mathrm{C} 2(6)-H]$, 7.61-7.66 [complex signal, 3H, Ph-C3(5)-H, Ph-C4-H]; ${ }^{13} \mathrm{C}$ NMR (100.6 MHz, CD $\left.3 \mathrm{OD}\right) \delta 13.8\left(\mathrm{CH}_{3}, 5-\mathrm{CH}_{2}-\mathrm{CH}_{2}-\mathrm{CH}_{2}-\mathrm{CH}_{3}\right), 22.6\left(\mathrm{CH}_{2}\right.$, $\left.4-\mathrm{CH}_{2}-\mathrm{CH}_{2}-\mathrm{N}\right), 23.0\left(\mathrm{CH}_{2}\right), 23.2\left(\mathrm{CH}_{2}\right) \quad\left(5-\mathrm{CH}_{2}-\mathrm{CH}_{2}-\mathrm{CH}_{2}-\mathrm{CH}_{3}\right), 31.6 \quad\left(\mathrm{CH}_{2}\right.$,

5- $\left.\mathrm{CH}_{2}-\mathrm{CH}_{2}-\mathrm{CH}_{2}-\mathrm{CH}_{3}\right), 33.8\left(\mathrm{CH}_{3}, \mathrm{~N}-\mathrm{CH}_{3}\right), 49.4\left(\mathrm{CH}_{2}, 4-\mathrm{CH}_{2}-\mathrm{CH}_{2}-\mathrm{N}\right), 126.8$ 
(2CH), 130.9 (2CH), $131.3(\mathrm{CH})(\mathrm{Ph}-\mathrm{CH}), 137.5$ (C, Ph-C1), 137.7 (C, C5), 141.2 (C, C4); HRMS (ESI), calcd for $\left(\mathrm{C}_{15} \mathrm{H}_{22} \mathrm{~N}_{4}+\mathrm{H}^{+}\right) 259.1917$, found 259.1922 .

\subsubsection{3. $N$-[(1-Ethyl-5-methyl-1H-1,2,3-triazol-4-yl)methyl]-N-methyl-N-propargylamine} 51

It was prepared as described for 31. From amine $44(128 \mathrm{mg}, 0.83 \mathrm{mmol}), \mathrm{Cs}_{2} \mathrm{CO}_{3}(270$ $\mathrm{mg}, 0.83 \mathrm{mmol})$, and propargyl bromide $(80 \%$ solution in toluene, $0.12 \mathrm{~mL}, 0.81$ $\mathrm{mmol})$, and stirring at $0{ }^{\circ} \mathrm{C}$ for $2 \mathrm{~h}$, propargylamine $\mathbf{5 1}(96 \mathrm{mg}, 62 \%$ yield) was obtained as a yellow oil, without the need of chromatographic purification; $R_{f} 0.43\left(\mathrm{CH}_{2} \mathrm{Cl}_{2} /\right.$ $\mathrm{MeOH} / 50 \%$ aq. $\mathrm{NH}_{4} \mathrm{OH}$ 9:1:0.05).

51·HCl: yellow sticky solid, IR (ATR) v 3500-2300 (max at 3386, 3197, 2938, 2496, 2352, ${ }^{+} \mathrm{N}-\mathrm{H}, \equiv \mathrm{C}-\mathrm{H}$, and $\left.\mathrm{C}-\mathrm{H}\right), 2123(\mathrm{C} \equiv \mathrm{C}) \mathrm{cm}^{-1} ;{ }^{1} \mathrm{H}$ NMR (400 MHz, CD $\left.{ }_{3} \mathrm{OD}\right) \delta 1.50$ $\left(\mathrm{t}, J=7.2 \mathrm{~Hz}, 3 \mathrm{H}, 1-\mathrm{CH}_{2}-\mathrm{CH}_{3}\right), 2.45\left(\mathrm{~s}, 3 \mathrm{H}, 5-\mathrm{CH}_{3}\right), 3.00\left(\mathrm{~s}, 3 \mathrm{H}, \mathrm{N}-\mathrm{CH}_{3}\right), 3.44(\mathrm{t}, J=$ $2.4 \mathrm{~Hz}, 1 \mathrm{H}$, propargyl $\mathrm{CH}), 4.17\left(\mathrm{~d}, J=2.4 \mathrm{~Hz}, 2 \mathrm{H}\right.$, propargyl $\left.\mathrm{CH}_{2}\right), 4.39$ (q, $J=7.2$ $\left.\mathrm{Hz}, 2 \mathrm{H}, 1-\mathrm{CH}_{2}-\mathrm{CH}_{3}\right), 4.52$ (s, 2H, 4- $\left.\mathrm{CH}_{2}-\mathrm{N}\right), 4.84\left(\mathrm{~s},{ }^{+} \mathrm{NH}\right) ;{ }^{13} \mathrm{C} \mathrm{NMR}(100.6 \mathrm{MHz}$, $\left.\mathrm{CD}_{3} \mathrm{OD}\right) \delta 8.0\left(\mathrm{CH}_{3}, 5-\mathrm{CH}_{3}\right), 15.2\left(\mathrm{CH}_{3}, 1-\mathrm{CH}_{2}-\mathrm{CH}_{3}\right), 40.3\left(\mathrm{CH}_{3}, \mathrm{~N}-\mathrm{CH}_{3}\right), 44.5\left(\mathrm{CH}_{2}\right.$, 1- $\left.\mathrm{CH}_{2}-\mathrm{CH}_{3}\right), 45.6\left(\mathrm{CH}_{2}\right.$, propargyl $\left.\mathrm{CH}_{2}\right), 49.8\left(\mathrm{CH}_{2}, 4-\mathrm{CH}_{2}-\mathrm{N}\right), 73.1(\mathrm{C}$, propargyl C), 81.5 (CH, propargyl $\mathrm{CH}), 135.1$ (C, C5), 136.3 (C, C4); HRMS (ESI), calcd for $\left(\mathrm{C}_{10} \mathrm{H}_{16} \mathrm{~N}_{4}+\mathrm{H}^{+}\right)$193.1448, found 193.1450.

Note: From amine 44 (117 mg, $0.76 \mathrm{mmol}), \mathrm{Cs}_{2} \mathrm{CO}_{3}(362 \mathrm{mg}, 1.11 \mathrm{mmol})$, and propargyl bromide ( $80 \%$ solution in toluene, $0.16 \mathrm{~mL}, 1.08 \mathrm{mmol})$, and stirring at $\mathrm{rt}$ overnight, an oily residue was obtained and purified through column chromatography (40-60 $\mu \mathrm{m}$ silica gel, $\mathrm{CH}_{2} \mathrm{Cl}_{2} / \mathrm{MeOH} / 50 \%$ aq. $\mathrm{NH}_{4} \mathrm{OH}$ mixtures, gradient elution). On elution with $\mathrm{CH}_{2} \mathrm{Cl}_{2} / \mathrm{MeOH} / 50 \%$ aq. $\mathrm{NH}_{4} \mathrm{OH}$ 99:1:0.2 to 98:2:0.2, propargylamine $\mathbf{5 1}$ (15 mg, 10\% yield) was isolated as a colorless oil. On elution with $\mathrm{CH}_{2} \mathrm{Cl}_{2} / \mathrm{MeOH} / 50 \%$ aq. $\mathrm{NH}_{4} \mathrm{OH}$ 98:2:0.2, slightly impure $\mathrm{N}$-[(1-ethyl-5-methyl- $\mathrm{H}$ 1,2,3-triazol-4-yl)methyl]- $N$-methyl- $N, N$-dipropargylammonium bromide (223 $\mathrm{mg}$ ) was isolated as a brown oil; $R_{f} 0.04\left(\mathrm{CH}_{2} \mathrm{Cl}_{2} / \mathrm{MeOH} / 50 \%\right.$ aq. $\mathrm{NH}_{4} \mathrm{OH}$ 9:1:0.05).

A solution of the dipropargylated byproduct $(223 \mathrm{mg})$ in $\mathrm{CH}_{2} \mathrm{Cl}_{2}(20 \mathrm{~mL})$ was filtered through a PTFE filter $(0.2 \mu \mathrm{m})$ and evaporated under reduced pressure. The resulting residue was washed with pentane $(3 \times 2 \mathrm{~mL})$ to give, after drying under standard conditions, the analytical sample of the dipropargylated byproduct $(213 \mathrm{mg})$ as a brownish oil; IR (ATR) v $3207(\equiv \mathrm{C}-\mathrm{H}), 2124$ (C三C st) cm ${ }^{-1} ;{ }^{1} \mathrm{H}$ NMR (400 MHz, 
$\left.\mathrm{CDCl}_{3}\right) \delta 1.53\left(\mathrm{t}, J=7.6 \mathrm{~Hz}, 3 \mathrm{H}, 1-\mathrm{CH}_{2}-\mathrm{CH}_{3}\right), 2.73\left(\mathrm{~s}, 3 \mathrm{H}, 5-\mathrm{CH}_{3}\right), 2.85(\mathrm{t}, J=2.4$ $\mathrm{Hz}, 2 \mathrm{H}, 2$ propargyl $\mathrm{CH}), 3.42\left(\mathrm{~s}, 3 \mathrm{H}, \mathrm{N}-\mathrm{CH}_{3}\right), 4.34\left(\mathrm{q}, J=7.6 \mathrm{~Hz}, 2 \mathrm{H}, 1-\mathrm{CH}_{2}-\mathrm{CH}_{3}\right)$, $4.83\left(\mathrm{dd}, J=16.0 \mathrm{~Hz}, J^{\prime}=2.4 \mathrm{~Hz}, 2 \mathrm{H}\right), 4.91\left(\mathrm{dd}, J=16.0 \mathrm{~Hz}, J^{\prime}=2.4 \mathrm{~Hz}, 2 \mathrm{H}\right)(2$ propargyl $\left.\mathrm{CH}_{2}\right), 5.34\left(\mathrm{~s}, 2 \mathrm{H}, 4-\mathrm{CH}_{2}-\mathrm{N}\right) ;{ }^{13} \mathrm{C} \mathrm{NMR}\left(100.6 \mathrm{MHz}, \mathrm{CDCl}_{3}\right) \delta 8.8\left(\mathrm{CH}_{3}\right.$, 5- $\left.\mathrm{CH}_{3}\right), 14.8\left(\mathrm{CH}_{3}, 1-\mathrm{CH}_{2}-\mathrm{CH}_{3}\right), 43.6\left(\mathrm{CH}_{2}, 1-\mathrm{CH}_{2}-\mathrm{CH}_{3}\right), 47.2\left(\mathrm{CH}_{3}, \mathrm{~N}-\mathrm{CH}_{3}\right), 51.8$ $\left(2 \mathrm{CH}_{2}, 2\right.$ propargyl $\left.\mathrm{CH}_{2}\right), 55.7\left(\mathrm{CH}_{2}, 4-\mathrm{CH}_{2}-\mathrm{N}\right), 70.9(2 \mathrm{C}, 2$ propargyl $\mathrm{C}), 82.2(2 \mathrm{CH}$, 2 propargyl $\mathrm{CH}$ ), 132.5 (C, C5), $136.5(\mathrm{C}, \mathrm{C} 4)$; HRMS (ESI), calcd for $\left(\mathrm{C}_{13} \mathrm{H}_{19} \mathrm{~N}_{4}{ }^{+}\right)$ 231.1604, found 231.1613.

\subsubsection{N-[2-(1-Ethyl-5-methyl-1H-1,2,3-triazol-4-yl)ethyl]-N-methyl-N-}

\section{propargylamine 52}

It was prepared as described for 31. From amine 45 (490 mg, $2.91 \mathrm{mmol}), \mathrm{Cs}_{2} \mathrm{CO}_{3}(952$ $\mathrm{mg}, 2.92 \mathrm{mmol})$, and propargyl bromide ( $80 \%$ solution in toluene, $0.43 \mathrm{~mL}, 2.89$ mmol), and stirring at $0{ }^{\circ} \mathrm{C}$ for $2.5 \mathrm{~h}$, propargylamine 52 (319 $\mathrm{mg}, 54 \%$ yield) was obtained as a yellow oil, without the need of chromatographic purification; $R_{f} 0.59$ $\left(\mathrm{CH}_{2} \mathrm{Cl}_{2} / \mathrm{MeOH} / 50 \%\right.$ aq. $\mathrm{NH}_{4} \mathrm{OH}$ 9:1:0.05).

52. HCl: yellow sticky solid, IR (ATR) v 3500-2300 (max at 3406, 3181, 2981, 2932, 2583, 2518, 2461, 2408, 2366, ${ }^{+} \mathrm{N}-\mathrm{H}, \equiv \mathrm{C}-\mathrm{H}$, and $\left.\mathrm{C}-\mathrm{H}\right), 2123(\mathrm{C} \equiv \mathrm{C}) \mathrm{cm}^{-1} ;{ }^{1} \mathrm{H}$ NMR $\left(400 \mathrm{MHz}, \mathrm{CD}_{3} \mathrm{OD}\right) \delta 1.56\left(\mathrm{t}, J=7.2 \mathrm{~Hz}, 3 \mathrm{H}, 1-\mathrm{CH}_{2}-\mathrm{CH}_{3}\right), 2.50\left(\mathrm{~s}, 3 \mathrm{H}, 5-\mathrm{CH}_{3}\right), 3.08$ (s, $\left.3 \mathrm{H}, \mathrm{N}-\mathrm{CH}_{3}\right), 3.35$ (t, $\left.J=7.6 \mathrm{~Hz}, 2 \mathrm{H}, 4-\mathrm{CH}_{2}-\mathrm{CH}_{2}-\mathrm{N}\right), 3.45$ (t, $J=2.8 \mathrm{~Hz}, 1 \mathrm{H}$, propargyl $\mathrm{CH}$ ), $3.63\left(\mathrm{t}, J=7.6 \mathrm{~Hz}, 2 \mathrm{H}, 4-\mathrm{CH}_{2}-\mathrm{CH}_{2}-\mathrm{N}\right), 4.28(\mathrm{~d}, J=2.8 \mathrm{~Hz}, 2 \mathrm{H}$, propargyl $\left.\mathrm{CH}_{2}\right), 4.51$ (q, $\left.J=7.2 \mathrm{~Hz}, 2 \mathrm{H}, 1-\mathrm{CH}_{2}-\mathrm{CH}_{3}\right), 4.94\left(\mathrm{~s},{ }^{+} \mathrm{NH}\right) ;{ }^{13} \mathrm{C}$ NMR $(100.6$ $\left.\mathrm{MHz}, \quad \mathrm{CD}_{3} \mathrm{OD}\right) \quad \delta \quad 8.0 \quad\left(\mathrm{CH}_{3}, \quad 5-\mathrm{CH}_{3}\right), \quad 14.4 \quad\left(\mathrm{CH}_{3}, \quad 1-\mathrm{CH}_{2}-\mathrm{CH}_{3}\right), 20.0 \quad\left(\mathrm{CH}_{2}\right.$, $\left.4-\mathrm{CH}_{2}-\mathrm{CH}_{2}-\mathrm{N}\right), 40.7\left(\mathrm{CH}_{3}, \mathrm{~N}-\mathrm{CH}_{3}\right), 46.3\left(\mathrm{CH}_{2}\right.$, propargyl $\left.\mathrm{CH}_{2}\right), 46.4\left(\mathrm{CH}_{2}\right.$, $\left.1-\mathrm{CH}_{2}-\mathrm{CH}_{3}\right), 54.0\left(\mathrm{CH}_{2}, 4-\mathrm{CH}_{2}-\mathrm{CH}_{2}-\mathrm{N}\right), 72.5(\mathrm{C}$, propargyl C), $81.9(\mathrm{CH}$, propargyl $\mathrm{CH}), 136.3$ (C, C5), 137.8 (C, C4); HRMS (ESI), calcd for $\left(\mathrm{C}_{11} \mathrm{H}_{18} \mathrm{~N}_{4}+\mathrm{H}^{+}\right)$207.1604, found 207.1609.

\subsubsection{5. $N$-Methyl-N-\{2-[5-methyl-1-( $\alpha$-methyl)benzyl-1H-1,2,3-triazol-4-yl]ethyl\}-N-} propargylamine 53

It was prepared as described for 31. From amine 46 (234 mg, $0.93 \mathrm{mmol}), \mathrm{Cs}_{2} \mathrm{CO}_{3}(0.30$ g, $0.92 \mathrm{mmol}$ ), and propargyl bromide ( $80 \%$ solution in toluene, $0.10 \mathrm{~mL}, 0.67 \mathrm{mmol}$ ), and stirring at at $0{ }^{\circ} \mathrm{C}$ for $30 \mathrm{~min}$ and at $\mathrm{rt}$ for an additional $3 \mathrm{~h}$, an orange oily residue 
(263 mg) was obtained and purified through column chromatography (40-60 $\mu \mathrm{m}$ silica gel, $\mathrm{CH}_{2} \mathrm{Cl}_{2} / \mathrm{MeOH} / 50 \%$ aq. $\mathrm{NH}_{4} \mathrm{OH}$ mixtures, gradient elution). On elution with $\mathrm{CH}_{2} \mathrm{Cl}_{2} / \mathrm{MeOH} / 50 \%$ aq. $\mathrm{NH}_{4} \mathrm{OH}$ 99.97:0.03:0.2, propargylamine 53 (100 mg, 53\% yield) was obtained as a yellow oil; $R_{f} 0.45\left(\mathrm{CH}_{2} \mathrm{Cl}_{2} / \mathrm{MeOH} / 50 \%\right.$ aq. $\mathrm{NH}_{4} \mathrm{OH}$ 9:1:0.05).

53. $\mathrm{HCl}$ : beige sticky solid, IR (ATR) v 3400-2300 (max at 3386, 3209, 2936, 2351, ${ }^{+} \mathrm{N}-\mathrm{H}, \equiv \mathrm{C}-\mathrm{H}$, and $\left.\mathrm{C}-\mathrm{H}\right), 2118(\mathrm{C} \equiv \mathrm{C}) \mathrm{cm}^{-1} ;{ }^{1} \mathrm{H}$ NMR $\left(400 \mathrm{MHz}, \mathrm{CD}_{3} \mathrm{OD}\right) \delta 2.01[\mathrm{~d}, J=$ $\left.6.8 \mathrm{~Hz}, 3 \mathrm{H}, 1-\mathrm{CH}\left(\mathrm{CH}_{3}\right)-\mathrm{Ph}\right], 2.26\left(\mathrm{~s}, 3 \mathrm{H}, 5-\mathrm{CH}_{3}\right), 3.06\left(\mathrm{~s}, 3 \mathrm{H}, \mathrm{N}-\mathrm{CH}_{3}\right), 3.22(\mathrm{t}, J=8.0$ $\left.\mathrm{Hz}, 2 \mathrm{H}, 4-\mathrm{CH}_{2}-\mathrm{CH}_{2}-\mathrm{N}\right), 3.42$ (t, $J=2.4 \mathrm{~Hz}, 1 \mathrm{H}$, propargyl CH), 3.59 (t, $J=8.0 \mathrm{~Hz}$, $\left.2 \mathrm{H}, 4-\mathrm{CH}_{2}-\mathrm{CH}_{2}-\mathrm{N}\right), 4.25\left(\mathrm{~d}, J=2.4 \mathrm{~Hz}, 2 \mathrm{H}, \operatorname{propargyl} \mathrm{CH}_{2}\right), 4.89\left(\mathrm{~s},{ }^{+} \mathrm{NH}\right), 5.86[\mathrm{q}, J$ $\left.=6.8 \mathrm{~Hz}, 1 \mathrm{H}, 1-\mathrm{CH}\left(\mathrm{CH}_{3}\right)-\mathrm{Ph}\right], 7.24-7.40$ (complex signal, $\left.5 \mathrm{H}, \mathrm{Ph}-\mathrm{CH}\right) ;{ }^{13} \mathrm{C} \mathrm{NMR}$ $\left(100.6 \mathrm{MHz}, \mathrm{CD}_{3} \mathrm{OD}\right) \delta 8.1\left(\mathrm{CH}_{3}, 5-\mathrm{CH}_{3}\right), 20.5\left(\mathrm{CH}_{2}, 4-\mathrm{CH}_{2}-\mathrm{CH}_{2}-\mathrm{N}\right), 22.0\left[\mathrm{CH}_{3}\right.$, 1- $\left.\mathrm{CH}\left(\mathrm{CH}_{3}\right)-\mathrm{Ph}\right], 40.7\left(\mathrm{CH}_{3}, \quad \mathrm{~N}-\mathrm{CH}_{3}\right), 46.3\left(\mathrm{CH}_{2}\right.$, propargyl $\left.\mathrm{CH}_{2}\right), 54.7\left(\mathrm{CH}_{2}\right.$, 4- $\left.\mathrm{CH}_{2}-\mathrm{CH}_{2}-\mathrm{N}\right), 61.0\left[\mathrm{CH}, 1-\mathrm{CH}\left(\mathrm{CH}_{3}\right)-\mathrm{Ph}\right], 72.6$ (C, propargyl C), $81.7(\mathrm{CH}$, propargyl $\mathrm{CH}), 127.4(2 \mathrm{CH}), 129.6(\mathrm{CH}), 130.2(2 \mathrm{CH})(\mathrm{Ph}-\mathrm{CH}), 134.5(\mathrm{C}, \mathrm{Ph}-\mathrm{C} 1)$, 139.6 (C, C5), 141.1 (C, C4); HRMS (ESI), calcd for $\left(\mathrm{C}_{17} \mathrm{H}_{22} \mathrm{~N}_{4}+\mathrm{H}^{+}\right)$283.1917, found 283.1923.

4.1.46. N-[(5-Butyl-1-methyl-1H-1,2,3-triazol-4-yl)methyl]-N-methyl-N-propargylamine 54

It was prepared as described for 31. From amine $47(529 \mathrm{mg}, 2.90 \mathrm{mmol}), \mathrm{Cs}_{2} \mathrm{CO}_{3}(946$ $\mathrm{mg}, 2.90 \mathrm{mmol})$, and propargyl bromide $(80 \%$ solution in toluene, $0.44 \mathrm{~mL}, 2.96$ $\mathrm{mmol}$ ), and stirring at $\mathrm{rt}$ for $3.5 \mathrm{~h}$, propargylamine $\mathbf{5 4}$ (418 $\mathrm{mg}, 65 \%$ yield) was obtained as a yellow oil, without the need of chromatographic purification; $R_{f} 0.29\left(\mathrm{CH}_{2} \mathrm{Cl}_{2} /\right.$ $\mathrm{MeOH} / 50 \%$ aq. $\mathrm{NH}_{4} \mathrm{OH}$ 9.5:0.5:0.05).

54 $\cdot \mathrm{HCl}$ : brown sticky solid, IR (ATR) v 3500-2400 (max at 3411, 3219, 2955, 2930, 2863, 2491, ${ }^{+} \mathrm{N}-\mathrm{H}, \equiv \mathrm{C}-\mathrm{H}$, and $\left.\mathrm{C}-\mathrm{H}\right), 2121(\mathrm{C} \equiv \mathrm{C}) \mathrm{cm}^{-1} ;{ }^{1} \mathrm{H}$ NMR (400 MHz, CD $\left.{ }_{3} \mathrm{OD}\right)$ $\delta 0.98\left(\mathrm{t}, J=7.6 \mathrm{~Hz}, 3 \mathrm{H}, 5-\mathrm{CH}_{2}-\mathrm{CH}_{2}-\mathrm{CH}_{2}-\mathrm{CH}_{3}\right), 1.43$ (tq, $J=J^{\prime}=7.6 \mathrm{~Hz}, 2 \mathrm{H}$, $\left.5-\mathrm{CH}_{2}-\mathrm{CH}_{2}-\mathrm{CH}_{2}-\mathrm{CH}_{3}\right), 1.59\left(\mathrm{tt}, J=J^{\prime}=7.6 \mathrm{~Hz}, 2 \mathrm{H}, 5-\mathrm{CH}_{2}-\mathrm{CH}_{2}-\mathrm{CH}_{2}-\mathrm{CH}_{3}\right), 2.88$ (t, $\left.J=7.6 \mathrm{~Hz}, 2 \mathrm{H}, 5-\mathrm{CH}_{2}-\mathrm{CH}_{2}-\mathrm{CH}_{2}-\mathrm{CH}_{3}\right), 3.03\left(\mathrm{~s}, 3 \mathrm{H}, \mathrm{N}-\mathrm{CH}_{3}\right), 3.47(\mathrm{t}, J=2.4 \mathrm{~Hz}, 1 \mathrm{H}$, propargyl $\mathrm{CH}), 4.06\left(\mathrm{~s}, 3 \mathrm{H}, 1-\mathrm{CH}_{3}\right), 4.22\left(\mathrm{~d}, J=2.4 \mathrm{~Hz}, 2 \mathrm{H}\right.$, propargyl $\left.\mathrm{CH}_{2}\right), 4.55(\mathrm{~s}$, $\left.2 \mathrm{H},{ }_{4}-\mathrm{CH}_{2}-\mathrm{N}\right), 4.92\left(\mathrm{~s},{ }^{+} \mathrm{NH}\right) ;{ }^{13} \mathrm{C} \mathrm{NMR}\left(100.6 \mathrm{MHz}, \mathrm{CD}_{3} \mathrm{OD}\right) \delta 14.1\left(\mathrm{CH}_{3}\right.$, 
5- $\left.\mathrm{CH}_{2}-\mathrm{CH}_{2}-\mathrm{CH}_{2}-\mathrm{CH}_{3}\right), 23.0\left(\mathrm{CH}_{2}\right), 23.4\left(\mathrm{CH}_{2}\right)\left(5-\mathrm{CH}_{2}-\mathrm{CH}_{2}-\mathrm{CH}_{2}-\mathrm{CH}_{3}\right), 31.6\left(\mathrm{CH}_{2}\right.$, 5- $\left.\mathrm{CH}_{2}-\mathrm{CH}_{2}-\mathrm{CH}_{2}-\mathrm{CH}_{3}\right), 35.5\left(\mathrm{CH}_{3}, 1-\mathrm{CH}_{3}\right), 40.5\left(\mathrm{CH}_{3}, \mathrm{~N}-\mathrm{CH}_{3}\right), 45.6\left(\mathrm{CH}_{2}\right.$, propargyl $\left.\mathrm{CH}_{2}\right), 49.5\left(\mathrm{CH}_{2}, 4-\mathrm{CH}_{2}-\mathrm{N}\right), 73.2(\mathrm{C}$, propargyl $\mathrm{C}), 81.6(\mathrm{CH}$, propargyl $\mathrm{CH}), 134.9$ (C, C5), 140.5 (C, C4); HRMS (ESI), calcd for $\left(\mathrm{C}_{12} \mathrm{H}_{20} \mathrm{~N}_{4}+\mathrm{H}^{+}\right)$221.1761, found 221.1762 .

\subsubsection{N-Methyl-N-[2-(5-ethyl-1-phenyl-1H-1,2,3-triazol-4-yl)ethyl]-N-} propargylamine 55

It was prepared as described for 31. From amine 48 (260 mg, $1.13 \mathrm{mmol}), \mathrm{Cs}_{2} \mathrm{CO}_{3}(0.37$ g, $1.14 \mathrm{mmol}$ ), and propargyl bromide ( $80 \%$ solution in toluene, $0.13 \mathrm{~mL}, 0.87 \mathrm{mmol})$, and stirring at $0{ }^{\circ} \mathrm{C}$ for $30 \mathrm{~min}$ and at $\mathrm{rt}$ for an additional $3 \mathrm{~h}$, an orange oil (333 $\mathrm{mg}$ ) was obtained and purified through column chromatography (40-60 $\mu \mathrm{m}$ silica gel, $\mathrm{CH}_{2} \mathrm{Cl}_{2}$ / $\mathrm{MeOH} / 50 \%$ aq. $\mathrm{NH}_{4} \mathrm{OH}$ mixtures, gradient elution). On elution with $\mathrm{CH}_{2} \mathrm{Cl}_{2} / \mathrm{MeOH} /$ 50\% aq. $\mathrm{NH}_{4} \mathrm{OH}$ 99.9:0.1:0.2, propargylamine 55 (222 $\mathrm{mg}$, 95\% yield) was obtained as a yellow oil; $R_{f} 0.59\left(\mathrm{CH}_{2} \mathrm{Cl}_{2} / \mathrm{MeOH} / 50 \%\right.$ aq. $\mathrm{NH}_{4} \mathrm{OH}$ 9:1:0.05).

55 $\mathrm{HCl}$ : yellow sticky solid, IR (ATR) $v$ 3400-2300 (max at 3395, 3193, 3059, 2958, 2925, 2573, 2518, 2459, 2397, ${ }^{+} \mathrm{N}-\mathrm{H}, \equiv \mathrm{C}-\mathrm{H}$, and $\left.\mathrm{C}-\mathrm{H}\right), 2123(\mathrm{C} \equiv \mathrm{C}) \mathrm{cm}^{-1} ;{ }^{1} \mathrm{H}$ NMR $\left(400 \mathrm{MHz}, \mathrm{CD}_{3} \mathrm{OD}\right) \delta 1.06\left(\mathrm{t}, J=7.6 \mathrm{~Hz}, 3 \mathrm{H}, 5-\mathrm{CH}_{2}-\mathrm{CH}_{3}\right), 2.81(\mathrm{q}, J=7.6 \mathrm{~Hz}, 2 \mathrm{H}$, 5- $\left.\mathrm{CH}_{2}-\mathrm{CH}_{3}\right), 3.11\left(\mathrm{~s}, 3 \mathrm{H}, \mathrm{N}-\mathrm{CH}_{3}\right), 3.27$ (t, $\left.J=7.6 \mathrm{~Hz}, 2 \mathrm{H}, 4-\mathrm{CH}_{2}-\mathrm{CH}_{2}-\mathrm{N}\right), 3.45$ (t, $J$ $=2.8 \mathrm{~Hz}, 1 \mathrm{H}$, propargyl $\mathrm{CH}), 3.70\left(\mathrm{~m}, 2 \mathrm{H}, 4-\mathrm{CH}_{2}-\mathrm{CH}_{2}-\mathrm{N}\right), 4.31(\mathrm{~d}, J=2.8 \mathrm{~Hz}, 2 \mathrm{H}$, propargyl $\left.\mathrm{CH}_{2}\right), 4.88\left(\mathrm{~s},{ }^{\dagger} \mathrm{NH}\right), 7.53[\mathrm{ddm}, J=J '=7.6 \mathrm{~Hz}, 2 \mathrm{H}, \mathrm{Ph}-\mathrm{C} 2(6)-H]$, 7.62-7.67 [complex signal, 3H, Ph-C3(5)-H, Ph-C4-H]; ${ }^{13} \mathrm{C}$ NMR (100.6 MHz, $\left.\mathrm{CD}_{3} \mathrm{OD}\right) \quad \delta \quad 13.4\left(\mathrm{CH}_{3}, \quad 5-\mathrm{CH}_{2}-\mathrm{CH}_{3}\right), 16.9\left(\mathrm{CH}_{2}, 5-\mathrm{CH}_{2}-\mathrm{CH}_{3}\right), \quad 21.1 \quad\left(\mathrm{CH}_{2}\right.$, 4- $\left.\mathrm{CH}_{2}-\mathrm{CH}_{2}-\mathrm{N}\right), 40.8\left(\mathrm{CH}_{3}, \mathrm{~N}-\mathrm{CH}_{3}\right), 46.3\left(\mathrm{CH}_{2}\right.$, propargyl $\left.\mathrm{CH}_{2}\right), 55.3\left(\mathrm{CH}_{2}\right.$, 4- $\left.\mathrm{CH}_{2}-\mathrm{CH}_{2}-\mathrm{N}\right), 72.7$ (C, propargyl C), $81.7(\mathrm{CH}$, propargyl $\mathrm{CH}), 126.7(2 \mathrm{CH}), 130.9$ (2CH), $131.4(\mathrm{CH})(\mathrm{Ph}-\mathrm{CH}), 137.6$ (C, Ph-C1), 139.1 (C, C5), 140.2 (C, C4); HRMS (ESI), calcd for $\left(\mathrm{C}_{16} \mathrm{H}_{20} \mathrm{~N}_{4}+\mathrm{H}^{+}\right)$269.1761; found 269.1766 .

\subsubsection{N-Methyl-N-[2-(1-phenyl-5-propyl-1H-1,2,3-triazol-4-yl)ethyl]-N-}

\section{propargylamine 56}

It was prepared as described for 31. From amine 49 (168 mg, $0.69 \mathrm{mmol}), \mathrm{Cs}_{2} \mathrm{CO}_{3}(0.22$ $\mathrm{g}, 0.68 \mathrm{mmol})$, and propargyl bromide ( $80 \%$ solution in toluene, $0.07 \mathrm{~mL}, 0.47 \mathrm{mmol}$ ), and stirring at $0{ }^{\circ} \mathrm{C}$ for $30 \mathrm{~min}$ and at $\mathrm{rt}$ for an additional $3 \mathrm{~h}$, an oily residue $(278 \mathrm{mg}$ ) 
was obtained and purified through column chromatography $(40-60 \mu \mathrm{m}$ silica gel, $\mathrm{CH}_{2} \mathrm{Cl}_{2} / \mathrm{MeOH} / 50 \%$ aq. $\mathrm{NH}_{4} \mathrm{OH}$ mixtures, gradient elution). On elution with $\mathrm{CH}_{2} \mathrm{Cl}_{2}$ / $\mathrm{MeOH} / 50 \%$ aq. $\mathrm{NH}_{4} \mathrm{OH}$ 98:2:0.2, propargylamine 56 (117 mg, 88\% yield) was obtained as a yellow oil; $R_{f} 0.84\left(\mathrm{CH}_{2} \mathrm{Cl}_{2} / \mathrm{MeOH} / 50 \%\right.$ aq. $\mathrm{NH}_{4} \mathrm{OH}$ 9:1:0.05).

56. $\mathrm{HCl}$ : orange sticky solid, IR (ATR) v 3500-2300 (max at 3400, 3197, 2958, 2930, 2873, 2354, ${ }^{+} \mathrm{N}-\mathrm{H}, \equiv \mathrm{C}-\mathrm{H}$, and $\left.\mathrm{C}-\mathrm{H}\right), 2121(\mathrm{C} \equiv \mathrm{C}) \mathrm{cm}^{-1} ;{ }^{1} \mathrm{H}$ NMR (400 MHz, $\left.\mathrm{CD}_{3} \mathrm{OD}\right)$ $\delta 0.83\left(\mathrm{t}, J=7.6 \mathrm{~Hz}, 3 \mathrm{H}, 5-\mathrm{CH}_{2}-\mathrm{CH}_{2}-\mathrm{CH}_{3}\right), 1.44$ (tq, $J=J^{\prime}=7.6 \mathrm{~Hz}, 2 \mathrm{H}$, 5- $\mathrm{CH}_{2}-\mathrm{CH}_{2}-\mathrm{CH}_{3}$ ), 2.77 (t, $\left.J=7.6 \mathrm{~Hz}, 2 \mathrm{H}, 5-\mathrm{CH}_{2}-\mathrm{CH}_{2}-\mathrm{CH}_{3}\right), 3.11$ (s, 3H, N-CH ), $3.22\left(\mathrm{t}, J=7.6 \mathrm{~Hz}, 2 \mathrm{H}, 4-\mathrm{CH}_{2}-\mathrm{CH}_{2}-\mathrm{N}\right), 3.45$ (t, $J=2.4 \mathrm{~Hz}, 1 \mathrm{H}$, propargyl CH), 3.70 $\left(\mathrm{m}, 2 \mathrm{H}, 4-\mathrm{CH}_{2}-\mathrm{CH}_{2}-\mathrm{N}\right), 4.31\left(\mathrm{~d}, J=2.4 \mathrm{~Hz}, 2 \mathrm{H}\right.$, propargyl $\left.\mathrm{CH}_{2}\right), 4.85\left(\mathrm{~s},{ }^{+} \mathrm{NH}\right), 7.52$ (m, 2H), 7.62-7.66 (complex signal, 3H) (Ph-CH); ${ }^{13} \mathrm{C}$ NMR (100.6 MHz, $\left.\mathrm{CD}_{3} \mathrm{OD}\right) \delta$ $13.9\left(\mathrm{CH}_{3}, \quad 5-\mathrm{CH}_{2}-\mathrm{CH}_{2}-\mathrm{CH}_{3}\right), \quad 21.1 \quad\left(\mathrm{CH}_{2}, \quad 4-\mathrm{CH}_{2}-\mathrm{CH}_{2}-\mathrm{N}\right), \quad 22.8 \quad\left(\mathrm{CH}_{2}\right.$, 5- $\left.\mathrm{CH}_{2}-\mathrm{CH}_{2}-\mathrm{CH}_{3}\right), 25.2\left(\mathrm{CH}_{2}, 5-\mathrm{CH}_{2}-\mathrm{CH}_{2}-\mathrm{CH}_{3}\right), 40.9\left(\mathrm{CH}_{3}, \mathrm{~N}-\mathrm{CH}_{3}\right), 46.3\left(\mathrm{CH}_{2}\right.$, propargyl $\left.\mathrm{CH}_{2}\right), 55.3\left(\mathrm{CH}_{2}, 4-\mathrm{CH}_{2}-\mathrm{CH}_{2}-\mathrm{N}\right), 72.7(\mathrm{C}$, propargyl $\mathrm{C}), 81.7(\mathrm{CH}$, propargyl $\mathrm{CH}), 126.8(2 \mathrm{CH}), 130.9(2 \mathrm{CH}), 131.4(\mathrm{CH})(\mathrm{Ph}-\mathrm{CH}), 137.6(\mathrm{C}), 137.7(\mathrm{C})$ (C5, Ph-C1), 140.7 (C, C4); HRMS (ESI), calcd for $\left(\mathrm{C}_{17} \mathrm{H}_{22} \mathrm{~N}_{4}+\mathrm{H}^{+}\right)$283.1917, found 283.1923.

\subsubsection{N-Methyl-N-[2-(5-butyl-1-phenyl-1H-1,2,3-triazol-4-yl)ethyl]-N-}

propargylamine 57

It was prepared as described for 31. From amine 50 (157 mg, $0.61 \mathrm{mmol}), \mathrm{Cs}_{2} \mathrm{CO}_{3}(0.22$ $\mathrm{g}, 0.68 \mathrm{mmol}$ ), and propargyl bromide ( $80 \%$ solution in toluene, $0.07 \mathrm{~mL}, 0.47 \mathrm{mmol})$, and stirring at $0{ }^{\circ} \mathrm{C}$ for $30 \mathrm{~min}$ and at $\mathrm{rt}$ for an additional $3 \mathrm{~h}$, an oily residue (172 $\mathrm{mg}$ ) was obtained and purified through column chromatography $(40-60 \mu \mathrm{m}$ silica gel, $\mathrm{CH}_{2} \mathrm{Cl}_{2} / \mathrm{MeOH} / 50 \%$ aq. $\mathrm{NH}_{4} \mathrm{OH}$ mixtures, gradient elution). On elution with $\mathrm{CH}_{2} \mathrm{Cl}_{2}$ / $\mathrm{MeOH} / 50 \%$ aq. $\mathrm{NH}_{4} \mathrm{OH}$ 100:0:0.2 to 99:1:0.2, propargylamine 57 (164 mg, 91\% yield) was obtained as a yellow oil; $R_{f} 0.86\left(\mathrm{CH}_{2} \mathrm{Cl}_{2} / \mathrm{MeOH} / 50 \%\right.$ aq. $\mathrm{NH}_{4} \mathrm{OH}$ 9:1:0.05).

57. HCl: yellow sticky solid, IR (ATR) v 3500-2300 (max at 3426, 3209, 2962, 2929, 2869, 2352, ${ }^{+} \mathrm{N}-\mathrm{H}, \equiv \mathrm{C}-\mathrm{H}$, and $\left.\mathrm{C}-\mathrm{H}\right), 2120(\mathrm{C} \equiv \mathrm{C}) \mathrm{cm}^{-1} ;{ }^{1} \mathrm{H}$ NMR (400 MHz, $\left.\mathrm{CD}_{3} \mathrm{OD}\right)$ $\delta 0.80\left(\mathrm{t}, J=7.6 \mathrm{~Hz}, 3 \mathrm{H}, 5-\mathrm{CH}_{2}-\mathrm{CH}_{2}-\mathrm{CH}_{2}-\mathrm{CH}_{3}\right), 1.23$ (tq, $J=J^{\prime}=7.6 \mathrm{~Hz}, 2 \mathrm{H}$, 5- $\left.\mathrm{CH}_{2}-\mathrm{CH}_{2}-\mathrm{CH}_{2}-\mathrm{CH}_{3}\right), 1.39\left(\mathrm{tt}, J=J^{\prime}=7.6 \mathrm{~Hz}, 2 \mathrm{H}, 5-\mathrm{CH}_{2}-\mathrm{CH}_{2}-\mathrm{CH}_{2}-\mathrm{CH}_{3}\right), 2.78$ (t, 
$\left.J=7.6 \mathrm{~Hz}, 2 \mathrm{H}, 5-\mathrm{CH}_{2}-\mathrm{CH}_{2}-\mathrm{CH}_{2}-\mathrm{CH}_{3}\right), 3.11\left(\mathrm{~s}, 3 \mathrm{H}, \mathrm{N}-\mathrm{CH}_{3}\right), 3.22(\mathrm{t}, J=7.6 \mathrm{~Hz}, 2 \mathrm{H}$, $\left.4-\mathrm{CH}_{2}-\mathrm{CH}_{2}-\mathrm{N}\right), 3.46(\mathrm{t}, \mathrm{J}=2.4 \mathrm{~Hz}, 1 \mathrm{H}$, propargyl $\mathrm{CH}), 3.69\left(\mathrm{~m}, 2 \mathrm{H}, 4-\mathrm{CH}_{2}-\mathrm{CH}_{2}-\mathrm{N}\right)$, $4.30\left(\mathrm{~d}, J=2.4 \mathrm{~Hz}, 2 \mathrm{H}\right.$, propargyl $\left.\mathrm{CH}_{2}\right), 4.85\left(\mathrm{~s},{ }^{+} \mathrm{NH}\right), 7.50(\mathrm{~m}, 2 \mathrm{H}), 7.62-7.66$ (complex signal, 2H) $(\mathrm{Ph}-\mathrm{CH}) ;{ }^{13} \mathrm{C} \mathrm{NMR}\left(100.6 \mathrm{MHz}, \mathrm{CD}_{3} \mathrm{OD}\right) \delta 13.8\left(\mathrm{CH}_{3}\right.$, 5- $\left.\mathrm{CH}_{2}-\mathrm{CH}_{2}-\mathrm{CH}_{2}-\mathrm{CH}_{3}\right), 21.1\left(\mathrm{CH}_{2}, \quad 4-\mathrm{CH}_{2}-\mathrm{CH}_{2}-\mathrm{N}\right), 23.1 \quad\left(\mathrm{CH}_{2}\right), 23.2\left(\mathrm{CH}_{2}\right)$ (5- $\left.\mathrm{CH}_{2}-\mathrm{CH}_{2}-\mathrm{CH}_{2}-\mathrm{CH}_{3}\right), 31.6\left(\mathrm{CH}_{2}, 5-\mathrm{CH}_{2}-\mathrm{CH}_{2}-\mathrm{CH}_{2}-\mathrm{CH}_{3}\right), 40.9\left(\mathrm{CH}_{3}, \mathrm{~N}-\mathrm{CH}_{3}\right)$, $46.3\left(\mathrm{CH}_{2}\right.$, propargyl $\left.\mathrm{CH}_{2}\right), 55.3\left(\mathrm{CH}_{2}, 4-\mathrm{CH}_{2}-\mathrm{CH}_{2}-\mathrm{N}\right), 72.7(\mathrm{C}$, propargyl $\mathrm{C}), 81.7$ (CH, propargyl $\mathrm{CH}), 126.8(2 \mathrm{CH}), 130.9(2 \mathrm{CH}), 131.4(\mathrm{CH})(\mathrm{Ph}-\mathrm{CH}), 137.69(\mathrm{C})$, 137.72 (C) (C5, Ph-C1), 140.6 (C, C4); HRMS (ESI), calcd for $\left(\mathrm{C}_{18} \mathrm{H}_{24} \mathrm{~N}_{4}+\mathrm{H}^{+}\right)$ 297.2074, found 297.2085.

\subsubsection{3-[3-(Trimethylsilyl)-2-propynyl] benzonitrile 61}

A solution of trimethylsilylacetylene $(1.59 \mathrm{~mL}, 1.11 \mathrm{~g}, 11.3 \mathrm{mmol})$ in anhydrous THF $(22 \mathrm{~mL})$ was cooled to $-78{ }^{\circ} \mathrm{C}$, treated dropwise with $n$-BuLi $(2.5 \mathrm{M}$ solution in hexanes, $4.59 \mathrm{~mL}, 11.5 \mathrm{mmol}$ ), and stirred at $-78{ }^{\circ} \mathrm{C}$ for $30 \mathrm{~min}$. A solution of $\mathrm{ZnBr}_{2}$ $(2.59 \mathrm{~g}, 11.5 \mathrm{mmol})$ in anhydrous THF $(8 \mathrm{~mL})$ was then added and the resulting mixture was warmed to $0{ }^{\circ} \mathrm{C}$. 3-Bromomethylbenzonitrile, $58(1.50 \mathrm{~g}, 7.65 \mathrm{mmol})$, and $\mathrm{Pd}\left(\mathrm{PPh}_{3}\right)_{2} \mathrm{Cl}_{2}(290 \mathrm{mg}, 0.41 \mathrm{mmol})$ were added and the reaction mixture was stirred at $\mathrm{rt}$ overnight. The reaction mixture was cooled to $0{ }^{\circ} \mathrm{C}$, treated with $1 \mathrm{~N} \mathrm{HCl}(20 \mathrm{~mL})$, and extracted with EtOAc $(2 \times 30 \mathrm{~mL})$. The combined organic extracts were washed with sat. aq. $\mathrm{NaHCO}_{3}(20 \mathrm{~mL})$, dried over anhydrous $\mathrm{Na}_{2} \mathrm{SO}_{4}$, and evaporated under reduced pressure to give a residue ( $2.02 \mathrm{~g})$, which was purified through column chromatography (40-60 $\mu \mathrm{m}$ silica gel, hexane / EtOAc mixtures, gradient elution). On elution with hexane / EtOAc 95:5, compound 61 (1.03 g, 63\% yield) was isolated as a colorless oil; $R_{f} 0.73$ (hexane / EtOAc 8:2); $2230(\mathrm{C} \equiv \mathrm{N}), 2178(\mathrm{C} \equiv \mathrm{C}) \mathrm{cm}^{-1} ;{ }^{1} \mathrm{H}$ NMR (400 MHz, $\left.\mathrm{CDCl}_{3}\right) \delta 0.20\left[\mathrm{~s}, 9 \mathrm{H}, \mathrm{Si}\left(\mathrm{CH}_{3}\right)_{3}\right], 3.68\left(\mathrm{~s}, 2 \mathrm{H}, 1^{\prime}-\mathrm{H}_{2}\right), 7.42\left(\mathrm{dd}, J=J^{\prime}=7.6 \mathrm{~Hz}, 1 \mathrm{H}, 5-\right.$ H), 7.53 (d, $J=7.6 \mathrm{~Hz}, 1 \mathrm{H}), 7.57$ (d, $J=7.6 \mathrm{~Hz}, 1 \mathrm{H})(4-\mathrm{H}, 6-\mathrm{H}), 7.65$ (br s, 1H, 2-H); ${ }^{13} \mathrm{C}$ NMR $\left(100.6 \mathrm{MHz}, \mathrm{CDCl}_{3}\right) \delta 0.2\left[3 \mathrm{CH}_{3}, \mathrm{Si}\left(\mathrm{CH}_{3}\right)_{3}\right], 26.0\left(\mathrm{CH}_{2}, \mathrm{Cl}{ }^{\prime}\right), 88.6\left(\mathrm{C}, \mathrm{C} 3{ }^{\prime}\right)$, $102.4(\mathrm{C}, \mathrm{C} 2$ '), $112.7(\mathrm{C}, \mathrm{C} 1), 118.9(\mathrm{C}, \mathrm{CN}), 129.4(\mathrm{CH}), 130.6(\mathrm{CH}), 131.6(\mathrm{CH})$, $132.5(\mathrm{CH})$ (phenylene $\mathrm{CH}), 138.1(\mathrm{C}, \mathrm{C} 3)$; HRMS (ESI), calcd for $\left(\mathrm{C}_{13} \mathrm{H}_{15} \mathrm{NSi}+\mathrm{H}^{+}\right)$ 214.1047, found 214.1048.

\subsubsection{4-[3-(Trimethylsilyl)-2-propynyl] benzonitrile 62}


It was prepared as described for 61. From trimethylsilylacetylene $(1.06 \mathrm{~mL}, 737 \mathrm{mg}$, $7.50 \mathrm{mmol}), n$-BuLi (2.5 M solution in hexanes, $3.06 \mathrm{~mL}, 7.65 \mathrm{mmol}), \mathrm{ZnBr}_{2}(1.72 \mathrm{~g}$, $7.64 \mathrm{mmol}$ ), 4-bromomethylbenzonitrile, 59 (1.00 g, $5.10 \mathrm{mmol})$, and $\mathrm{Pd}\left(\mathrm{PPh}_{3}\right)_{2} \mathrm{Cl}_{2}(183$ $\mathrm{mg}, 0.26 \mathrm{mmol})$, a residue (1.38 g) was obtained and purified through column chromatography (40-60 $\mu \mathrm{m}$ silica gel, hexane / EtOAc mixtures, gradient elution). On elution with hexane / EtOAc 90:10, compound 62 (900 mg, 83\% yield) was isolated as a colorless oil; $R_{f} 0.77$ (hexane / EtOAc 8:2); IR (ATR) v $2230(\mathrm{C} \equiv \mathrm{N}), 2178(\mathrm{C} \equiv \mathrm{C})$ $\mathrm{cm}^{-1} ;{ }^{1} \mathrm{H} \mathrm{NMR}\left(400 \mathrm{MHz}, \mathrm{CDCl}_{3}\right) \delta 0.19\left[\mathrm{~s}, 9 \mathrm{H}, \mathrm{Si}\left(\mathrm{CH}_{3}\right)_{3}\right], 3.70\left(\mathrm{~s}, 2 \mathrm{H}, 1\right.$ '- $\left.\mathrm{H}_{2}\right), 7.46$ $[\mathrm{dm}, J=8.4 \mathrm{~Hz}, 2 \mathrm{H}, 3(5)-\mathrm{H}], 7.61[\mathrm{dm}, J=8.4 \mathrm{~Hz}, 2 \mathrm{H}, 2(6)-\mathrm{H}] ;{ }^{13} \mathrm{C}$ NMR $(100.6 \mathrm{MHz}$, $\left.\mathrm{CDCl}_{3}\right) \delta 0.1\left[3 \mathrm{CH}_{3}, \mathrm{Si}\left(\mathrm{CH}_{3}\right)_{3}\right], 26.5\left(\mathrm{CH}_{2}, \mathrm{C}^{\prime}\right.$ '), $88.6(\mathrm{C}, \mathrm{C} 3$ ') $102.3(\mathrm{C}, \mathrm{C} 2$ '), 110.8 (C, C1), 119.0 (C, CN), 128.8 [2CH, C3(5)], 132.5 [2CH, C2(6)], 142.1 (C, C4); HRMS (ESI), calcd for $\left(\mathrm{C}_{13} \mathrm{H}_{15} \mathrm{NSi}+\mathrm{H}^{+}\right)$214.1047; found 214.1048 .

\subsubsection{4-[3-(Trimethylsilyl)-2-propynyl] phenylacetonitrile 63}

It was prepared as described for 61. From trimethylsilylacetylene $(5.20 \mathrm{~mL}, 3.61 \mathrm{~g}, 36.8$ mmol), $n$-BuLi (2.5 M solution in hexanes, $15.0 \mathrm{~mL}, 37.5 \mathrm{mmol}), \mathrm{ZnBr}_{2}(8.46 \mathrm{~g}, 37.6$ mmol), 4-(bromomethyl)phenylacetonitrile, 60 (5.26 g, $25.0 \mathrm{mmol}$ ), and $\mathrm{Pd}\left(\mathrm{PPh}_{3}\right)_{2} \mathrm{Cl}_{2}$ (940 mg, $1.34 \mathrm{mmol})$, a residue (7.12 g) was obtained and purified through column chromatography (40-60 $\mu \mathrm{m}$ silica gel, hexane / EtOAc mixtures, gradient elution). On elution with hexane / EtOAc 95:5, compound 63 (2.50 g, 44\% yield) was isolated as a yellow oil; $R_{f} 0.50$ (hexane / EtOAc 8:2); IR (ATR) $v 2344(\mathrm{C} \equiv \mathrm{N}), 2144(\mathrm{C} \equiv \mathrm{C}) \mathrm{cm}^{-1}$; ${ }^{1} \mathrm{H}$ NMR $\left(400 \mathrm{MHz}, \mathrm{CDCl}_{3}\right) \delta 0.19$ [s, 9H, $\left.\mathrm{Si}\left(\mathrm{CH}_{3}\right)_{3}\right], 3.65$ (s, 2H, 1'- $\left.\mathrm{H}_{2}\right), 3.73(\mathrm{~s}, 2 \mathrm{H}$, $1-\mathrm{CH}_{2}-\mathrm{CN}$ ), 7.28 (br d, $\left.J=8.4 \mathrm{~Hz}, 2 \mathrm{H}\right), 7.36$ (br d, $\left.J=8.4 \mathrm{~Hz}, 2 \mathrm{H}\right)(\mathrm{Ph}-\mathrm{CH}) ;{ }^{13} \mathrm{C}$ NMR $\left(100.6 \mathrm{MHz}, \mathrm{CDCl}_{3}\right) \delta 0.03\left[3 \mathrm{CH}_{3}, \mathrm{Si}\left(\mathrm{CH}_{3}\right)_{3}\right], 23.3\left(\mathrm{CH}_{2}, 1-\mathrm{CH}_{2}-\mathrm{CN}\right), 25.8$ $\left(\mathrm{CH}_{2}, \mathrm{C} 1^{\prime}\right), 87.3\left(\mathrm{C}, \mathrm{C}^{\prime}\right.$ '), $103.6(\mathrm{C}, \mathrm{C} 2$ ') $, 117.8(\mathrm{C}, \mathrm{CN}), 128.1(2 \mathrm{CH}), 128.6(2 \mathrm{CH})$ $(\mathrm{Ph}-\mathrm{CH}), 128.2(\mathrm{C}, \mathrm{Ph}-\mathrm{C} 1), 136.5(\mathrm{C}, \mathrm{Ph}-\mathrm{C} 4)$; HRMS (ESI), calcd for $\left(\mathrm{C}_{14} \mathrm{H}_{17} \mathrm{NSi}+\right.$ $\mathrm{H}^{+}$) 228.1203; found 228.1203.

\subsubsection{3-(2-Propynyl)benzonitrile 64}

To a solution of $61(1.60 \mathrm{~g}, 7.51 \mathrm{mmol})$ in $\mathrm{CH}_{2} \mathrm{Cl}_{2}(70 \mathrm{~mL})$, a solution of AgOTf (193 $\mathrm{mg}, 0.75 \mathrm{mmol})$ in $\mathrm{MeOH} / \mathrm{H}_{2} \mathrm{O}$ 11:1 (50 mL) was added. The reaction mixture was stirred at $\mathrm{rt}$ for $7 \mathrm{~h}$, treated with an additional amount of AgOTf (386 mg, $1.50 \mathrm{mmol}$ ), and stirred at $\mathrm{rt}$ overnight. The resulting mixture was diluted with sat. aq. $\mathrm{NH}_{4} \mathrm{Cl}$ (50 
$\mathrm{mL})$ and extracted with $\mathrm{CH}_{2} \mathrm{Cl}_{2}(3 \times 50 \mathrm{~mL})$. The combined organic extracts were washed with $\mathrm{H}_{2} \mathrm{O}(50 \mathrm{~mL})$, dried over anhydrous $\mathrm{Na}_{2} \mathrm{SO}_{4}$, and evaporated under reduced pressure to give a crude $(944 \mathrm{mg}$ ), which was purified through column chromatography (40-60 $\mu \mathrm{m}$ silica gel, hexane / EtOAc mixtures, gradient elution). On elution with hexane / EtOAc 95:5, alkyne 64 (690 mg, 65\% yield) was isolated as a white solid; $R_{f} 0.72$ (hexane / EtOAc 8:2), mp 63-65 ${ }^{\circ} \mathrm{C}$; IR (ATR) $v 3247$ (三C-H), $2231(\mathrm{C} \equiv \mathrm{N}, \mathrm{C} \equiv \mathrm{C}) \mathrm{cm}^{-1} ;{ }^{1} \mathrm{H}$ NMR $\left(400 \mathrm{MHz}, \mathrm{CDCl}_{3}\right) \delta 2.26\left(\mathrm{t}, J=2.8 \mathrm{~Hz}, 1 \mathrm{H}, 3^{\prime}-\mathrm{H}\right)$, $3.64\left(\mathrm{~d}, J=2.8 \mathrm{~Hz}, 2 \mathrm{H}, 1^{\prime}-\mathrm{H}_{2}\right), 7.43\left(\mathrm{dd}, J=J^{\prime}=8.0 \mathrm{~Hz}, 1 \mathrm{H}, 5-\mathrm{H}\right), 7.54$ (dm, $J=8.0$ $\mathrm{Hz}, 1 \mathrm{H}), 7.58(\mathrm{dm}, J=8.0 \mathrm{~Hz}, 1 \mathrm{H})(4-\mathrm{H}, 6-\mathrm{H}), 7.68(\mathrm{~m}, 1 \mathrm{H}, 2-\mathrm{H}) ;{ }^{13} \mathrm{C}$ NMR $(100.6$ $\left.\mathrm{MHz}, \mathrm{CDCl}_{3}\right) \delta 24.6\left(\mathrm{CH}_{2}, \mathrm{C}^{\prime}\right.$ '), $71.9(\mathrm{CH}, \mathrm{C} 3$ '), 80.3 (C, C2'), $112.8(\mathrm{C}, \mathrm{C} 1), 118.8$ $(\mathrm{C}, \mathrm{CN}), 129.4(\mathrm{CH}), 130.7(\mathrm{CH}), 131.5(\mathrm{CH}), 132.5(\mathrm{CH})$ (phenylene $\mathrm{CH}), 137.7(\mathrm{C}$, C3); HRMS (ESI), calcd for $\left(\mathrm{C}_{10} \mathrm{H}_{7} \mathrm{~N}+\mathrm{H}^{+}\right)$142.0651, found 142.0653 .

\subsubsection{4-(2-Propynyl)benzonitrile 65}

It was prepared as described for 64. From $62(642 \mathrm{mg}, 3.01 \mathrm{mmol})$ and AgOTf [(80 mg, $0.31 \mathrm{mmol}) \times 2]$, a crude $(548 \mathrm{mg})$ was obtained and purified through column chromatography (40-60 $\mu \mathrm{m}$ silica gel, hexane / EtOAc mixtures, gradient elution). On elution with hexane / EtOAc 80:20, alkyne $\mathbf{6 5}$ (346 mg, 81\% yield) was isolated as a white solid; $R_{f} 0.76$ (hexane / EtOAc 8:2), mp 87-89 ${ }^{\circ} \mathrm{C}$; IR (ATR) $v 3254(\equiv \mathrm{C}-\mathrm{H})$, $2230(\mathrm{C} \equiv \mathrm{N}, \mathrm{C} \equiv \mathrm{C}) \mathrm{cm}^{-1} ;{ }^{1} \mathrm{H}$ NMR $\left(400 \mathrm{MHz}, \mathrm{CDCl}_{3}\right) \delta 2.25\left(\mathrm{t}, J=2.8 \mathrm{~Hz}, 1 \mathrm{H}, 3^{\prime}-\mathrm{H}\right)$, $3.67\left(\mathrm{~d}, J=2.8 \mathrm{~Hz}, 2 \mathrm{H}, 1^{\prime}-\mathrm{H}_{2}\right), 7.48$ [dm, $\left.J=8.8 \mathrm{~Hz}, 2 \mathrm{H}, 3(5)-\mathrm{H}\right], 7.62$ [dm, $J=8.8 \mathrm{~Hz}$, 2H, 2(6)-H]; ${ }^{13} \mathrm{C}$ NMR (100.6 MHz, $\left.\mathrm{CDCl}_{3}\right) \delta 25.2\left(\mathrm{CH}_{2}, \mathrm{C} 1^{\prime}\right), 71.9(\mathrm{CH}, \mathrm{C} 3$ '), 80.2 (C, C2'), 111.0 (C, C1), 118.9 (C, CN), 128.8 [2CH, C3(5)], 132.5 [2CH, C2(6)], 141.7 (C, C4); HRMS (ESI), calcd for $\left(\mathrm{C}_{10} \mathrm{H}_{7} \mathrm{~N}+\mathrm{H}^{+}\right)$142.0651, found 142.0647.

\subsubsection{4-(2-Propynyl)phenylacetonitrile $\mathbf{6 6}$}

It was prepared as described for 64. From 63 (2.50 g, $11.0 \mathrm{mmol})$ and $\operatorname{AgOTf}(280 \mathrm{mg}$, $1.09 \mathrm{mmol}) \times 2$ ], a crude $(1.79 \mathrm{~g})$ was obtained and purified through column chromatography (40-60 $\mu \mathrm{m}$ silica gel, hexane / EtOAc mixtures, gradient elution). On elution with hexane / EtOAc 95:5, alkyne 66 (1.31 g, 77\% yield) was isolated as a white solid; $R_{f} 0.41$ (hexane / EtOAc 8:2), mp 58-59 ${ }^{\circ} \mathrm{C}$; IR (ATR) v $3262(\equiv \mathrm{C}-\mathrm{H}), 2254$ $(\mathrm{C} \equiv \mathrm{N}, \mathrm{C} \equiv \mathrm{C}) \mathrm{cm}^{-1} ;{ }^{1} \mathrm{H}$ NMR (400 MHz, $\left.\mathrm{CDCl}_{3}\right) \delta 2.19\left(\mathrm{t}, J=2.8 \mathrm{~Hz}, 1 \mathrm{H}, 3{ }^{\prime}-\mathrm{H}\right), 3.57$ (br s, 2H, 1'- $\mathrm{H}_{2}$ ), 3.69 (s, 2H, 1- $\left.\mathrm{CH}_{2}-\mathrm{CN}\right), 7.27$ (br d, $\left.J=8.0 \mathrm{~Hz}, 2 \mathrm{H}\right), 7.36$ (br d, $J=$ 
8.0 Hz, 2H) $(\mathrm{Ph}-\mathrm{CH}) ;{ }^{13} \mathrm{C}$ NMR (100.6 MHz, $\left.\mathrm{CDCl}_{3}\right) \delta 23.2\left(\mathrm{CH}_{2}, 1-\mathrm{CH}_{2}-\mathrm{CN}\right), 24.4$ $\left(\mathrm{CH}_{2}, \mathrm{C}^{\prime}\right.$ '), $70.8(\mathrm{CH}, \mathrm{C} 3$ '), $81.4(\mathrm{C}, \mathrm{C} 2$ ') $117.8(\mathrm{C}, \mathrm{CN}), 128.1(2 \mathrm{CH}), 128.6(2 \mathrm{CH})$ $(\mathrm{Ph}-\mathrm{CH}), 128.3(\mathrm{C}, \mathrm{Ph}-\mathrm{C} 1), 136.1(\mathrm{C}, \mathrm{Ph}-\mathrm{C} 4)$; HRMS (ESI), calcd for $\left(\mathrm{C}_{11} \mathrm{H}_{9} \mathrm{~N}+\mathrm{H}^{+}\right)$ 156.0808 , found 156.0808 .

\subsubsection{3-[(1-Methyl-1H-1,2,3-triazol-4-yl)methyl]benzonitrile 67}

It was prepared as described for 9. From iodomethane $(0.30 \mathrm{~mL}, 0.68 \mathrm{~g}, 4.82 \mathrm{mmol})$, $\mathrm{NaN}_{3}$ (342 mg, $\left.5.26 \mathrm{mmol}\right), \mathrm{Na}_{2} \mathrm{CO}_{3}(1.39 \mathrm{~g}, 13.1 \mathrm{mmol}$ ), ascorbic acid (616 mg, 3.50 mmol), $\mathrm{CuSO}_{4} \cdot 5 \mathrm{H}_{2} \mathrm{O}(436 \mathrm{mg}, 1.75 \mathrm{mmol})$, and alkyne $64(618 \mathrm{mg}, 4.38 \mathrm{mmol})$, compound 67 ( $823 \mathrm{mg}, 95 \%$ yield) was obtained as a yellowish solid; $R_{f} 0.64\left(\mathrm{CH}_{2} \mathrm{Cl}_{2}\right.$ / $\mathrm{MeOH} / 50 \%$ aq. $\mathrm{NH}_{4} \mathrm{OH}$ 9.5:0.5:0.05); mp 85-87 ${ }^{\circ} \mathrm{C}$; IR (ATR) $v 2229(\mathrm{C} \equiv \mathrm{N}) \mathrm{cm}^{-1}$; ${ }^{1} \mathrm{H}$ NMR (400 MHz, $\left.\mathrm{CDCl}_{3}\right) \delta 4.05$ (s, 3H, 1'- $\mathrm{CH}_{3}$ ), 4.09 (br s, 2H, 3- $\mathrm{CH}_{2}$ ), 7.25 (s, 1H, 5'-H), 7.39 (dd, $\left.J=J^{\prime}=8.0 \mathrm{~Hz}, 1 \mathrm{H}, 5-\mathrm{H}\right), 7.48-7.53$ (complex signal, 3H, 2-H, 4$\mathrm{H}, 6-\mathrm{H}) ;{ }^{13} \mathrm{C} \mathrm{NMR}\left(100.6 \mathrm{MHz}, \mathrm{CDCl}_{3}\right) \delta 31.7\left(\mathrm{CH}_{3}, 3-\mathrm{CH}_{3}\right), 36.8\left(\mathrm{CH}_{2}, 1{ }^{\prime}-\mathrm{CH}_{2}\right)$, $112.7(\mathrm{C}, \mathrm{C} 1), 118.8(\mathrm{C}, \mathrm{CN}), 122.6(\mathrm{CH}, \mathrm{C} 5$ '), $129.5(\mathrm{CH}), 130.4(\mathrm{CH}), 132.2(\mathrm{CH})$, $133.4(\mathrm{CH})$ (phenylene $\mathrm{CH}), 140.7$ (C, C3), 146.1 (C, C4'); HRMS (ESI), calcd for $\left(\mathrm{C}_{11} \mathrm{H}_{10} \mathrm{~N}_{4}+\mathrm{H}^{+}\right)$199.0978, found 199.0980 .

\subsubsection{4-[(1-Methyl-1H-1,2,3-triazol-4-yl)methyl]benzonitrile 68}

It was prepared as described for 9. From iodomethane $(0.17 \mathrm{~mL}, 0.38 \mathrm{~g}, 2.70 \mathrm{mmol})$, $\mathrm{NaN}_{3}$ (191 mg, $\left.2.94 \mathrm{mmol}\right), \mathrm{Na}_{2} \mathrm{CO}_{3}(779 \mathrm{mg}, 7.35 \mathrm{mmol}$ ), ascorbic acid (350 mg, 1.99 $\mathrm{mmol}), \mathrm{CuSO}_{4} \cdot 5 \mathrm{H}_{2} \mathrm{O}(250 \mathrm{mg}, 1.00 \mathrm{mmol})$, and alkyne 65 (346 mg, $\left.2.45 \mathrm{mmol}\right)$, compound 68 (459 mg, 95\% yield) was obtained as a white solid; $R_{f} 0.63\left(\mathrm{CH}_{2} \mathrm{Cl}_{2} /\right.$ $\mathrm{MeOH} / 50 \%$ aq. $\mathrm{NH}_{4} \mathrm{OH}$ 9.5:0.5:0.05); mp 116-118 ${ }^{\circ} \mathrm{C}$; IR (ATR) $v 2227(\mathrm{C} \equiv \mathrm{N}) \mathrm{cm}^{-1}$; ${ }^{1} \mathrm{H}$ NMR (400 MHz, $\left.\mathrm{CDCl}_{3}\right) \delta 4.05$ (s, 3H, 1'- $\mathrm{CH}_{3}$ ), 4.12 (br s, 2H, 4- $\mathrm{CH}_{2}$ ), 7.23 (s, $\left.1 \mathrm{H}, 5^{\prime}-\mathrm{H}\right), 7.36$ [dm, $\left.J=8.4 \mathrm{~Hz}, 2 \mathrm{H}, 3(5)-\mathrm{H}\right], 7.57$ [dm, $\left.J=8.4 \mathrm{~Hz}, 2 \mathrm{H}, 2(6)-\mathrm{H}\right] ;{ }^{13} \mathrm{C}$ NMR (100.6 MHz, $\left.\mathrm{CDCl}_{3}\right) \delta 32.3\left(\mathrm{CH}_{2}, 4-\mathrm{CH}_{2}\right), 36.8\left(\mathrm{CH}_{3}, 1^{\prime}-\mathrm{CH}_{3}\right), 110.6(\mathrm{C}, \mathrm{C} 1)$, $118.9(\mathrm{C}, \mathrm{CN}), 122.6(\mathrm{CH}, \mathrm{C5}$ '), 129.6 [2CH, C3(5)], 132.5 [2CH, C2(6)], $144.8(\mathrm{C}$, C4), 146.1 (C, C4'); HRMS (ESI), calcd for $\left(\mathrm{C}_{11} \mathrm{H}_{10} \mathrm{~N}_{4}+\mathrm{H}^{+}\right)$199.0978, found 199.0978 .

\subsubsection{4-[(1-Methyl-1H-1,2,3-triazol-4-yl)methyl]phenylacetonitrile 69}


It was prepared as described for 9. From iodomethane $(0.58 \mathrm{~mL}, 1.32 \mathrm{~g}, 9.32 \mathrm{mmol})$, $\mathrm{NaN}_{3}(660 \mathrm{mg}, 10.2 \mathrm{mmol}), \mathrm{Na}_{2} \mathrm{CO}_{3}(2.69 \mathrm{~g}, 25.4 \mathrm{mmol})$, ascorbic acid (1.19 g, 6.76 $\mathrm{mmol}), \mathrm{CuSO}_{4} \cdot 5 \mathrm{H}_{2} \mathrm{O}(840 \mathrm{mg}, 3.36 \mathrm{mmol})$, and alkyne $66(1.31 \mathrm{~g}, 8.45 \mathrm{mmol})$, compound 69 (1.60 g, 89\% yield) was obtained as a yellowish solid; $R_{f} 0.93\left(\mathrm{CH}_{2} \mathrm{Cl}_{2} /\right.$ $\mathrm{MeOH} / 50 \%$ aq. $\mathrm{NH}_{4} \mathrm{OH}$ 9.5:0.5:0.05); mp 86-88 ${ }^{\circ} \mathrm{C}$; IR (ATR) $v 2241(\mathrm{C} \equiv \mathrm{N}) \mathrm{cm}^{-1}$; ${ }^{1} \mathrm{H}$ NMR (400 MHz, $\left.\mathrm{CDCl}_{3}\right) \delta 3.71\left(\mathrm{~s}, 2 \mathrm{H}, 1-\mathrm{CH}_{2}-\mathrm{CN}\right), 4.02$ (s, 3H, 1'- $\mathrm{CH}_{3}$ ), 4.06 (s, $\left.2 \mathrm{H}, 4-\mathrm{CH}_{2}\right), 7.21(\mathrm{~s}, 1 \mathrm{H}, 5$ '-H), 7.23-7.30 (complex signal, $4 \mathrm{H}, \mathrm{Ph}-\mathrm{CH}) ;{ }^{13} \mathrm{C} \mathrm{NMR}$ $\left(100.6 \mathrm{MHz}, \mathrm{CDCl}_{3}\right) \delta 23.1\left(\mathrm{CH}_{2}, 1-\mathrm{CH}_{2}-\mathrm{CN}\right), 31.6\left(\mathrm{CH}_{2}, 4-\mathrm{CH}_{2}\right), 36.4\left(\mathrm{CH}_{3}\right.$, 1'- $\left.\mathrm{CH}_{3}\right), 117.8(\mathrm{C}, \mathrm{CN}), 122.3(\mathrm{CH}, \mathrm{C} 5$ '), 128.0 (C, Ph-C1), $128.1(2 \mathrm{CH}), 129.2$ $(2 \mathrm{CH})(\mathrm{Ph}-\mathrm{CH}), 139.0(\mathrm{C}, \mathrm{Ph}-\mathrm{C} 4), 147.1\left(\mathrm{C}, \mathrm{C}^{\prime}\right)$; HRMS (ESI), calcd for $\left(\mathrm{C}_{12} \mathrm{H}_{12} \mathrm{~N}_{4}+\right.$ $\mathrm{H}^{+}$) 213.1135, found 213.1135.

\subsubsection{3-[(1-Methyl-1H-1,2,3-triazol-4-yl)methyl]benzylamine 70}

A solution of nitrile $67(739 \mathrm{mg}, 3.73 \mathrm{mmol})$ in anhydrous THF $(30 \mathrm{~mL})$ was cooled to $0{ }^{\circ} \mathrm{C}$ and treated dropwise with $\mathrm{LiAlH}_{4}\left(4 \mathrm{M}\right.$ solution in $\mathrm{Et}_{2} \mathrm{O}, 2.98 \mathrm{~mL}, 11.9 \mathrm{mmol}$ ). The reaction mixture was stirred under reflux for $2 \mathrm{~h}$, cooled to $0{ }^{\circ} \mathrm{C}$, treated portionwise with $1 \mathrm{~N} \mathrm{NaOH}(100 \mathrm{~mL})$, diluted with $\mathrm{H}_{2} \mathrm{O}(100 \mathrm{~mL})$, and extracted with EtOAc $(3 \times 100 \mathrm{~mL})$. The combined organic extracts were dried over anhydrous $\mathrm{Na}_{2} \mathrm{SO}_{4}$ and concentrated under reduced pressure to afford amine 70 (368 mg, 49\% yield) as a yellow oil; $R_{f} 0.28\left(\mathrm{CH}_{2} \mathrm{Cl}_{2} / \mathrm{MeOH} / 50 \%\right.$ aq. $\mathrm{NH}_{4} \mathrm{OH}$ 9.5:0.5:0.05).

70. $\mathrm{HCl}$ : yellowish solid, mp $119-121^{\circ} \mathrm{C}$; IR (ATR) v 3400-2300 (max at 3303, 3077, 2908, 2870, 2356, 2322, ${ }^{+} \mathrm{N}-\mathrm{H}$ and $\left.\mathrm{C}-\mathrm{H}\right) \mathrm{cm}^{-1}$; ${ }^{1} \mathrm{H}$ NMR (400 MHz, $\left.\mathrm{CD}_{3} \mathrm{OD}\right) \delta 4.14(\mathrm{~s}$, $\left.2 \mathrm{H}, 3-\mathrm{CH}_{2}\right), 4.296$ (s, 3H, 1'- $\left.\mathrm{CH}_{3}\right), 4.302\left(\mathrm{~s}, 2 \mathrm{H}, 1-\mathrm{CH}_{2}-\mathrm{N}\right), 4.95\left(\mathrm{~s},{ }^{+} \mathrm{NH}_{3}\right), 7.38-7.52$ (complex signal, 4H, phenylene $\mathrm{CH}$ ), 8.39 (s, 1H, 5'-H); ${ }^{13} \mathrm{C}$ NMR (100.6 MHz, $\left.\mathrm{CD}_{3} \mathrm{OD}\right) \delta 30.2\left(\mathrm{CH}_{2}, 3-\mathrm{CH}_{2}\right), 39.8\left(\mathrm{CH}_{3}, 1{ }^{\prime}-\mathrm{CH}_{3}\right), 44.1\left(\mathrm{CH}_{2}, 1-\mathrm{CH}_{2}-\mathrm{N}\right), 129.1(\mathrm{CH})$, $129.2(\mathrm{CH}), 130.7(2 \mathrm{CH}), 131.0(\mathrm{CH})$ (phenylene $\mathrm{CH}, \mathrm{C} 5$ '), $135.3(\mathrm{C}), 138.2(\mathrm{C})(\mathrm{C} 1$, C3), 144.9 (C, C4'); HRMS (ESI), calcd for $\left(\mathrm{C}_{11} \mathrm{H}_{14} \mathrm{~N}_{4}+\mathrm{H}^{+}\right)$203.1291, found 203.1297.

\subsubsection{4-[(1-Methyl-1H-1,2,3-triazol-4-yl)methyl]benzylamine 71}

It was prepared as described for 70. From nitrile $68(310 \mathrm{mg}, 1.56 \mathrm{mmol})$ and $\mathrm{LiAlH}_{4}(4$ $\mathrm{M}$ solution in $\left.\mathrm{Et}_{2} \mathrm{O}, 1.18 \mathrm{~mL}, 4.72 \mathrm{mmol}\right)$, amine 71 (254 $\mathrm{mg}, 81 \%$ yield) was obtained as a yellow oil; $R_{f} 0.27\left(\mathrm{CH}_{2} \mathrm{Cl}_{2} / \mathrm{MeOH} / 50 \%\right.$ aq. $\mathrm{NH}_{4} \mathrm{OH}$ 9.5:0.5:0.05). 
71· $\mathrm{HCl}$ : yellowish sticky solid, IR (ATR) v 3400-2500 ( $\max$ at 3386, 2931, 2810, 2536, ${ }^{+} \mathrm{N}-\mathrm{H}$ and $\left.\mathrm{C}-\mathrm{H}\right) \mathrm{cm}^{-1} ;{ }^{1} \mathrm{H}$ NMR (400 MHz, $\left.\mathrm{CD}_{3} \mathrm{OD}\right) \delta 4.11\left(\mathrm{~s}, 2 \mathrm{H}, 4-\mathrm{CH}_{2}\right), 4.22$ (s, 3H, 1'- $\left.\mathrm{CH}_{3}\right), 4.90\left(\mathrm{~s}, 1-\mathrm{CH}_{2}-\mathrm{N},{ }^{+} \mathrm{NH}_{3}\right), 7.38(\mathrm{~d}, J=8.0 \mathrm{~Hz}, 2 \mathrm{H}), 7.46(\mathrm{~d}, J=8.0$ $\mathrm{Hz}, 2 \mathrm{H})$ (phenylene $\mathrm{CH}), 8.16$ (s, $\left.1 \mathrm{H}, 5{ }^{\prime}-\mathrm{H}\right) ;{ }^{13} \mathrm{C} \mathrm{NMR}\left(100.6 \mathrm{MHz}, \mathrm{CD}_{3} \mathrm{OD}\right) \delta 30.6$ $\left(\mathrm{CH}_{2}, 4-\mathrm{CH}_{2}\right), 38.9\left(\mathrm{CH}_{3}, 1{ }^{\prime}-\mathrm{CH}_{3}\right), 43.9\left(\mathrm{CH}_{2}, 1-\mathrm{CH}_{2}-\mathrm{N}\right), 127.7(\mathrm{CH}, \mathrm{C} 5$ '), 130.6 $(2 \mathrm{CH}), 130.7(2 \mathrm{CH})$ (phenylene $\mathrm{CH}), 133.4(\mathrm{C}), 139.3$ (C) (C1, C4), 145.8 (C, C4'); HRMS (ESI), calcd for $\left(\mathrm{C}_{11} \mathrm{H}_{14} \mathrm{~N}_{4}+\mathrm{H}^{+}\right)$203.1291, found 203.1292 .

\subsubsection{4-[(1-Methyl-1H-1,2,3-triazol-4-yl)methyl]phenethylamine $\mathbf{7 2}$}

A suspension of nitrile $69(1.60 \mathrm{~g}, 7.54 \mathrm{mmol})$ and Raney-Ni $(2.02 \mathrm{~g}$, wet $)$ in $7 \mathrm{~N}$ methanolic $\mathrm{NH}_{3}(32 \mathrm{~mL})$ was hydrogenated by bubbling $\mathrm{H}_{2}$ overnight. The resulting mixture was filtered and the cake was washed with $\mathrm{MeOH}(2 \times 15 \mathrm{~mL})$. The combined filtrates were concentrated under reduced pressure to afford amine 72 (1.70 g, quantitative yield) as an oily residue; $R_{f} 0.17\left(\mathrm{CH}_{2} \mathrm{Cl}_{2} / \mathrm{MeOH} / 50 \%\right.$ aq. $\mathrm{NH}_{4} \mathrm{OH}$ 9.5:0.5:0.05).

72. $\mathrm{HCl}$ : yellow solid, $\mathrm{mp} 158-160{ }^{\circ} \mathrm{C}$; IR (ATR) v 3400-2500 (max at 3390, 3331, 3254, 2994, 2970, 2727, 2627, 2573, ${ }^{+} \mathrm{N}-\mathrm{H}$ and $\left.\mathrm{C}-\mathrm{H}\right) \mathrm{cm}^{-1} ;{ }^{1} \mathrm{H}$ NMR (400 MHz, $\left.\mathrm{CD}_{3} \mathrm{OD}\right) \delta 2.93\left(\mathrm{t}, J=7.6 \mathrm{~Hz}, 2 \mathrm{H}, 1-\mathrm{CH}_{2}-\mathrm{CH}_{2}-\mathrm{N}\right), 3.15(\mathrm{t}, J=7.6 \mathrm{~Hz}, 2 \mathrm{H}$, $\left.1-\mathrm{CH}_{2}-\mathrm{CH}_{2}-\mathrm{N}\right), 4.04\left(\mathrm{~s}, 2 \mathrm{H}, 4-\mathrm{CH}_{2}\right), 4.07\left(\mathrm{~s}, 3 \mathrm{H}, 1^{\prime}-\mathrm{CH}_{3}\right), 4.86\left(\mathrm{~s},{ }^{+} \mathrm{NH}_{3}\right), 7.18-7.27$ (complex signal, 4H, Ph-CH), $7.73\left(\mathrm{~s}, 1 \mathrm{H}, 5^{\prime}-\mathrm{H}\right) ;{ }^{13} \mathrm{C} \mathrm{NMR}\left(100.6 \mathrm{MHz}, \mathrm{CD}_{3} \mathrm{OD}\right) \delta$ $31.9\left(\mathrm{CH}_{2}, 4-\mathrm{CH}_{2}\right), 34.1\left(\mathrm{CH}_{2}, 1-\mathrm{CH}_{2}-\mathrm{CH}_{2}-\mathrm{N}\right), 37.2\left(\mathrm{CH}_{3}, 1^{\prime}-\mathrm{CH}_{3}\right), 42.0\left(\mathrm{CH}_{2}\right.$, 1- $\left.\mathrm{CH}_{2}-\mathrm{CH}_{2}-\mathrm{N}\right), \quad 125.0\left(\mathrm{CH}, \mathrm{C}^{\prime}\right.$ '), $130.1(2 \mathrm{CH}), 130.3(2 \mathrm{CH})(\mathrm{Ph}-\mathrm{CH}), 136.1(\mathrm{C}$, $\mathrm{Ph}-C 1), 139.3(\mathrm{C}, \mathrm{Ph}-C 4), 148.1\left(\mathrm{C}, \mathrm{C} 4^{\prime}\right)$; HRMS (ESI), calcd for $\left(\mathrm{C}_{12} \mathrm{H}_{16} \mathrm{~N}_{4}+\mathrm{H}^{+}\right)$ 217.1448, found 217.1450.

4.1.62. $\mathrm{N}$-(tert-Butoxycarbonyl)-3-[(1-methyl-1H-1,2,3-triazol-4-yl)methyl] benzylamine 73

To a solution of $70(352 \mathrm{mg}, 1.74 \mathrm{mmol})$ in anhydrous THF $(6 \mathrm{~mL})$, a solution of ditert-butyl dicarbonate $(380 \mathrm{mg}, 1.74 \mathrm{mmol})$ in anhydrous THF $(2 \mathrm{~mL})$ was added at 0 ${ }^{\circ} \mathrm{C}$. The reaction mixture was stirred at $\mathrm{rt}$ for $3 \mathrm{~h}$ and evaporated under reduced pressure. The resulting residue was washed with pentane $(3 \times 10 \mathrm{~mL})$ and dried under vacuum, to afford 73 (520 mg, quantitative yield) as a yellow oil; $R_{f} 0.85\left(\mathrm{CH}_{2} \mathrm{Cl}_{2} / \mathrm{MeOH} / 50 \%\right.$ aq. $\mathrm{NH}_{4} \mathrm{OH}$ 9.5:0.5:0.05); IR (ATR) $v 3341(\mathrm{~N}-\mathrm{H}), 1697(\mathrm{C}=\mathrm{O}) \mathrm{cm}^{-1} ;{ }^{1} \mathrm{H}$ NMR (400 
$\left.\mathrm{MHz}, \mathrm{CDCl}_{3}\right) \delta 1.55\left[\mathrm{~s}, 9 \mathrm{H}, \mathrm{C}\left(\mathrm{CH}_{3}\right)_{3}\right], 4.12\left(\mathrm{~s}, 3 \mathrm{H}, 1\right.$ ' $\left.-\mathrm{CH}_{3}\right), 4.16\left(\mathrm{~s}, 2 \mathrm{H}, 3-\mathrm{CH}_{2}\right), 4.38$ (d, $J=5.6 \mathrm{~Hz}, 2 \mathrm{H}, 1-\mathrm{CH}_{2}-\mathrm{N}$ ), 4.97 (br s, 1H, NHBoc), 7.22-7.27 (complex signal, 3H), 7.33-7.38 (overlapped signal, 2H) (phenylene $\mathrm{CH}, 5$ '-H); ${ }^{13} \mathrm{C}$ NMR (100.6 MHz, $\left.\mathrm{CDCl}_{3}\right) \delta 28.5\left[3 \mathrm{CH}_{3}, \mathrm{C}\left(\mathrm{CH}_{3}\right)_{3}\right], 32.3\left(\mathrm{CH}_{2}, 3-\mathrm{CH}_{2}\right), 36.7\left(\mathrm{CH}_{3}, 1^{\prime}-\mathrm{CH}_{3}\right), 44.7\left(\mathrm{CH}_{2}\right.$, $\left.1-\mathrm{CH}_{2}-\mathrm{N}\right), 79.6\left[\mathrm{C}, \mathrm{C}\left(\mathrm{CH}_{3}\right)_{3}\right], 122.6\left(\mathrm{CH}, \mathrm{C} 5^{\prime}\right), 125.7(\mathrm{CH}), 127.8(2 \mathrm{CH}), 129.0(\mathrm{CH})$ (phenylene $\mathrm{CH}$ ), 139.4 (C), 139.6 (C) (C1, C3), 147.8 (C, C4'), 156.0 (C, NCOO); HRMS (ESI), calcd for $\left(\mathrm{C}_{16} \mathrm{H}_{22} \mathrm{~N}_{4} \mathrm{O}_{2}+\mathrm{H}^{+}\right)$303.1816, found 303.1812 .

\subsubsection{3. $\mathrm{N}$-(tert-Butoxycarbonyl)-4-[(1-methyl-1H-1,2,3-triazol-4-yl)methyl]benzylamine}

74

It was prepared as described for 73. From $71(90 \mathrm{mg}, 0.45 \mathrm{mmol})$ and di-tert-butyl dicarbonate (98 $\mathrm{mg}, 0.45 \mathrm{mmol}), 74$ (133 $\mathrm{mg}$, quantitative yield) was obtained as a beige solid; $R_{f} 0.78\left(\mathrm{CH}_{2} \mathrm{Cl}_{2} / \mathrm{MeOH} / 50 \%\right.$ aq. $\mathrm{NH}_{4} \mathrm{OH}$ 9.5:0.5:0.05).

The analytical sample of $\mathbf{7 4}$ was obtained by filtration of a dichloromethane solution through a PTFE filter $(0.2 \mu \mathrm{m})$, evaporation of the filtrate under reduced pressure and washing of the resulting solid with pentane $(3 \times 2 \mathrm{~mL})$; IR (ATR) $v 3382(\mathrm{~N}-\mathrm{H}), 1690$ $(\mathrm{C}=\mathrm{O}) \mathrm{cm}^{-1} ;{ }^{1} \mathrm{H}$ NMR $\left(400 \mathrm{MHz}, \mathrm{CDCl}_{3}\right) \delta 1.44\left[\mathrm{~s}, 9 \mathrm{H}, \mathrm{C}\left(\mathrm{CH}_{3}\right)_{3}\right], 4.00(\mathrm{~s}, 3 \mathrm{H}$, $\left.1^{\prime}-\mathrm{CH}_{3}\right), 4.04\left(\mathrm{~s}, 2 \mathrm{H}, 4-\mathrm{CH}_{2}\right), 4.26\left(\mathrm{~d}, J=5.6 \mathrm{~Hz}, 2 \mathrm{H}, 1-\mathrm{CH}_{2}-\mathrm{N}\right), 4.86($ br s, $1 \mathrm{H}$,

$\mathrm{N} H \mathrm{Boc}$ ), 7.13 (s, 1H, 5'-H), 7.20 (complex signal, 4H, phenylene $\mathrm{CH}$ ); ${ }^{13} \mathrm{C} \mathrm{NMR}$ $\left(100.6 \mathrm{MHz}, \mathrm{CDCl}_{3}\right) \delta 28.5\left[3 \mathrm{CH}_{3}, \mathrm{C}\left(\mathrm{CH}_{3}\right)_{3}\right], 32.0\left(\mathrm{CH}_{2}, 4-\mathrm{CH}_{2}\right), 36.7\left(\mathrm{CH}_{3}, 1{ }^{\prime}-\mathrm{CH}_{3}\right)$, $44.4\left(\mathrm{CH}_{2}, 1-\mathrm{CH}_{2}-\mathrm{N}\right), 79.6\left[\mathrm{C}, \mathrm{C}\left(\mathrm{CH}_{3}\right)_{3}\right], 122.5\left(\mathrm{CH}, \mathrm{C} 5^{\prime}\right), 127.8(2 \mathrm{CH}), 129.0(2 \mathrm{CH})$ (phenylene $\mathrm{CH}$ ), 137.3 (C), 138.3 (C) (C1, C4), 148.0 (C, C4'), 156.0 (C, NCOO); HRMS (ESI), calcd for $\left(\mathrm{C}_{16} \mathrm{H}_{22} \mathrm{~N}_{4} \mathrm{O}_{2}+\mathrm{H}^{+}\right)$303.1816, found 303.1817 .

4.1.64.

N-(tert-Butoxycarbonyl)-4-[(1-methyl-1H-1,2,3-triazol-4yl)methyl]phenethylamine $\mathbf{7 5}$

It was prepared as described for 73. From $72(1.65 \mathrm{~g}, 7.63 \mathrm{mmol})$ and di-tert-butyl dicarbonate (1.67 g, $7.65 \mathrm{mmol}), 75$ (2.34 g, quantitative yield) was obtained as a solid; $R_{f} 0.91\left(\mathrm{CH}_{2} \mathrm{Cl}_{2} / \mathrm{MeOH} / 50 \%\right.$ aq. $\mathrm{NH}_{4} \mathrm{OH}$ 9.5:0.5:0.05); mp 92-94 ${ }^{\circ} \mathrm{C}$; IR (ATR) $v$ $3358(\mathrm{~N}-\mathrm{H}), 1677(\mathrm{C}=\mathrm{O}) \mathrm{cm}^{-1} ;{ }^{1} \mathrm{H}$ NMR $\left(400 \mathrm{MHz}, \mathrm{CDCl}_{3}\right) \delta 1.42\left[\mathrm{~s}, 9 \mathrm{H}, \mathrm{C}\left(\mathrm{CH}_{3}\right)_{3}\right]$, $2.76\left(\mathrm{t}, J=6.8 \mathrm{~Hz}, 2 \mathrm{H}, 1-\mathrm{CH}_{2}-\mathrm{CH}_{2}-\mathrm{N}\right), 3.35\left(\mathrm{dt}, J=J^{\prime}=6.8 \mathrm{~Hz}, 2 \mathrm{H}, 1-\mathrm{CH}_{2}-\mathrm{CH}_{2}-\mathrm{N}\right)$, 4.02 (s, 3H, 1'- $\left.-\mathrm{CH}_{3}\right), 4.05$ (s, 2H, 4- $\mathrm{CH}_{2}$ ), 4.54 (br s, $1 \mathrm{H}, \mathrm{N} H \mathrm{Boc}$ ), 7.12 (br d, $J=8.0$ $\mathrm{Hz}, 2 \mathrm{H}), 7.19$ (br d, $J=8.0 \mathrm{~Hz}, 2 \mathrm{H})(\mathrm{Ph}-\mathrm{CH}), 7.16\left(\mathrm{~s}, 1 \mathrm{H}, 5{ }^{\prime}-\mathrm{H}\right) ;{ }^{13} \mathrm{C}$ NMR $(100.6$ 


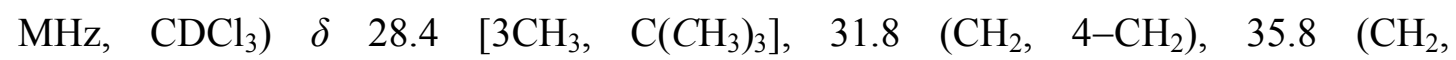
$\left.1-\mathrm{CH}_{2}-\mathrm{CH}_{2}-\mathrm{N}\right), 36.5\left(\mathrm{CH}_{3}, 1^{\prime}-\mathrm{CH}_{3}\right), 41.8\left(\mathrm{CH}_{2}, 1-\mathrm{CH}_{2}-\mathrm{CH}_{2}-\mathrm{N}\right), 79.2\left[\mathrm{C}, \mathrm{C}\left(\mathrm{CH}_{3}\right)_{3}\right]$, $122.3(\mathrm{CH}, \mathrm{C5}$ '), 128.9 (2CH), $129.0(2 \mathrm{CH})(\mathrm{Ph}-\mathrm{CH}), 137.16(\mathrm{C}), 137.23(\mathrm{C})(\mathrm{Ph}-\mathrm{Cl}$, $\mathrm{Ph}-\mathrm{C} 4), 147.9$ (C, C4'), 155.8 (C, NCOO); HRMS (ESI), calcd for $\left(\mathrm{C}_{17} \mathrm{H}_{24} \mathrm{~N}_{4} \mathrm{O}_{2}+\mathrm{H}^{+}\right)$ 317.1972, found 317.1982 .

\subsubsection{N-(tert-Butoxycarbonyl)-N-methyl-3-[(1-methyl-1H-1,2,3-triazol-4- yl)methyl]benzylamine $\mathbf{7 6}$}

It was prepared as described for 17. From compound 73 (480 mg, $1.59 \mathrm{mmol}), \mathrm{NaH}$ (60\% supension in mineral oil, $96 \mathrm{mg}, 2.40 \mathrm{mmol})$, and iodomethane $(0.11 \mathrm{~mL}, 251 \mathrm{mg}$, $1.77 \mathrm{mmol})$, stirring at $60{ }^{\circ} \mathrm{C}$ for $2 \mathrm{~h}$, treating again with $\mathrm{NaH}(60 \%$ supension in mineral oil, $96 \mathrm{mg}, 2.40 \mathrm{mmol})$, and iodomethane $(0.11 \mathrm{~mL}, 251 \mathrm{mg}, 1.77 \mathrm{mmol})$ and stirring at $60{ }^{\circ} \mathrm{C}$ for an additional $2 \mathrm{~h}$, compound 76 (422 $\mathrm{mg}, 84 \%$ yield) was obtained as an orange oil; $R_{f} 0.85\left(\mathrm{CH}_{2} \mathrm{Cl}_{2} / \mathrm{MeOH} / 50 \%\right.$ aq. $\mathrm{NH}_{4} \mathrm{OH}$ 9.5:0.5:0.05); IR (ATR) $v$ $1686(\mathrm{C}=\mathrm{O}) \mathrm{cm}^{-1} ;{ }^{1} \mathrm{H} \mathrm{NMR}\left(400 \mathrm{MHz}, \mathrm{CDCl}_{3}\right) \delta 1.48\left[\mathrm{br} \mathrm{s}, 9 \mathrm{H}, \mathrm{C}\left(\mathrm{CH}_{3}\right)_{3}\right], 2.82(\mathrm{br} \mathrm{s}$, $\left.3 \mathrm{H}, \mathrm{N}-\mathrm{CH}_{3}\right), 4.04\left(\mathrm{~s}, 3 \mathrm{H}, 1\right.$ ' $\left.-\mathrm{CH}_{3}\right), 4.08\left(\mathrm{~s}, 2 \mathrm{H}, 3-\mathrm{CH}_{2}\right), 4.40$ (br s, 2H, 1- $\left.\mathrm{CH}_{2}-\mathrm{N}\right)$, 7.08-7.19 (complex signal), 7.25-7.30 (complex signal) (5H, phenylene $\mathrm{CH}, 5$ '-H); ${ }^{13} \mathrm{C}$ NMR $\left(100.6 \mathrm{MHz}, \mathrm{CDCl}_{3}\right) \delta 28.6\left[3 \mathrm{CH}_{3}, \mathrm{C}\left(\mathrm{CH}_{3}\right)_{3}\right], 32.3\left(\mathrm{CH}_{2}, 3-\mathrm{CH}_{2}\right), 34.1\left(\mathrm{CH}_{3}\right.$, $\left.\mathrm{N}-\mathrm{CH}_{3}\right), 36.7\left(\mathrm{CH}_{3}, 1^{\prime}-\mathrm{CH}_{3}\right), 52.0\left(\right.$ br $\left.\mathrm{CH}_{2}\right), 52.7\left(\right.$ br $\left.\mathrm{CH}_{2}\right)\left(1-\mathrm{CH}_{2}-\mathrm{N}\right), 79.8[\mathrm{C}$, $\left.C\left(\mathrm{CH}_{3}\right)\right], 122.5$ (CH, C5'), 125.6 (br CH), 125.8 (br CH), $127.7(\mathrm{CH}), 127.8$ (br CH), 128.9 (CH) (phenylene CH), 138.6 (C), 139.5 (C) (C1, C3), 147.9 (br C, C4'), 156.4 (br C, NCOO); HRMS (ESI), calcd for $\left(\mathrm{C}_{17} \mathrm{H}_{24} \mathrm{~N}_{4} \mathrm{O}_{2}+\mathrm{H}^{+}\right)$317.1972, found 317.1970.

\subsubsection{6. $N$-(tert-Butoxycarbonyl)-N-methyl-4-[(1-methyl-1H-1,2,3-triazol-4- yl)methyl] benzylamine 77}

It was prepared as described for 17. From compound 74 (140 mg, $0.46 \mathrm{mmol}), \mathrm{NaH}$ (60\% supension in mineral oil, $28 \mathrm{mg}, 0.70 \mathrm{mmol})$, and iodomethane $(0.03 \mathrm{~mL}, 68 \mathrm{mg}$, $0.48 \mathrm{mmol})$, stirring at $60{ }^{\circ} \mathrm{C}$ for $2 \mathrm{~h}$, treating again with $\mathrm{NaH}(60 \%$ supension in mineral oil, $28 \mathrm{mg}, 0.70 \mathrm{mmol})$, and iodomethane $(0.03 \mathrm{~mL}, 68 \mathrm{mg}, 0.48 \mathrm{mmol})$ and stirring at $60{ }^{\circ} \mathrm{C}$ for an additional $2 \mathrm{~h}$, compound $77(120 \mathrm{mg}, 83 \%$ yield) was obtained as a brown oil; $R_{f} 0.56\left(\mathrm{CH}_{2} \mathrm{Cl}_{2} / \mathrm{MeOH} / 50 \%\right.$ aq. $\mathrm{NH}_{4} \mathrm{OH}$ 9.5:0.5:0.05); IR (ATR) $v$ $1686(\mathrm{C}=\mathrm{O}) \mathrm{cm}^{-1} ;{ }^{1} \mathrm{H} \mathrm{NMR}\left(400 \mathrm{MHz}, \mathrm{CDCl}_{3}\right) \delta 1.46\left[\mathrm{br} \mathrm{s}, 9 \mathrm{H}, \mathrm{C}\left(\mathrm{CH}_{3}\right)_{3}\right], 2.79(\mathrm{br} \mathrm{s}$, $\left.3 \mathrm{H}, \mathrm{N}-\mathrm{CH}_{3}\right), 4.01$ (s, 3H, 1'- $\left.\mathrm{CH}_{3}\right), 4.05\left(\mathrm{~s}, 2 \mathrm{H}, 4-\mathrm{CH}_{2}\right), 4.37$ (br s, $\left.2 \mathrm{H}, 1-\mathrm{CH}_{2}-\mathrm{N}\right)$, 
$7.14(\mathrm{~d}, J=8.0 \mathrm{~Hz}, 2 \mathrm{H}), 7.21$ (d, $J=8.0 \mathrm{~Hz}, 2 \mathrm{H})$ (phenylene $\mathrm{CH}), 7.20$ (s, 1H, 5'-H); ${ }^{13} \mathrm{C}$ NMR $\left(100.6 \mathrm{MHz}, \mathrm{CDCl}_{3}\right) \delta 28.6\left[3 \mathrm{CH}_{3}, \mathrm{C}\left(\mathrm{CH}_{3}\right)_{3}\right], 32.0\left(\mathrm{CH}_{2}, 4-\mathrm{CH}_{2}\right), 34.0\left(\mathrm{CH}_{3}\right.$, $\left.\mathrm{N}-\mathrm{CH}_{3}\right), 36.7\left(\mathrm{CH}_{3}, 1^{\prime}-\mathrm{CH}_{3}\right), 51.7\left(\right.$ br $\left.\mathrm{CH}_{2}\right), 52.4\left(\right.$ br $\left.\mathrm{CH}_{2}\right)\left(1-\mathrm{CH}_{2}-\mathrm{N}\right), 79.8[\mathrm{C}$, $\left.C\left(\mathrm{CH}_{3}\right)_{3}\right], 122.5\left(\mathrm{CH}, \mathrm{C}^{\prime}\right.$ ), 127.8 (br CH), 128.0 (br CH), $128.9(\mathrm{CH}), 129.0(\mathrm{CH})$ (phenylene CH), 136.4 (C), 138.2 (C) (C1, C4), 148.0 (C, C4'), 155.9 (br C, NCOO); HRMS (ESI), calcd for $\left(\mathrm{C}_{17} \mathrm{H}_{24} \mathrm{~N}_{4} \mathrm{O}_{2}+\mathrm{H}^{+}\right) 317.1972$, found 317.1965 .

\subsubsection{N-Methyl-3-[(1-methyl-1H-1,2,3-triazol-4-yl)methyl]benzylamine 79}

It was prepared as described for 25 . From $76(384 \mathrm{mg}, 1.21 \mathrm{mmol})$ and $\mathrm{H}_{3} \mathrm{PO}_{4}(85 \%$ purity, $2.15 \mathrm{~mL}, 18.6 \mathrm{mmol}$ ), amine 79 (242 $\mathrm{mg}$, quantitative yield) was obtained as an orange oil; $R_{f} 0.30\left(\mathrm{CH}_{2} \mathrm{Cl}_{2} / \mathrm{MeOH} / 50 \%\right.$ aq. $\mathrm{NH}_{4} \mathrm{OH}$ 9.5:0.5:0.05).

79. $\mathrm{HCl}$ : sticky solid, IR (ATR) v 3400-2400 (max at 3374, 3116, 3074, 2956, 2778, 2699, 2542, 2417, ${ }^{+} \mathrm{N}-\mathrm{H}$ and $\left.\mathrm{C}-\mathrm{H}\right) \mathrm{cm}^{-1} ;{ }^{1} \mathrm{H}$ NMR (400 MHz, CD $\left.3 \mathrm{OD}\right) \delta 2.73(\mathrm{~s}, 3 \mathrm{H}$, $\left.\mathrm{N}-\mathrm{CH}_{3}\right), 4.21\left(\mathrm{~s}, 2 \mathrm{H}, 3-\mathrm{CH}_{2}\right), 4.32\left(\mathrm{~s}, 3 \mathrm{H}, 1{ }^{\prime}-\mathrm{CH}_{3}\right), 4.33\left(\mathrm{~s}, 2 \mathrm{H}, 1-\mathrm{CH}_{2}-\mathrm{N}\right), 4.99(\mathrm{~s}$, ${ }^{+} \mathrm{NH}_{2}$ ), 7.42-7.52 (complex signal, 3H, 4-H, 5-H, 6-H), 7.55 (br s, 1H, 2-H), 8.47 (s, $\left.1 \mathrm{H}, 5^{\prime}-\mathrm{H}\right) ;{ }^{13} \mathrm{C} \mathrm{NMR}\left(100.6 \mathrm{MHz}, \mathrm{CD}_{3} \mathrm{OD}\right) \delta 30.0\left(\mathrm{CH}_{2}, 3-\mathrm{CH}_{2}\right), 33.2\left(\mathrm{CH}_{3}, \mathrm{~N}-\mathrm{CH}_{3}\right)$, $40.0\left(\mathrm{CH}_{3}, 1{ }^{\prime}-\mathrm{CH}_{3}\right), 53.3\left(\mathrm{CH}_{2}, 1-\mathrm{CH}_{2}-\mathrm{N}\right), 129.5(\mathrm{CH}), 130.3(\mathrm{CH}), 131.1(\mathrm{CH}), 131.2$ $(\mathrm{CH}), 131.5(\mathrm{CH})$ (phenylene $\mathrm{CH}, \mathrm{C5}$ '), 133.6 (C, C1), 138.0 (C, C3), 144.5 (C, C4'); HRMS (ESI), calcd for $\left(\mathrm{C}_{12} \mathrm{H}_{16} \mathrm{~N}_{4}+\mathrm{H}^{+}\right)$: 217.1448, found 217.1446.

\subsubsection{N-Methyl-4-[(1-methyl-1H-1,2,3-triazol-4-yl)methyl]benzylamine 80}

It was prepared as described for 25 . From $77(237 \mathrm{mg}, 0.75 \mathrm{mmol})$ and $\mathrm{H}_{3} \mathrm{PO}_{4}(85 \%$ purity, $1.29 \mathrm{~mL}, 11.2 \mathrm{mmol}$ ), amine 80 (156 mg, 96\% yield) was obtained as an orange oil; $R_{f} 0.24\left(\mathrm{CH}_{2} \mathrm{Cl}_{2} / \mathrm{MeOH} / 50 \%\right.$ aq. $\mathrm{NH}_{4} \mathrm{OH}$ 9.5:0.5:0.05).

80 $\cdot \mathrm{HCl}$ : sticky solid, IR (ATR) v 3400-2400 (max at 3339, 3085, 2931, 2744, 2708, 2547, 2415, ${ }^{+} \mathrm{N}-\mathrm{H}$ and $\left.\mathrm{C}-\mathrm{H}\right) \mathrm{cm}^{-1} ;{ }^{1} \mathrm{H}$ NMR (400 MHz, CD $\mathrm{CD}_{3} \mathrm{O} \delta 2.71(\mathrm{~s}, 3 \mathrm{H}$, $\left.\mathrm{N}-\mathrm{CH}_{3}\right), 4.19\left(\mathrm{~s}, 2 \mathrm{H}, 4-\mathrm{CH}_{2}\right), 4.23\left(\mathrm{~s}, 3 \mathrm{H}, 1^{\prime}-\mathrm{CH}_{3}\right), 4.24\left(\mathrm{~s}, 2 \mathrm{H}, 1-\mathrm{CH}_{2}-\mathrm{N}\right), 4.90(\mathrm{~s}$, $\left.{ }^{+} \mathrm{NH}_{2}\right), 7.42(\mathrm{~d}, J=8.0 \mathrm{~Hz}, 2 \mathrm{H}), 7.51(\mathrm{~d}, J=8.0 \mathrm{~Hz}, 2 \mathrm{H})$ (phenylene $\left.\mathrm{CH}\right), 8.20(\mathrm{~s}, 1 \mathrm{H}$, 5 '-H); ${ }^{13} \mathrm{C}$ NMR (100.6 MHz, CD 3 OD) $\delta 30.6\left(\mathrm{CH}_{2}, 4-\mathrm{CH}_{2}\right), 33.1\left(\mathrm{CH}_{3}, \mathrm{~N}-\mathrm{CH}_{3}\right), 39.0$ $\left(\mathrm{CH}_{3}, 1^{\prime}-\mathrm{CH}_{3}\right), 53.1\left(\mathrm{CH}_{2}, 1-\mathrm{CH}_{2}-\mathrm{N}\right), 127.9(\mathrm{CH}, \mathrm{C} 5$ ') $130.7(2 \mathrm{CH}), 131.62(2 \mathrm{CH})$ (phenylene CH), 131.56 (C, C1), 139.7 (C, C4), 145.6 (C, C4'); HRMS (ESI), calcd for $\left(\mathrm{C}_{12} \mathrm{H}_{16} \mathrm{~N}_{4}+\mathrm{H}^{+}\right)$217.1448, found 217.1448. 


\subsubsection{N-Methyl-4-[(1-methyl-1H-1,2,3-triazol-4-yl)methyl]phenethylamine $\mathbf{8 1}$}

To a suspension of $\mathrm{LiAlH}_{4}(118 \mathrm{mg}, 3.11 \mathrm{mmol})$ in dry THF $(4 \mathrm{~mL})$, a solution of 75 $(165 \mathrm{mg}, 0.52 \mathrm{mmol})$ in dry THF $(4 \mathrm{~mL})$ was added dropwise at $0{ }^{\circ} \mathrm{C}$. The reaction mixture was stirred at reflux for $72 \mathrm{~h}$, cooled to $0{ }^{\circ} \mathrm{C}$, diluted with $1 \mathrm{~N} \mathrm{NaOH}(20 \mathrm{~mL})$, and extracted with $\mathrm{CH}_{2} \mathrm{Cl}_{2}(3 \times 15 \mathrm{~mL})$. The combined organic extracts were dried over anhydrous $\mathrm{Na}_{2} \mathrm{SO}_{4}$, filtered, and concentrated under reduced pressure to afford an oily residue (169 mg), which was purified through column chromatography (40-60 $\mu \mathrm{m}$ silica gel, $\mathrm{CH}_{2} \mathrm{Cl}_{2} / \mathrm{MeOH} / 50 \%$ aq. $\mathrm{NH}_{4} \mathrm{OH}$ mixtures, gradient elution). On elution with $\mathrm{CH}_{2} \mathrm{Cl}_{2} / \mathrm{MeOH} / 50 \%$ aq. $\mathrm{NH}_{4} \mathrm{OH}$ 95:5:0.2, amine 81 (53 mg, 44\% yield) was isolated as a yellow oil; $R_{f} 0.16\left(\mathrm{CH}_{2} \mathrm{Cl}_{2} / \mathrm{MeOH} / 50 \%\right.$ aq. $\mathrm{NH}_{4} \mathrm{OH}$ 9:1:0.05).

81· $\mathrm{HCl}$ : yellowish solid, mp $149-151^{\circ} \mathrm{C}$; IR (ATR) v 3400-2400 (max at 3395, 3118, 2938, 2771, 2450, ${ }^{+} \mathrm{N}-\mathrm{H}$ and $\left.\mathrm{C}-\mathrm{H}\right) \mathrm{cm}^{-1} ;{ }^{1} \mathrm{H}$ NMR (400 MHz, CD $\left.\mathrm{OD}\right) \delta 2.71(\mathrm{~s}, 3 \mathrm{H}$, $\left.\mathrm{N}-\mathrm{CH}_{3}\right), 2.97\left(\mathrm{t}, J=7.2 \mathrm{~Hz}, 2 \mathrm{H}, 1-\mathrm{CH}_{2}-\mathrm{CH}_{2}-\mathrm{N}\right), 3.23(\mathrm{t}, J=7.2 \mathrm{~Hz}, 2 \mathrm{H}$, $\left.1-\mathrm{CH}_{2}-\mathrm{CH}_{2}-\mathrm{N}\right), 4.06\left(\mathrm{~s}, 2 \mathrm{H}, 4-\mathrm{CH}_{2}\right), 4.09\left(\mathrm{~s}, 3 \mathrm{H}, 1{ }^{\prime}-\mathrm{CH}_{3}\right), 4.86\left(\mathrm{~s},{ }^{+} \mathrm{NH}_{2}\right), 7.24(\mathrm{dm}, J$ $=8.8 \mathrm{~Hz}, 2 \mathrm{H}), 7.27(\mathrm{dm}, J=8.8 \mathrm{~Hz}, 2 \mathrm{H})(\mathrm{Ph}-\mathrm{CH}), 7.81\left(\mathrm{~s}, 1 \mathrm{H}, 5{ }^{\prime}-\mathrm{H}\right) ;{ }^{13} \mathrm{C}$ NMR $(100.6$ $\left.\mathrm{MHz}, \mathrm{CD}_{3} \mathrm{OD}\right) \delta 31.7\left(\mathrm{CH}_{2}, 4-\mathrm{CH}_{2}\right), 32.9\left(\mathrm{CH}_{2}, 1-\mathrm{CH}_{2}-\mathrm{CH}_{2}-\mathrm{N}\right), 33.7\left(\mathrm{CH}_{3}, \mathrm{~N}-\mathrm{CH}_{3}\right)$, $37.5\left(\mathrm{CH}_{3}, 1^{\prime}-\mathrm{CH}_{3}\right), 51.4\left(\mathrm{CH}_{2}, 1-\mathrm{CH}_{2}-\mathrm{CH}_{2}-\mathrm{N}\right), 125.5(\mathrm{CH}, \mathrm{C} 5$ '), $130.1(2 \mathrm{CH}), 130.4$ (2CH) (Ph-CH), 136.0 (C), 139.0 (C) (Ph-C1, Ph-C4), 147.7 (C, C4'); HRMS (ESI), calcd for $\left(\mathrm{C}_{13} \mathrm{H}_{18} \mathrm{~N}_{4}+\mathrm{H}+\right)$ 231.1604, found 231.1605.

\subsubsection{N-Methyl-N-\{3-[(1-methyl-1H-1,2,3-triazol-4-yl)methyl]benzyl\}-N- propargylamine $\mathbf{8 2}$}

It was prepared as described for 31. From amine 79 (236 mg, $1.09 \mathrm{mmol}), \mathrm{Cs}_{2} \mathrm{CO}_{3}(354$ $\mathrm{mg}, 1.09 \mathrm{mmol})$, and propargyl bromide $(80 \%$ solution in toluene, $0.16 \mathrm{~mL}, 1.08$ mmol), and stirring at at $0{ }^{\circ} \mathrm{C}$ for $30 \mathrm{~min}$ and at $\mathrm{rt}$ for an additional $3 \mathrm{~h}$, propargylamine 82 (180 mg, 66\% yield) was obtained as a yellow oil, without the need of chromatographic purification; $R_{f} 0.66\left(\mathrm{CH}_{2} \mathrm{Cl}_{2} / \mathrm{MeOH} / 50 \%\right.$ aq. $\mathrm{NH}_{4} \mathrm{OH}$ 9.5:0.5:0.05). 82. $\mathrm{HCl}$ : sticky solid; IR (ATR) v 3400-2400 (max at 3389, 3191, 3023, 2930, 2491, ${ }^{+} \mathrm{N}-\mathrm{H}, \equiv \mathrm{C}-\mathrm{H}$, and $\left.\mathrm{C}-\mathrm{H}\right), 2122(\mathrm{C} \equiv \mathrm{C}) \mathrm{cm}^{-1} ;{ }^{1} \mathrm{H}$ NMR (400 MHz, CD $\left.{ }_{3} \mathrm{OD}\right) \delta 2.94(\mathrm{~s}$, $3 \mathrm{H}, \mathrm{N}-\mathrm{CH}_{3}$ ), 3.47 (t, $J=2.8 \mathrm{~Hz}, 1 \mathrm{H}$, propargyl $\mathrm{CH}$ ), 4.08 (br s, $2 \mathrm{H}$, propargyl $\mathrm{CH}_{2}$ ), $4.26\left(\mathrm{~s}, 3 \mathrm{H}, 1\right.$ ' $\left.-\mathrm{CH}_{3}\right), 4.30\left(\mathrm{~s}, 2 \mathrm{H}, 3-\mathrm{CH}_{2}\right), 4.47$ (br signal, $\left.2 \mathrm{H}, 1-\mathrm{CH}_{2}-\mathrm{N}\right), 5.04$ (s, ${ }^{+} \mathrm{NH}$ ), 7.48-7.56 (complex signal, 3H, 4-H, 5-H, 6-H), 7.61 (br s, 1H, 2-H), 8.33 (s, 1H, 5 ' $-H) ;{ }^{13} \mathrm{C}$ NMR (100.6 MHz, $\left.\mathrm{CD}_{3} \mathrm{OD}\right) \delta 30.5\left(\mathrm{CH}_{2}, 3-\mathrm{CH}_{2}\right), 39.3\left(\mathrm{CH}_{3}, 1\right.$ ' $\left.-\mathrm{CH}_{3}\right), 40.1$ 
$\left(\mathrm{CH}_{3}, \mathrm{~N}-\mathrm{CH}_{3}\right), 45.4\left(\mathrm{CH}_{2}\right.$, propargyl $\left.\mathrm{CH}_{2}\right), 59.4\left(\mathrm{CH}_{2}, 1-\mathrm{CH}_{2}-\mathrm{N}\right), 72.8(\mathrm{C}$, propargyl C), $81.9(\mathrm{CH}$, propargyl $\mathrm{CH}), 128.4\left(\mathrm{CH}, \mathrm{C}^{\prime}\right), 131.0(\mathrm{CH}), 131.2(\mathrm{CH}+\mathrm{C}), 131.8$ $(\mathrm{CH}), 132.6(\mathrm{CH})(\mathrm{C} 1$, phenylene $\mathrm{CH}), 139.2$ (C, C3), 145.2 (C, C4'); HRMS (ESI), calcd for $\left(\mathrm{C}_{15} \mathrm{H}_{18} \mathrm{~N}_{4}+\mathrm{H}^{+}\right) 255.1604$, found 255.1600 .

\subsubsection{N-Methyl-N-\{4-[(1-methyl-1H-1,2,3-triazol-4-yl)methyl]benzyl\}-N-}

\section{propargylamine $\mathbf{8 3}$}

It was prepared as described for 31. From amine 80 (131 mg, $0.61 \mathrm{mmol}), \mathrm{Cs}_{2} \mathrm{CO}_{3}(0.20$ $\mathrm{g}, 0.61 \mathrm{mmol}$ ), and propargyl bromide ( $80 \%$ solution in toluene, $0.07 \mathrm{~mL}, 0.47 \mathrm{mmol}$ ), and stirring at at $0{ }^{\circ} \mathrm{C}$ for $3.5 \mathrm{~h}$, an orange oily residue (122 $\left.\mathrm{mg}\right)$ was obtained and purified through column chromatography $\left(40-60 \mu \mathrm{m}\right.$ silica gel, $\mathrm{CH}_{2} \mathrm{Cl}_{2} / 50 \%$ aq. $\mathrm{NH}_{4} \mathrm{OH}$ 100:0.2), to afford propargylamine 83 (48 mg, 40\% yield) as a yellow oil; $R_{f}$ $0.67\left(\mathrm{CH}_{2} \mathrm{Cl}_{2} / \mathrm{MeOH} / 50 \%\right.$ aq. $\mathrm{NH}_{4} \mathrm{OH}$ 9:1:0.05).

83. $\mathrm{HCl}: \mathrm{mp} 156-158{ }^{\circ} \mathrm{C}$; IR (ATR) v 3400-2400 (max at 3178, 3095, 2923, 2692, 2501, 2418, ${ }^{+} \mathrm{N}-\mathrm{H}, \equiv \mathrm{C}-\mathrm{H}$, and $\left.\mathrm{C}-\mathrm{H}\right), 2120(\mathrm{C} \equiv \mathrm{C}) \mathrm{cm}^{-1} ;{ }^{1} \mathrm{H}$ NMR $\left(400 \mathrm{MHz}, \mathrm{CD}_{3} \mathrm{OD}\right)$ $\delta 2.92\left(\mathrm{~s}, 3 \mathrm{H}, \mathrm{N}-\mathrm{CH}_{3}\right), 3.47(\mathrm{t}, J=2.8 \mathrm{~Hz}, 1 \mathrm{H}$, propargyl $\mathrm{CH}), 4.05$ (br signal, $2 \mathrm{H}$, propargyl $\left.\mathrm{CH}_{2}\right), 4.19\left(\mathrm{~s}, 3 \mathrm{H}, 1^{\prime}-\mathrm{CH}_{3}\right), 4.22\left(\mathrm{~s}, 2 \mathrm{H}, 4-\mathrm{CH}_{2}\right), 4.41$ (br signal, $2 \mathrm{H}$, $\left.1-\mathrm{CH}_{2}-\mathrm{N}\right), 4.86\left(\mathrm{~s},{ }^{+} \mathrm{NH}\right), 7.44(\mathrm{~d}, J=8.4 \mathrm{~Hz}, 2 \mathrm{H}), 7.52(\mathrm{~d}, J=8.4 \mathrm{~Hz}, 2 \mathrm{H})$ (phenylene $\mathrm{CH}), 8.10\left(\mathrm{~s}, 1 \mathrm{H}, 5^{\prime}-\mathrm{H}\right) ;{ }^{13} \mathrm{C} \mathrm{NMR}\left(100.6 \mathrm{MHz}, \mathrm{CD}_{3} \mathrm{OD}\right) \delta 30.6\left(\mathrm{CH}_{2}, 4-\mathrm{CH}_{2}\right), 39.0$ $\left(\mathrm{CH}_{3}, 1^{\prime}-\mathrm{CH}_{3}\right), 40.1\left(\mathrm{CH}_{3}, \mathrm{~N}-\mathrm{CH}_{3}\right), 45.4\left(\mathrm{CH}_{2}\right.$, propargyl $\left.\mathrm{CH}_{2}\right), 59.3\left(\mathrm{CH}_{2}, 1-\mathrm{CH}_{2}-\mathrm{N}\right)$, 72.8 (C, propargyl C), 81.9 (CH, propargyl CH), 128.0 (CH, C5'), $129.4(\mathrm{C}, \mathrm{C} 1), 130.9$ (2CH), $132.9(2 \mathrm{CH})$ (phenylene $\mathrm{CH}), 140.5$ (C, C4), 145.5 (C, C4'); HRMS (ESI), calcd for $\left(\mathrm{C}_{15} \mathrm{H}_{18} \mathrm{~N}_{4}+\mathrm{H}^{+}\right) 255.1604$, found 255.1599.

4.1.72. N-methyl-N-\{4-[(1-methyl-1H-1,2,3-triazol-4-yl)methyl]phenethyl $\}-N$ propargylamine 84

It was prepared as described for 31. From amine 81 (30 mg, $0.13 \mathrm{mmol}), \mathrm{Cs}_{2} \mathrm{CO}_{3}(42$ $\mathrm{mg}, 0.13 \mathrm{mmol})$, and propargyl bromide $(80 \%$ solution in toluene, $0.02 \mathrm{~mL}, 0.13$ $\mathrm{mmol}$ ), and stirring at $0{ }^{\circ} \mathrm{C}$ for $30 \mathrm{~min}$ and at $\mathrm{rt}$ for an additional $3 \mathrm{~h}$, an oily residue (48 $\mathrm{mg}$ ) was obtained and purified through column chromatography $(40-60 \mu \mathrm{m}$ silica gel, $\mathrm{CH}_{2} \mathrm{Cl}_{2}$ ), to afford propargylamine 84 (20 mg, 57\% yield) as a yellow oil; $R_{f} 0.55$ $\left(\mathrm{CH}_{2} \mathrm{Cl}_{2} / \mathrm{MeOH} / 50 \%\right.$ aq. $\mathrm{NH}_{4} \mathrm{OH}$ 9:1:0.05). 
84·HCl: sticky solid; IR (ATR) v 3500-2400 (max at 3413, 3358, 3213, 3068, 2944, 2496, ${ }^{+} \mathrm{N}-\mathrm{H}, \equiv \mathrm{C}-\mathrm{H}$, and $\left.\mathrm{C}-\mathrm{H}\right), 2144(\mathrm{C} \equiv \mathrm{C}) \mathrm{cm}^{-1} ;{ }^{1} \mathrm{H}$ NMR (400 MHz, CD $\left.\mathrm{OD}\right) \delta 3.02$ (s, $\left.3 \mathrm{H}, \mathrm{N}-\mathrm{CH}_{3}\right), 3.06\left(\mathrm{t}, J=6.8 \mathrm{~Hz}, 2 \mathrm{H}, 1-\mathrm{CH}_{2}-\mathrm{CH}_{2}-\mathrm{N}\right), 3.41(\mathrm{~m}, 1 \mathrm{H}$, propargyl $\mathrm{CH})$, 3.48 (br signal, 2H, 1- $\left.\mathrm{CH}_{2}-\mathrm{CH}_{2}-\mathrm{N}\right), 4.09\left(\mathrm{~s}, 2 \mathrm{H}\right.$, propargyl $\left.\mathrm{CH}_{2}\right), 4.12(\mathrm{~s}, 3 \mathrm{H}$, 1 ' $\left.-\mathrm{CH}_{3}\right), 4.18\left(\mathrm{~s}, 2 \mathrm{H}, 4-\mathrm{CH}_{2}\right), 4.86\left(\mathrm{~s},{ }^{+} \mathrm{NH}\right), 7.28$ (complex signal, $\left.4 \mathrm{H}, \mathrm{Ph}-\mathrm{CH}\right), 7.89$ (s, 1H, 5'-H); ${ }^{13} \mathrm{C}$ NMR (100.6 MHz, CD $\left.3 \mathrm{OD}\right) \delta 31.1\left(\mathrm{CH}_{2}\right), 31.4\left(\mathrm{CH}_{2}\right)\left(4-\mathrm{CH}_{2}\right.$, 1- $\left.\mathrm{CH}_{2}-\mathrm{CH}_{2}-\mathrm{N}\right), 37.9\left(\mathrm{CH}_{3}, 1\right.$ ' $\left.-\mathrm{CH}_{3}\right), 40.7\left(\mathrm{CH}_{3}, \mathrm{~N}-\mathrm{CH}_{3}\right), 46.3\left(\mathrm{CH}_{2}\right.$, propargyl $\left.\mathrm{CH}_{2}\right)$, $57.5\left(\mathrm{CH}_{2}, 1-\mathrm{CH}_{2}-\mathrm{CH}_{2}-\mathrm{N}\right), 72.8(\mathrm{C}$, propargyl $\mathrm{C}), 81.5(\mathrm{CH}$, propargyl $\mathrm{CH}), 126.1$ (CH, C5'), 130.3 (2CH), 130.4 (2CH) (Ph-CH), 135.7 (C), 138.7 (C) (Ph-C1, Ph-C4), 147.3 (C, C4'); HRMS (ESI), calcd for $\left(\mathrm{C}_{16} \mathrm{H}_{20} \mathrm{~N}_{4}+\mathrm{H}^{+}\right) 269.1761$, found 269.1760 .

\subsection{Biological assays}

\subsubsection{Inhibition of hrMAO-A and hrMAO-B}

The activity of hrMAO-A and hrMAO-B (Sigma-Aldrich, Madrid, Spain) was determined using Amplex UltraRed fluorometric coupled method. For both MAO isoforms, tyramine hydrochloride was used as substrate and enzymatic assays were performed in 96-well black opaque microplates (OptiPlate-96F, PerkinElmer) in a final volume of $200 \mu \mathrm{L}$. Serial dilutions of each inhibitor were pre-incubated with either 0.36 $\mathrm{U} / \mathrm{mL} h r \mathrm{MAO}$ A or $0.0675 \mathrm{U} / \mathrm{mL} h r \mathrm{MAO} \mathrm{B}$ for $30 \mathrm{~min}$ at $37^{\circ} \mathrm{C}$. Enzymatic reactions were started upon the addition of $100 \mu \mathrm{L}$ of a mixture solution containing $1 \mathrm{mM}$ tyramine, $0.04 \mathrm{U} / \mathrm{mL}$ horseradish peroxidase (HRP) and $25 \mathrm{mM}$ Amplex UltraRed ${ }^{\circledR}$ reagent in $0.25 \mathrm{mM}$ sodium phosphate $(\mathrm{pH} 7.4)$ as final concentrations. The fluorescence production of resorufin was measured for at least $30 \mathrm{~min}$ at 530/590 $\mathrm{nm}$ in a spectrophotometric plate reader (FluoStar OPTIMA, BM G Labtech). The enzymatic activity in the absence of compound was used to determine $100 \%$ enzyme activity. The potential capacity of the compounds to interfere with the fluorescence generated in the reaction was determined by adding the compounds to solutions in the absence of MAO. From dose-response curves, $\mathrm{IC}_{50}$ values were accordingly calculated using the GraphPad 'PRISM' software (version 5.0). Data are expressed as mean \pm SEM of at least three different experiments performed in duplicate. Clorgyline and $R$-deprenyl (Sigma-Aldrich) were used as reference compounds.

\subsubsection{Reversibility of hrMAO-B inhibition}


To study the reversibility of the inhibition of hrMAO-B by compound 33, 100-fold enzyme concentration was inhibited by $200 \mathrm{nM} L$-deprenyl or $50 \mathrm{nM} 33$ (10-fold $\mathrm{IC}_{50}$ values) for $1 \mathrm{~h}$ at $37^{\circ} \mathrm{C}$. Following pre-incubation times, enzyme solution was rapidly diluted (100-fold) in a mix solution containing $1 \mathrm{mM} p$-tyramine, HRP and Amplex UltraRed $^{\circledR}$ reagent in $50 \mathrm{mM}$ phosphate buffer $\mathrm{pH}$ 7.4. Next, $h r$ MAO-B activity was followed at $530 / 590 \mathrm{~nm}$ for $40 \mathrm{~min}$.

\subsubsection{Time-dependent inhibition of hrMAO-B}

To investigate the time-dependent inhibition of $h r \mathrm{MAO}-\mathrm{B}$ activity by compound $\mathbf{3 3}$, the enzyme was inhibited by $10 \mu \mathrm{M} 33$ for at different pre-incubation times (0-360 min). At the end of each pre-incubation, activity was measured as previously described and plotted as percentage of control samples versus pre-incubation time.

\subsubsection{Determination of brain permeability: PAMPA-BBB assay}

The in vitro permeability $\left(P_{\mathrm{e}}\right)$ of the novel 1,2,3-triazole-based compounds and fourteen known drugs (Table 2) through lipid extract of porcine brain membrane was determined by using a parallel artificial membrane permeation assay ${ }^{82}$ using a mixture PBS:EtOH 70:30. Assay validation was made by comparison of the experimental $P_{\mathrm{e}}$ values of the known drugs with their reported values, which showed a good correlation: $P e(\exp )=$ $1.003 P e($ lit $)-0.783\left(R^{2}=0.93\right)$. From this equation and the limits established by Di $e t$ al. for BBB permeation, ${ }^{82}$ three ranges of permeability were established: compounds of high BBB permeation (CNS+): $P e\left(10^{-6} \mathrm{~cm} \mathrm{~s}^{-1}\right)>5.18$; compounds of low BBB permeation (CNS-): $P e\left(10^{-6} \mathrm{~cm} \mathrm{~s}^{-1}\right)<2.06$; compounds of uncertain BBB permeation $(\mathrm{CNS} \pm)$ : and $5.18>P \mathrm{e}\left(10^{-6} \mathrm{~cm} \mathrm{~s}^{-1}\right)>2.06$.

Table 2. Reported and experimental permeability values $\left(P_{\mathrm{e}} 10^{-6} \mathrm{~cm} \mathrm{~s}^{-1}\right)$ of 14 commercial drugs used for the PAMPA-BBB assay validation

\begin{tabular}{|c|c|c|}
\hline Compound & $\begin{array}{c}\text { Literature } \\
\text { value }^{\mathrm{a}}\end{array}$ & $\begin{array}{c}\text { Experimental } \\
\text { value }^{\mathrm{b}}\end{array}$ \\
\hline
\end{tabular}




\begin{tabular}{lll}
\hline Cimetidine & 0.0 & $0.70 \pm 0.03$ \\
Lomefloxacin & 0.0 & $0.78 \pm 0.04$ \\
Norfloxacin & 0.1 & $0.90 \pm 0.02$ \\
Ofloxacin & 0.8 & $0.98 \pm 0.06$ \\
Hydrocortisone & 1.9 & $1.40 \pm 0.05$ \\
Piroxicam & 2.5 & $1.93 \pm 0.11$ \\
Clonidine & 5.3 & $6.50 \pm 0.05$ \\
Corticosterone & 5.1 & $6.70 \pm 0.10$ \\
Imipramine & 13 & $12.3 \pm 0.1$ \\
Promazine & 8.8 & $13.8 \pm 0.3$ \\
Progesterone & 9.3 & $16.8 \pm 0.1$ \\
Desipramine & 12 & $17.8 \pm 0.1$ \\
Testosterone & 17 & $24.0 \pm 1.4$ \\
Verapamil & 16 & $28.1 \pm 1.6$ \\
\hline
\end{tabular}

${ }^{a}$ Taken from ref. [82].

${ }^{\mathrm{b}}$ Values are expressed as the mean $\pm \mathrm{SD}$ of three independent experiments.

\subsection{Docking studies}

The binding mode of selected 1,2,3-triazole derivatives was explored by means of docking calculations carried out with Glide, using an empirical scoring function calibrated on the basis of protein-ligand complexes. ${ }^{84}$ Docking was performed using the MAO-B X-ray structure of the complex with inhibitor 6 (PDB entry 4CRT) ${ }^{80}$ and deprenyl (PDB entry 2BYB), ${ }^{28}$ but no significant differences were found for the best ligand poses. The protocol defined in our previous studies was adopted here. ${ }^{39}$ Briefly, the docking volume was defined as the space covered by the binding cavity in MAO-B. Suitable restraints were introduced to keep the amine nitrogen of the proargylamine unit close to the position found for the corresponding atom in deprenyl and rasagiline (PDB entry $2 \mathrm{BK} 4)^{79}$ in order to mimic the orientation after irreversible linkage with the FAD. Each compound was subjected to 100 docking runs. Whereas the protein was kept rigid, Glide accounts for the conformational flexibility of the ligand around rotatable bonds during docking calculations. The output docking modes were analyzed by visual inspection in conjunction with the docking scores.

\section{Acknowledgements}


We thank the financial support from Ministerio de Economía y Competitividad (SAF2014-57094-R) and the Generalitat de Catalunya (GC; 2014SGR52 and 2014SGR1189). We are grateful to the Consorci de Serveis Universitaris de Catalunya for computational resources. FJL acknowledges the support from ICREA Academia. Fellowships from GC to O.D.P., N.A., E.V., J.J.-J. and I.S. are gratefully acknowledged. 


\section{References}

[1] Prince, M.; Wimo, A.; Guerchet, M.; Ali, G.-C.; Wu, Y.-T.; Prina, M. World Alzheimer Report 2015. The global impact of dementia. An analysis of prevalence, incidence, cost \& trends; Alzheimer's Disease International: London, 2015; http://www.alz.co.uk.

[2] Bolea, I.; Gella, A.; Unzeta, M. Propargylamine-derived mutitarget-directed ligands: fighting Alzheimer's disease with monoamine oxidase inhibitors. $J$. Neural Transm. 2013, 120, 893-902.

[3] Guzior, N.; Wieckowska, A.; Panek, D.; Malawska, B. Recent development of multifunctional agents as potential drug candidates for the treatment of Alzheimer's disease. Curr. Med. Chem. 2015, 22, 373-404.

[4] Perry, E.K.; Perry, R.H.; Blessed, G.; Tomlinson, B.E. Necropsy evidence of central cholinergic deficits in senile dementia. Lancet 1977, 1, 189-189.

[5] Coyle, J.T.; Puttfarcken, P. Oxidative stress, glutamate, and neurodegenerative disordes. Science 1993, 262, 689-695.

[6] Perry, G.; Raina, A.K.; Nunomura, A.; Wataya, T.; Sayre, L.M.; Smith, M.A. How important is oxidative damage? Lessons from Alzheimer's disease. Free Radic. Biol.Med. 2000, 28, 831-834.

[7] Gella, A.; Durany, N. Oxidative stress in Alzheimer's disease. Cell. Adh. Migr. 2009, 3, 88-93.

[8] Huang X.; Moir, R.D.; Tanzi, R.E.; Bush, A.I.; Rogers, J.T. Redox-active metals, oxidative stress, and Alzheimer's disease pathology. Ann. N. Y. Acad. Sci. 2004, $1012,153-163$.

[9] Mishizen-Eberz, A.J.; Rissman, R.A.; Carter, T.L.; Ikonomovic, M.D.; Wolfe, B.B.; Armstrong, D.M. Biochemical and molecular studies of NMDA receptor subunits NR1/2A/2B in hippocampal subregions throughout progression of Alzheimer's disease pathology. Neurobiol. Dis. 2004, 15, 80-92.

[10] Goedert, M.; Spillantini, M.G.; Jakes, R.; Rutherford, D.; Crowther, R.A. Multiple isoforms of human microtubule-associated protein tau: sequences and localization in neurofibrillary tangles of Alzheimer's disease. Neuron. 1989, 3, $519-526$.

[11] Glenner, G.G.; Murphy, M.A. Amyloidosis of the nervous system. J. Neurol. Sci. 1989, 94, 1-28. 
[12] Birks, J.; Harvey, R. Donepezil for dementia due to Alzheimer's disease. Cochrane Database Syst. Rev. 2006, 1, CD001190.

[13] Birks, J.; Grimley, E.J.; Iakovidou, V.; Tsolaki, M. Rivastigmine for Alzheimer's disease. Cochrane Database Syst. Rev. 2000, 4, CD001191.

[14] Loy, C.; Schneider, L. Galantamine for Alzheimer's disease. Cochrane Database Syst.Rev. 2004, 4, CD001747.

[15] Areosa, S.A.; McShane, R.; Sheriff, F. Memantine for dementia. Cochrane Database Syst.Rev. 2005, 4, CD003154.

[16] Chen, X.; Decker, M. Multi-target compounds acting in the central nervous system designed from natural products. Curr. Med. Chem. 2013, 20, 1673-1685.

[17] Galdeano, C.; Viayna, E.; Arroyo, P.; Bidon-Chanal, A.; Blas, J.R.; MuñozTorrero, D.; Luque, F.J. Structural determinants of the multifactorial profile of dual binding site acetylcholinesterase inhibitors as anti-Alzheimer agents. Curr. Pharm. Design 2010, 16, 2818-2836.

[18] Matinez, A. Emerging drugs and targets for Alzheimer's disease. Vol. 1. BetaAmyloid, tau protein and glucose metabolism. (Ed.) RSC, 2010.

[19] Thomas, T. Monoamine oxidase B inhibitors in the treatment of Alzheimer's disease. Neurobiol. Aging 2000, 21, 343-348.

[20] Riederer, P.; Danyelczyk, W.; Grunblat, E. Monoamine oxidase inhibition in Alzheimer's disease. Neurotoxicology 2004, 25, 271-277.

[21] Kristal, B.S.; Conway, A.D.; Brown, A.M.; Jain, J.C.; Ulluci, P.A.; Li, S.W.; Burke, W.J. Selective dopaminergic vulnerability: 3,4dihydroxyphenylacetaldehyde targets mitochondria. Free Radic. Biol. Med. 2001, 30, 924-931.

[22] Edmondson, D.E.; Binda, C.; Wang, J.; Upadhyay, A.K.; Mattevi, A. Molecular and mechanistic properties of the membrane-bound mitochondrial monoamine oxidases. Biochemistry 2009, 48, 4220-4230.

[23] Tripton, K.F.; Boyce, S.; O’Sullivan, J.; Davey, G.P.; Healy, J. Monoamine oxidases: certainties and uncertainties. Curr. Med. Chem. 2004, 11, 1965-1982.

[24] Chen, K.; Holschneider, D.P.; Wu, W.; Rebrin, I.; Shih, J.C. A spontaneous point mutation produces monoamine oxidase A/B knock-out mice with greatly elevated monoamines and anxiety-like behavior. J. Biol. Chem. 2004, 279, 39645-39652. 
[25] Binda, C.; Newton-Vinson, P.; Hubálek, F.; Edmonson, D.E.; Mattevi, A. Structure of humane monoamine oxidase B, a drug target for the treatment of neurological disorders. Nat. Struct. Biol. 2002, 9, 22-26.

[26] Binda, C.; Li, M.; Hubálek, F.; Restelli, N.; Edmonson, D.E.; Mattevi, A. Insights into the mode of inhibition of human mitochondrial monoamine oxidase B from high-resolution crystal structures. Proc. Natl. Acad. Sci. U.S.A. 2003, 100, 9750-9755.

[27] Binda, C.; Hubálek, F.; Li, M.; Herzig, Y.; Sterling, J.; Edmonson, D.E.; Mattevi, A. Crystal structures of monoamine oxidase B in complex with four inhibitors of the $N$-propargylaminoindan class. J. Med. Chem. 2004, 47, 17671774 .

[28] De Colibus, L.; Li, M.; Binda, C.; Lustig, A.; Edmonson, D.E.; Mattevi, A. Three-dimensional structure of human monoamine oxidase A (MAO A): relation to the structure of rat MAO A and human MAO B. Proc. Natl. Acad. Sci. U.S.A. 2005, 102, 12684-12689.

[29] Son, S.Y.; Ma, J.; Kondou, Y.; Yoshimura, M.; Yamashita, E.; Tsukihara, T. Structure of human monoamine oxidase $\mathrm{A}$ at $2.2-\AA$ resolution: the control of opening the entry for substrates/inhibitors. Proc. Natl. Acad. Sci. U.S.A. 2008, 105, 5739-5744.

[30] Juárez-Jiménez, J.; Mendes, E.; Galdeano, C.; Martins, C.; Silva, D.B.; MarcoContelles, J.; do Carmo Carreiras, M.; Luque, F.J.; Ramsay, R.R. Exploring the structural basis of the selective inhibition of monoamine oxidase A by dicarbonitrile aminoheterocycles: role of Asn181 and Ile335 validated by spectroscopic and computational studies. Biochim. Biophys. Acta 2014, 1844, 389-397.

[31] Song, M.S.; Matveychuk, D.; MacKenzie, E.M.; Duchcherer, M.; Mousseau, D.D.; Baker, G.B. An update of monoamine oxidase inhibitors: multifaceted drugs. Prog. Neuropsychopharmacol. Biol. Psychiatry 2013, 44, 118-124.

[32] Burke, W.J.; Li, S.W.; Chung, H.D.; Ruggiero, D.A.; Kristal, B.S.; Johnson, E.M.; Lampe, P.; Kumar, V.B.; Franko, M.; Williams, E.A.; Zahm, D.S. Neurotoxicity of MAO metabolites of catecholamine neurotransmitters: role in neurodegenerative diseases. Neurotoxicology 2004, 25, 101-115.

[33] Naoi, M.; Maruyama, W.; Yi, H.; Akao, Y.; Yamaoka, Y.; Shamoto-Nagai, M. Neuroprotection by propargylamines in Parkinson's disease: intracellular 
mechanism underlying the anti-apoptotic function and search for clinical markers. J. Neural Transm. Suppl. 2007, 72, 121-131.

[34] Weinreb, O.; Amit, T.; Bar-Am, O.; Sagi, Y.; Mandel, S.; Youdim, M.B. (2006) Involvement of multiple survival signal transduction pathways in the neuroprotective, neurorescue and APP processing activity of rasagiline and its propargyl moiety. J. Neural Transm. Suppl. 2006, 70, 457-465.

[35] Yáñez, M.; Viña, D. Dual inhibitors of monoamine oxidase and cholinesterase for the treatment of Alzheimer disease. Curr. Top. Med. Chem. 2013, 13, 16921706 .

[36] Jo, S.; Yarishkin, O.; Hwang, Y.J.; Chun, Y.E.; Park, M.; Woo, D.H.; Bae, J.Y.; Kim, T.; Lee, J.; Chun, H.; Park, H.J.; Lee, D.Y.; Hong, J.; Kim, H.Y.; Oh, S.-J.; Park, S.J.; Lee, H.; Yoon, B.-E.; Kim, Y.S.; Jeong, Y.; Shim, I.; Bae, Y.C.; Cho, J.; Kowall, N.W.; Ryu, H.; Hwang, E.; Kim, D.; Lee, C.J. GABA from reactive astrocytes impairs memory in mouse models of Alzheimer's disease. Nat. Med. 2014, 20, 886-896.

[37] Weinreb, O.; Amit, T.; Bar-Am, O.; Youdim, M.B. Ladostigil: a novel multimodal neuroprotective drug with cholinesterase and brain-selective monoamine oxidase inhibitory activities for Alzheimer's disease treatment. Curr. Drug Targets 2012, 13, 483-494.

[38] Sterling, J.; Herzig, Y.; Goren, T.; Finkelstein, N.; Lerner, D.; Goldenberg, W.; Mikcolczi, I.; Molnar, S.; Rantal, F.; Tamas, T.; Toth, G.; Zagyva, A.; Zekany, A.; Finberg, J.; Lavian, G.; Gross, A.; Friedman, R.; Razin, M.; Huang, W.; Krais, B.; Chorev, M.; Youdim, M.B.; Weinstock, M. Novel dual inhibitors of $\mathrm{AChE}$ and MAO derived from hydroxy aminoindan and phenethylamine as potential treatment for Alzheimer's disease. J. Med. Chem. 2002, 54, 52605279 .

[39] Bolea, I.; Juárez-Jiménez, J.; de los Ríos, C.; Chioua, M.; Pouplana, R.; Luque, F.J.; Unzeta, M. Synthesis, biological evaluation and molecular modelling of donepezil and N-[(5-(benzyloxy)-1-methyl-1H-indol-2-yl) methyl]-Nmethylprop-2-yn-1-amine hybrids as new multipotent cholinesterase/monoamine oxidase inhibitors for the treatment of Alzheimer's disease. J. Med. Chem. 2011, $54,8251-8270$.

[40] Bolea, I.; Gella, A.; Monjas, I.; Perez, C.; Rodríguez-Franco, M. I.; MarcoContelles, J.; Samadi, A.; Unzeta, M. Multipotent, permeable drug ASS234 
inhibits $A \beta$ aggregation, possesses antioxidant properties and protects from $A \beta$ induced apoptpsis in vitro. Curr. Alzheimer Res. 2013, 9, 797-808.

[41] Farina, R.; Pisani, L.; Catto, M.; Nicolotti, O.; Gadaleta, D.; Denora, N.; SotoOtero, R.; Mendez-Alvarez, E.; Passos, C. S.; Muncipinto, G.; Altomare, C. D.; Nurisso, A.; Carrupt, P.-A.; Carotti, A. Structure-based design and optimization of multitarget-directed $2 \mathrm{H}$-chromen-2-one as potent inhibitors of monoamine oxidase B and cholinesterases. J. Med. Chem. 2015, 58, 5561-5578.

[42] Xie, S.S.; Wang, X.; Jiang, N.; Yu, W.; Wang, K.D.G.; Lan, J.S.; Li, Z.R.; Kong L.Y. Multi-target tacrine-coumarin hybrids: cholinesterase and monoamine oxidase B inhibition properties against Alzheimer's disease. Eur. J. Med. Chem. 2015, 95, 153-165.

[43] Zheng, H.; Youdim, M.B.H.; Fridkin, M. Site-activated multifunctional chelator with acetylcholinesterase and neuroprotective/neurorestorative moieties for Alzheimer's therapy. J. Med. Chem. 2009, 52, 4095-4098.

[44] Zheng, H.; Youdim, M.B.; Fridkin, M. Site-activated chelators targeting AChE and MAO for Alzheimer's therapy. ACS Chem. Biol. 2010, 5, 603-610.

[45] Passos, C.S.; Simões-Pires, C.A.; Nurisso, A.; Soldi, T.C.; Kato, L.; de Oliveira, C.M.; de Faria, E.O.; Marcourt, L.; Gottfried, C.; Carrupt, P.-A.; Henriques, A.T. Indole alkaloids of psychotria as multifunctional cholinesterases and monoamine oxidases inhibitors. Phytochemistry 2013, 86, 8-20.

[46] Pisani, L.; Farina, R.; Soto-Otero, R.; Denora, N.; Mangiatordi, G.; Nicolotti, O.; Mendez-Alvarez, E.; Cosimo, A.; Catto, M.; Carotti, A. Searching for multitarget neurotherapeutics against Alzheimer's: Discovery of potent AChE-MAO $\mathrm{B}$ inhibitors through the decoration of the $2 \mathrm{H}$-chromen-2one structural motif. Molecules 2016, 21, 362.

[47] Tonelli, M.; Catto, M.; Tasso, B.; Novelli, F.; Canu, C.; Iusco, G.; Pisani, L.; Stradis, A. D.; Denora, N.; Sparatore, A.; Boido, V.; Carotti, A.; Sparatore, F. Multitarget therapeutic leads for Alzheimer's disease: Quinolizidinyl derivatives of bi- and tricyclic systems as dual inhibitors for cholinesterases and $\beta$-amyloid (A $\beta$ ) aggregation. ChemMedChem 2015, 10, 1040-1053.

[48] Domínguez J. L.; Fernández-Nieto, F.; Castro, M.; Catto, M.; Paleo, M. R.; Porto, S.; Sardina, F. J.; Brea, J. M.; carotti, A.; Villaverde, M. C.; Sussman, F. 
Computer-aided structure-based design of multitarget leads for Alzheimer's disease. J. Chem. Inf. Model. 2015, 55, 135-148.

[49] Dias, K.S.T.; Viegas, C., Jr. Multi-target directed drugs: A modern approach for design of new drugs for the treatment of Alzheimer's disease. Curr. Neuropharmacol. 2014, 12, 239-255.

[50] Prati, F.; Uliassi, E.; Bolognesi, M. L. Two diseases, one approach: Multitarget drug discovery in Alzheimer's and neglected tropical diseases. MedChemComm 2014, 5, 853-861.

[51] Rostovtsev, V.V.; Green, L.G.; Fokin, V.V.; Sharpless, K.B. A stepwise Huisgen cycloaddition process: $\mathrm{Cu}(\mathrm{I})$-catalyzed regioselective "ligation" of azides and terminal alkynes. Angew. Chem. Int. Ed. 2002, 41, 2596-2599.

[52] Tornøe, C.W.; Christensen, C.; Meldal, M. J. Org. Chem. 2002, 67, 3057-3064.

[53] Struthers, H.; Mindt, T.L.; Schibli, R. Metal chelating systems synthesized using the copper(I) catalyzed azide-alkyne cycloaddition. Dalton Trans. 2010, 39, 675-696.

[54] Schulze, B.; Schubert, U.S. Beyond click chemistry - supramolecular interactions of 1,2,3-triazoles. Chem. Soc. Rev. 2014, 43, 2522-2571.

[55] Jiaranaikulwanitch, J.; Govitrapong, P.; Fokin, V.V.; Vajragupta, O. From BACE1 inhibitor to multifunctionality of tryptoline and tryptamine triazole derivatives for Alzheimer's disease, Molecules 2012, 17, 8312-8333.

[56] Lenhart, J.A.; Ling, X.; Gandhi, R.; Guo, T.L.; Gerk, P.M.; Brunzell, D.H.; Zhang, S. "Clicked" bivalent ligands containing curcumin and cholesterol as multifunctional $\mathrm{A} \beta$ oligomerization inhibitors: Design, synthesis, and biological characterization. J. Med. Chem. 2010, 53, 6198-6209.

[57] Ramprasad, J.; Nayak, N.; Dalimba, U.; Yogeeswari, P.; Sriram, D. One-pot synthesis of new triazole-imidazo[2,1-b][1,3,4]thiadiazole hybrids via click chemistry and evaluation of their antitubercular activity. Bioorg. Med. Chem. Lett. 2015, in press, DOI: 10.1016/j.bmcl.2015.08.009.

[58] Mishra, N.M.; Briers, Y.; Lamberigts, C.; Steenackers, H.; Robijns, S.; Landuyt, B.; Vanderleyden, J.; Schoofs, L.; Lavigne, R.; Luyten, W.; Van der Eycken, E.V. Evaluation of the antibacterial and anti-biofilm activities of novel CRAMPvancomycin conjugates with diverse linkers. Org. Biomol. Chem. 2015, 13, $7477-7486$. 
[59] Ma, L.-Y.; Pang, L.-P.; Wang, B.; Zhang, M.; Hu, B.; Xue, D.-Q.; Shao, K.-P.; Zhang, B.-L.; Liu, Y.; Zhang, E.; Liu, H.-M. Design and synthesis of novel 1,2,3-triazole-pyrimidine hybrids as potential anticancer agents, Eur. J. Med. Chem. 2014, 86, 368-380.

[60] Fabbrizzi, P.; Bianchini, F.; Menchi, G.; Raspanti, S.; Guarna, A.; Trabocchi, A. Combination of click chemistry and sulfonamides to develop three-armed triazole compounds, Tetrahedron 2014, 70, 5439-5449.

[61] Brabez, N.; Saunders, K.; Nguyen, K.L.; Jayasundera, T.; Weber, C.; Lynch, R.M.; Chassaing, G.; Lavielle, S.; Hruby, V.J. Multivalent interactions: Synthesis and evaluation of melanotropin multimers-tools for melanoma targeting. ACS Med. Chem. Lett. 2013, 4, 98-102.

[62] Liu, K.; Gandhi, R.; Chen, J.; Zhang, S. Bivalent ligands targeting multiple pathological factors involved in Alzheimer's disease. ACS Med. Chem. Lett. 2012, 3, 942-946.

[63] Howell, L.A.; Bowater, R.A.; O'Connell, M.A.; Reszka, A.P.; Neidle, S.; Searcey, M. Synthesis of small molecules targeting multiple DNA structures using click chemistry. ChemMedChem, 2012, 7, 792-804.

[64] Ouberai, M.; Brannstrom, K.; Vestling, M.; Olofsson, A.; Dumy, P.; Chierici, S.; Garcia, J. Clicked tacrine conjugates as acetylcholinesterase and $\beta$-amyloid directed compounds. Org. Biomol. Chem. 2011, 9, 1140-1147.

[65] Ronco, C.; Carletti, E.; Colletier, J.-P.; Weik, M.; Nachon, F.; Jean, L.; Renard, P.-Y. Huprine derivatives as sub-nanomolar human acetylcholinesterase inhibitors: From rational design to validation by X-ray crystallography. ChemMedChem 2012, 7, 400-405.

[66] Lewis, W.G.; Green, L.K.; Grynszpan, F.; Radić, Z.; Carlier, P.R.; Taylor, P.; Finn, M.G.; Sharpless, K.B. Click chemistry in situ: Acetylcholinesterase as a reaction vessel for the selective assembly of a femtomolar inhibitor from an array of building blocks. Angew. Chem. Int. Ed. 2002, 41, 1053-1057.

[67] Krasiński, A.; Radić, Z.; Manetsch, R.; Raushel, J.; Taylor, P.; Sharpless, K.B.; Kolb, H.C. In situ selection of lead compounds by click chemistry: Targetguided optimization of acetylcholinesterase inhibitors, J. Am. Chem. Soc. 2005, 127, 6686-6692.

[68] Whiting, M.; Muldoon, J.; Lin, Y.-C.; Silverman, S.M.; Lindstrom, V.; Olson, A.J.; Kolb, H.C.; Finn, M.G.; Sharpless, K.B.; Elder, J.H.; Fokin, V.V. 
Inhibitors of HIV-1 protease by using in situ click chemistry. Angew. Chem. Int . Ed. 2006, 45, 1435-1439.

[69] Sharpless, K.B; Manetsch, R. In situ click chemistry: A powerful means for lead discovery. Expert Opin. Drug Discovery 2006, 1, 525-538.

[70] Oueis, E.; Santoni, G.; Ronco, C.; Syzgantseva, O.; Tognetti, V.; Joubert, L.; Romieu, A.; Weik, M.; Jean, L.; Sabot, C.; Nachon, F., Renard, P.-Y. Reaction site-driven regioselective synthesis of AChE inhibitors. Org. Biomol. Chem. 2014, 12, 156-161.

[71] Kolb, H.C.; Sharpless, K.B. The growing impact of click chemistry on drug discovery. Drug Discovery Today 2003, 8, 1128-1137.

[72] LogP values estimated with www.molinspiration.com.

[73] Hein, J.E.; Fokin, V.V. Copper-catalyzed azide-alkyne cycloaddition (CuAAC) and beyond: new reactivity of copper(I) acetylides. Chem. Soc. Rev. 2010, 39, $1302-1315$.

[74] Díez-González, S. Well-defined copper(I) complexes for Click azide-alkyne cycloaddition reactions: one Click beyond. Catal. Sci. Technol. 2011, 1, $166-178$.

[75] Mendal, M.; Tornøe, C.W. Cu-catalyzed azide-alkyne cycloaddition. Chem. Rev. 2008, 108, 2952-3015.

[76] Bock, V.D.; Hiemstra, H.; van Maarseveen, J.H. Cu-catalyzed azide-alkyne “click" cycloadditions from a mechanistic and synthetic perspective. Eur. J. Org. Chem. 2006, 2006, 51-68.

[77] Shi, Z.-J.; Yu, D.-G. Cross-coupling reactions. Comprehensive Inorg. Chem. II 2013, 6, 47-77.

[78] Orsini, A.; Vitérisi, A.; Bodlenner, A.; Weibel, J.-M.; Pale, P. A chemoselective deprotection of trymethylsilyl acetylenes catalyzed by silver salts. Tetrahedron Lett. 2005, 46, 2259-2262.

[79] Hubálek, F.; Binda, C.; Khalil, A.; Li, M.; Mattevi, A.; Castagnoli, N.; Edmondson, D.E. Demonstration of isoleucine 199 as a structural determinant for the selective inhibition of human monoamine oxidase $\mathrm{B}$ by specific reversible inhibitors. J. Biol. Chem. 2005, 280, 15761-15766.

[80] Esteban, G.; Allan, J.; Samadi, A.; Mattevi, A.; Unzeta, M.; Marco-Contelles, J.; Binda, C.; Ramsay, R.R. Kinetic and structural analysis of the irreversible inhibition of human monoamine oxidases by ASS234, a multi-target compound 
designed for use in Alzheimer's disease. Biochim. Biophys. Acta 2014, 1844, 1104-1110.

[81] Pérez, V.; Marco, J. L.; Fernández-Alvarez, E.; Unzeta, M.; Relevance of benzyloxy group in 2-indolyl methylamines in the selective MAO-B inhibition. Br.J. Pharmacol. 1999, 127, 869-876.

[82] Di, L.; Kerns, E.H.; Fan, K.; McConnell, O.J.; Carter, G.T. High throughput artificial membrane permeability assay for blood-brain barrier. Eur. J. Med. Chem. 2003, 38, 223-232.

[83] Li, L.; Zhang, C.-W.; Ge, J.; Qian, L.; Chai, B.-H.; Zhu, Q.; Lee, J.-S.; Lim, K.L.; Yao, S. Q. A small-molecule probes for selective profiling and imaging of monoamino oxidase B activities in models of Parkinson's disease. Angew. Chem. Int. Ed. 2015, 54, 10821-10825.

[84] Friesner, R.A.; Murphy, R.B.; Repasky, M.P.; Frye, L.L.; Greenwood, J.R.; Halgren, T.A.; Sanschagrin, P.C.; Mainz, D.T. Extra precision Glide: Docking and scoring incorporating a model of hydrophobic enclosure for protein-ligand complexes. J. Med. Chem. 2006, 49, 6177-6196. 


\section{Bioorganic and Medicinal Chemistry}

CHECKLIST FOR COMPOUND CHARACTERIZATION only for new/unknown compounds jo- BMC

Corresponding Author

을

TO DISPLAY INSTRUCTIONS: Double-click HERE (Formula Bar must be visible: Select View>Formula Bar) TO CLOSE: Enter/Return

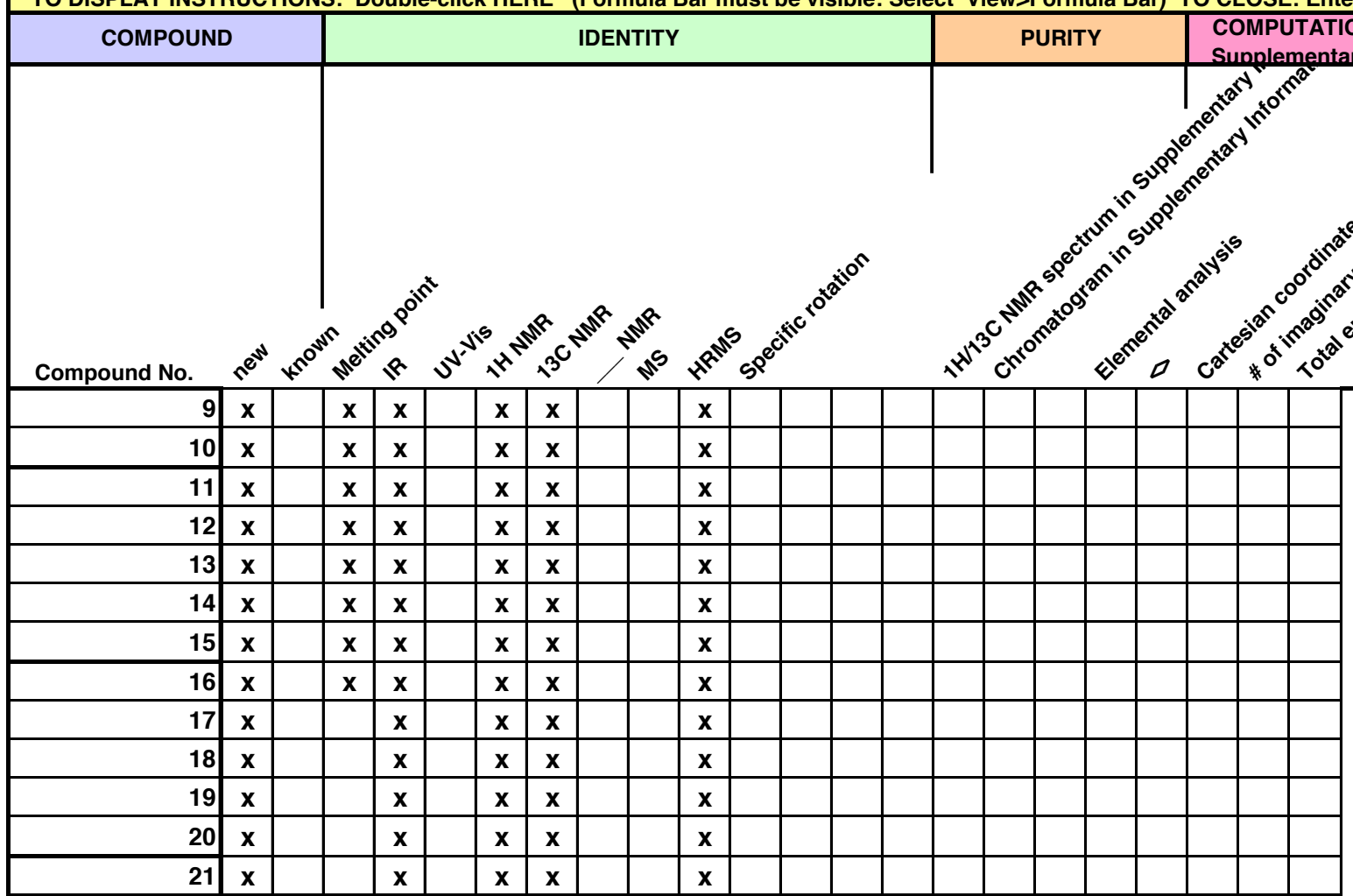

